\title{
Sensitivity Analysis of Gas Production from Class 2 and Class 3 Hydrate Deposits
}

\author{
Matthew T. Reagan, SPE, George J. Moridis, SPE, Keni Zhang, SPE, Lawrence Berkeley National Laboratory
}

\begin{abstract}
Gas hydrates are solid crystalline compounds in which gas molecules are lodged within the lattices of an ice-like crystalline solid. The vast quantities of hydrocarbon gases trapped in hydrate formations in the permafrost and in deep ocean sediments may constitute a new and promising energy source. Class 2 hydrate deposits are characterized by a Hydrate-Bearing Layer (HBL) that is underlain by a saturated zone of mobile water. Class 3 hydrate deposits are characterized by an isolated Hydrate-Bearing Layer (HBL) that is not in contact with any hydrate-free zone of mobile fluids. Both classes of deposits have been shown to be good candidates for exploitation in earlier studies of gas production via vertical well designs - in this study we extend the analysis to include systems with varying porosity, anisotropy, well spacing, and the presence of permeable boundaries. For Class 2 deposits, the results show that production rate and efficiency depend strongly on formation porosity, have a mild dependence on formation anisotropy, and that tighter well spacing produces gas at higher rates over shorter time periods. For Class 3 deposits, production rates and efficiency also depend significantly on formation porosity, are impacted negatively by anisotropy, and production rates may be larger, over longer times, for well configurations that use a greater well spacing. Finally, we performed preliminary calculations to assess a worst-case scenario for permeable system boundaries, and found that the efficiency of depressurization-based production strategies are compromised by migration of fluids from outside the system.
\end{abstract}

\section{Introduction}

Background. Gas hydrates are solid crystalline compounds in which gas molecules (referred to as guests) occupy the lattices of ice crystal structures (called hosts). Under suitable conditions of low temperature $T$ and high pressure $P$, the hydration reaction of a gas $\mathrm{G}$ is described by the general equation

$$
\mathrm{G}+N_{H} \mathrm{H}_{2} \mathrm{O}=\mathrm{G} \bullet N_{H} \mathrm{H}_{2} \mathrm{O} \text {, }
$$

where $N_{H}$ is the hydration number. Natural hydrates in geological systems usually contain hydrocarbons (mainly $\mathrm{CH}_{4}$ and other alkanes), but may also contain $\mathrm{CO}_{2}, \mathrm{H}_{2} \mathrm{~S}$ or $\mathrm{N}_{2}$. Hydrate deposits occur in two distinctly different geologic settings where the necessary conditions of low $T$ and high $P$ exist for their formation and stability: in the permafrost and in deep ocean sediments.

Although there has been no systematic effort to map and evaluate this resource and current estimates vary widely ${ }^{1,2,3}$ (ranging between $10^{15}$ to $10^{18} \mathrm{~m}^{3}$ ), the consensus is that the worldwide quantity of hydrocarbon gas hydrates is vast. Even the most conservative estimate exceeds the total energy content of the known conventional fossil fuel resources. The sheer magnitude of the resource, ever increasing global energy demand, and dwindling conventional fossil fuel reserves, point to hydrates a promising energy source ${ }^{4,5}$ even if only a limited number of deposits might be suitable for production and/or only a fraction of the trapped gas can be recovered. The attractiveness of hydrates is further enhanced by the environmental desirability of natural gas (as opposed to solid or liquid) fuels. The production potential of gas hydrate accumulations demands technical and economic evaluation.

Gas can be produced from hydrates by inducing dissociation, which also releases large amounts of $\mathrm{H}_{2} \mathrm{O}$ (Eq. 1). The three main methods of hydrate dissociation are ${ }^{6}$ : (1) depressurization, in which the pressure $P$ is lowered to a level lower than the hydration pressure $P_{e}$ at the prevailing temperature $T,(2)$ thermal stimulation, in which $T$ is raised above the hydration temperature $T_{e}$ at the prevailing $P$, and (3) the use of inhibitors (such as salts and alcohols), which shifts the $P_{e}-T_{e}$ equilibrium through competition with the hydrate for guest and host molecules.

Classification of hydrate deposits. Natural hydrate accumulations are divided into three main classes. ${ }^{7,8}$ Class 1 accumulations are composed of two layers: a hydrate-bearing layer (HBL) and an underlying two-phase fluid zone containing mobile gas and liquid water. The bottom of the hydrate stability zone (the location above which hydrates are stable) coincides with the bottom of the hydrate interval. Production from such deposits was discussed in detail by Moridis et al. ${ }^{8} \mathrm{Class} 2$ deposits consist of two zones: a HBL, overlying a zone of mobile water (WZ). Class 3 accumulations are composed of a single zone, the hydrate interval (HBL), and have no underlying zone of mobile fluids. In Classes 2 and 3, the entire HBL may be well within the hydrate stability zone and can exist under stable equilibrium conditions. Production from Class 2 and 
3 deposits via depressurization has studied in two papers ${ }^{9,10}$, and shown to be feasible and economical using existing technologies. An additional class, Class $4,{ }^{11}$ pertains specifically to oceanic accumulations, and involves disperse, lowsaturation hydrate deposits that lack confining geologic strata.

Objective and approach. The previous studies by Moridis and Reagan ${ }^{9,10}$ determined strategies for exploiting Class 2 and Class 3 deposits, proposing approaches that allow the extraction of large quantities of gas at high rates for long periods of time. The objective of this follow-up study is to quantify the effects of reservoir properties on this production potential. The previous research shows that depressurization-induced dissociation (based on fluid removal) is the most promising gas production strategy in both Classes of deposits because of its simplicity and its technical and economic effectiveness. With this in mind, we approach variations of the two Classes of deposits using the same production strategies, adjusting the approach, if necessary, to maintain efficient production from the system of interest. Our sensitivity studies examine 1) the effect of increased or decreased porosity, 2) the effect of anisotropy in formation permeability, and 3) the effect of increased or decreased well spacing. In addition, previous work has focused on gas production from accumulations that are confined between impermeable upper and lower boundaries (overburden and underburden, respectively). Hydrate accumulations without any such boundaries have been shown conclusively to have low gas production potential because of the limited effectiveness of depressurization, and produce large volumes of water. ${ }^{12}$ We must consider a third situation-formations upper and lower boundaries with low permeability, but a permeability significantly less than that of the deposit itself (for example, permeable shales). To quantify this effect for Class 2 and Class 3 deposits, we study two cases with lowpermeability confining layers.

\section{Geological System Description and \\ Numerical Representation}

The reference geologic system. The geologic system in this study is again based the Tigershark area ${ }^{13}$, located in the Alaminos Canyon Block 818 of the Gulf of Mexico (Figure 1). Data from an exploration well in $2750 \mathrm{~m}(9000 \mathrm{ft}$ ) of water indicated the presence of an $18.25-\mathrm{m}(60-\mathrm{ft})$ thick, sandy hydrate zone (10,530 to $10,590 \mathrm{ft}$ drilling depth) with a porosity $\phi$ of about 0.30 and Darcy-range intrinsic permeability $k$. Resistivity and p-wave velocity data ${ }^{14}$ indicate a gas hydrate saturation, $S_{H}$, from 0.6-0.8. Preliminary calculations indicated that the base of the gas hydrate stability zone occurs at, or slightly below the base of the HBL. ${ }^{13,14}$ The Tigershark data are particularly valuable because they describe a promising target for gas production, as indicated by the high $S_{H}$ and the thermodynamic proximity of the HBL to the bottom of the hydrate stability zone.

Model description: The simulated deposit has the properties and initial conditions of the Tigershark reference geologic system described above, with boundary conditions created for the study, matching the system simulated previously. ${ }^{9,10}$

The Class 2 system initially has an impermeable upper boundary, a 15-m thick WZ underneath the HBL, and an impermeable lower boundary (Figure 2). While a low-permeability upper boundary can be deduced from the well log data in Figure 2, there was insufficient information to establish the presence of a lower boundary. The initial thickness of both the overburden and the underburden (the heat-exchanging boundaries) is $30 \mathrm{~m}$, which earlier calculations $\mathrm{s}^{8,15}$ had indicated to be sufficient to allow accurate heat exchange with the hydrate deposit during a 30-yr long production period. The Class 3 deposit has the properties and initial conditions of the Class 2 system (Table 1), but the WZ is removed and replaced with an additional $15 \mathrm{~m}$ of underburden (see Figure 3).

A cylindrical hydrate deposit is assumed (Figures 2 and 3), with a well at its center. The inner radius of the system is the well radius $r_{w}=0.1 \mathrm{~m}$ and its outer radius, a no-flow boundary, is initially placed at $r_{\max }=800 \mathrm{~m}$. This corresponds to a well spacing of about 200 ha ( $=500$ acres), with the no-flow boundary at $r_{\max }$ representing the effect of other wells present in a regular spacing pattern.

The hydraulic and thermal properties of the various formations in the domain under investigation are listed in Table 1. The relative permeability relationship and the corresponding parameters are based on data from the first field test of gas production from hydrates at the Mallik site ${ }^{16}$, while the capillary pressure relationships and parameters are consistent with the texture, porosity and permeability of the Tigershark formation.

The numerical simulation code. The numerical studies in this paper were conducted using the TOUGH+HYDRATE simulator, ${ }^{17}$ the successor to the earlier TOUGH-Fx/HYDRATE model and a descendent of the TOUGH2 family of codes. This code can model the non-isothermal hydration reaction, phase behavior, and flow of fluids and heat under conditions typical of natural $\mathrm{CH}_{4}$-hydrate deposits in complex geologic media. It includes both an equilibrium and a kinetic model ${ }^{18,19}$ of hydrate formation and dissociation. The model accounts for heat and up to four mass components (i.e., water, $\mathrm{CH}_{4}$, hydrate, and water-soluble inhibitors such as salts or alcohols) that are partitioned among four possible phases: gas, aqueous liquid, ice, and hydrate. A total of 15 states (phase combinations) can be described by the code, which can handle any combination of hydrate dissociation mechanisms and can describe the phase changes and steep solution surfaces that are typical of hydrate problems.

\section{Simulation specifics}


Domain discretization. The cylindrical domain is discretized into 119 × $113=13,447$ gridblocks in $(r, z)$. The uppermost and lowermost layers correspond to infinite, constant temperature, no-flow boundaries, while the layers corresponding to the top and bottom confining layers (Table 1) are initially impermeable but allow heat exchange between the deposit and the surrounding formations. To describe accurately the processes and phenomena occurring in the vicinity of the wellbore, known to be critically important to production, we use a very fine discretization in the $r$ dimension with the first $20 \mathrm{~m}$.

The HBL was subdivided into vertical segments of uniform $\Delta z=0.25 \mathrm{~m}$. As demonstrated in Moridis et al. ${ }^{8}$ and Moridis and Reagan ${ }^{9,10}$, such a fine discretization of the hydrate zone is necessary for accurate predictions of hydrate dissociation and description of near-well processes. Conditions in the WZ allowed a coarser discretization, involving vertical subdivisions of $\Delta z=0.25 \mathrm{~m}, 0.5 \mathrm{~m}$, and $1 \mathrm{~m}$. This fine discretization provided a high level of detail near the wellbore and in the entire hydrate-bearing zone. Assuming equilibrium hydrate dissociation, ${ }^{20}$ this grid resulted in 53,788 coupled equations that were solved simultaneously.

Initial conditions. We determined the initial conditions in the reservoir by following the initialization process described by Moridis et al. ${ }^{8}$ Pressure distributions in the oceanic subsurface were assumed to follow a hydrostatic distribution, consistent with observations of natural hydrate accumulations in geologic media. ${ }^{21}$ The initial pressures were computed using the ocean water depth and the HBL elevation, in addition to a pressure-adjusted saline water density typical of ocean water $\left(1030 \mathrm{~kg} / \mathrm{m}^{3}\right.$ at atmospheric pressure). Because the bottom of the hydrate stability zone was known to occur at or slightly below the known elevation of the HBL base (which corresponded to a known hydrostatic pressure), the hydrate base temperature $T_{B}$ was selected to be slightly lower than the equilibrium hydration temperature $T_{e}$ at that location (see Table 1, and Figure 4). Using $T_{B}=5{ }^{\circ} \mathrm{C}$, the known temperature at the mud line, and the corresponding elevations, the local geothermal gradient was computed as $d T / d z=0.03464 \mathrm{~K} / \mathrm{m}$ and this was used to estimate the initial temperature distribution in the domain.

Well description. The numerical simulations included a physical representation of the wellbore. The physics of multiphase flow in a wellbore are very different from the physics of multiphase flow and transport in porous media. A physically rigorous description would involve solving the complete Navier-Stokes equation. However, an analysis of this possibility has indicated that the resulting system would require very small timesteps and very long execution times. We use an alternative approach that involves an approximation of the Navier-Stokes solution of wellbore flow by the process of Darcy flow through a pseudo-porous medium representing the interior of the well. This pseudo-porous medium has a porosity of 1.0 , high permeability $\left(10^{-9} \mathrm{~m}^{2}=1,000\right.$ Darcies $)$, zero capillary pressure, and a relative permeability that is a linear function of the phase saturations in the wellbore. The use of a small irreducible gas saturation in the fictitious wellbore porous medium (usually $0.5 \%$ ) is necessary to allow the emergence of a free gas phase in the well. The desired total mass production rate, or a fixed wellhead pressure, is then applied to a single gridblock located at the top of the subdomain that describes the wellbore. Our analyses have shown that the use of this approximation results in a solution that deviates by less than $5 \%$ from the more accurate solution of the Navier-Stokes equation, while reducing the execution time by a factor of at least 2 .

Simulation process and outputs. The simulation period was up to 30 years, corresponding to the typical life span of a well, but the deposit was often exhausted much earlier, or in some cases abandoned due to insufficient production. In the course of the simulation, we monitored: 1) spatial distributions of (a) temperature, (b) gas and hydrate phase saturations, and (c) water salinity; 2) Volumetric rate of $\mathrm{CH}_{4}$ produced at the well $\left(Q_{P}\right)$; 3) Cumulative volume of $\mathrm{CH}_{4}$ produced at the well $\left(V_{P}\right)$; and 4) cumulative mass of water produced at the well $\left(M_{W}\right)$. We also compute two ratios to compare production efficiency between cases: 1) the cumulative average production rate at time $t\left(Q_{\text {avg }}\right)$ and 2$)$ the ratio of cumulative water production to cumulative volume of $\mathrm{CH}_{4}$ produced $\left(R_{W G C}=M_{W} / V_{P}\right)$.

Class 2 Production: variable production interval with wellbore heating. Optimized Class 2 production ${ }^{10}$ involved a well with the perforated interval initially extending $5 \mathrm{~m}$ into the $\mathrm{WZ}$, with heating along the outer surface of the wellbore $\left(Q_{\Theta}=\right.$ $300 \mathrm{~W} / \mathrm{m})$, and an initial production rate, $Q_{M}$, of $18.955 \mathrm{~kg} / \mathrm{s}(10,000 \mathrm{BPD})$. This heating causes localized thermal dissociation of hydrate and the creation of a cylindrical dissociated region around the well, allowing communication between the production interval and the upper boundary of the hydrate zone. Electrical heating is used initially (as the $S_{H}$ of the undisturbed HBL is sufficiently high to make flow difficult), and warm water injection (injected at the upper part of the wellbore) is used at a later times (at a rate $Q_{I}=1 \mathrm{~kg} / \mathrm{s}$ at an enthalpy of $H_{W}=2.75 \times 10^{5} \mathrm{~J} / \mathrm{kg}$ ). This replaces slow and inefficient heat conduction with the much faster and efficient advective heat transfer mechanism. ${ }^{10}$ This well configuration prevents the formation of secondary hydrate around the wellbore, maintains a channel between the production interval and all dissociating interfaces, and eliminates potential blockage of flow to the production interval. As the hydrate interface recedes during dissociation, it is possible to expand the production interval upward into the HBL. At a later stage (usually when less than $35 \%$ of the original hydrate remains), when there is significant gas accumulation at the top of the reservoir, the enhanced-recovery method of Moridis ${ }^{10}$ may be used to access this gas without risk of large-scale blockages by secondary hydrate or ice formation. Our sensitivity cases follow this production strategy, with the timing of production strategy changes and production rate adjustments selected to match production conditions and evolution for each case.

Class 3 Production: Constant-P depressurization. The Class 3 reference case ${ }^{9}$ involved a simple well design that employed a constant bottomhole pressure described by an internal boundary located in a gridblock above the uppermost gridblock in the well subdomain. By imposing a constant bottomhole pressure $P_{w}$ and a realistic constant temperature $T_{w}$ at 
this boundary, the correct constant- $P$ condition was applied to the well while avoiding any non-physical temperature distributions in the well itself. Note that the initial bottomhole pressure, $P_{w}=3 \mathrm{MPa}$, is greater than $P_{Q}$, eliminating the possibility of ice formation and the corresponding deleterious effect on $k_{\text {eff. }}$. At a later stage of production, $P_{w}$ was safely reduced to $P_{w}=0.5 \mathrm{MPa}<P_{Q}$ to maximize $Q_{P}$ and $V_{P}$, without the possibility of ice formation. Because of the well configuration used in Class 3 production, secondary hydrate can form next to the wellbore due to cooling from the endothermic hydrate dissociation reaction, as well as the Joule-Thompson phenomenon (which is at its maximum in the immediate vicinity of the well where the highest gas flow velocities occur). The previous study showed that these secondary hydrate blockages form and dissociate over time, creating large variations in production rate, but they are "self-clearing" over longer times and production recovers automatically. ${ }^{9}$ This production method is based on a very simple design, involves only conventional technology, and poses no particular technical challenges. Our sensitivity cases use this method for Class 3 production.

\section{Parametric Sensitivity During Production from Class 2 Deposits}

As the description of the reference case is provided in great detail in Moridis and Reagan ${ }^{10}$, we aim here to describe the important differences between the reference case and selected parametric variations. We investigated the sensitivity of gas production and the evolution of the reservoir to the following conditions and parameters:

(a) The porosity of the underlying porous medium,

(b) The degree of anisotropy in the permeability of the porous medium,

(c) The well spacing.

For each variation, all other simulation parameters are maintained at the conditions of the reference case.

\section{Sensitivity variation (a): Porosity}

To assess the effect of the porosity of the underlying porous medium, we varied the porosity of the WBL and WZ from the reference value of $\phi=0.30$ to $\phi=0.20$ and $\phi=0.40$, maintaining other porous-media properties at reference values.

Evolution of $\boldsymbol{Q}_{P}, \boldsymbol{V}_{\boldsymbol{P}}, \boldsymbol{Q}_{\text {avg }}$, and $\boldsymbol{R}_{W G C}$. Figures 6 and 7 compare the Class 2 reference case (gray lines) ${ }^{10}$ to decreased and increased porosity for the first 3,500 days of production. Reducing the porosity from the reference value of $\phi=0.30$ to $\phi=$ 0.20 (blue line) shifts the $Q_{P}$ vs. time curve (Figure 6) to the left, with production increasing more rapidly and the first production peak, just before the first cavitation, occurring approximately 400 days earlier and reaching $3.7 \mathrm{ST} \mathrm{m} / \mathrm{s}(11.2$ MMSCFD). Increasing the porosity to $\phi=0.40$ (red line) shifts the production curve to the right, with the first cavitation occurring about 530 days later than in the reference case, with production peaking at $2.1 \mathrm{ST} \mathrm{m}^{3} / \mathrm{s}$ (6.4 MMSCFD). Several effects contribute to these variations. First, a decreased porosity reduces the pore space available for hydrate in the formation, decreasing the total mass of hydrate but also reducing the overall volume available for fluid transport. However, the increase in the mass of rock per unit volume of pore space increases the latent heat available to the dissociating hydrate during depressurization. As a result, we see a more rapid onset of gas production and higher peak production during Stages I and II ${ }^{10}$ (initiation and increasing production) of the production strategy. Conversely, increased permeability results in greater total pore volume and a larger mass of hydrate, but the porous medium provides correspondingly less latent heat for dissociation. A larger mass of hydrate to be dissociated, combined with less latent heat immediately available during dissociation, slows the initiation and evolution of gas production in Stages I and II.

Cumulative production of methane (Figure 7) reflects the consequences of these effects. For the low-porosity case, cumulative $\mathrm{CH}_{4}$ produced $\left(V_{P}\right)$ exceeds that of the reference case within the first 2,700 days of production. The ratio of total water mass removed per $\mathrm{m}^{3}$ of gas produced, $R_{W G C}$, quickly decreases below $R_{W G C}$ for the reference case. For the highporosity case, $V_{P}$ develops more slowly, and the water-to-gas ratio is consistently higher after $t=500$ days. However, close examination of $V_{P}$ evolution also shows that the cumulative gas produced at the time of first cavitation is similar for all three cases ( $\sim 1 \times 10^{8} \mathrm{ST} \mathrm{m}^{3}$ or $\left.\sim 3,500 \mathrm{MMSCF}\right)$, while $R_{W G C}$ decreases slightly with decreasing porosity.

For each case, a 5-day injection of heated seawater $\left(Q_{I}=1 \mathrm{~kg} / \mathrm{s}\right.$ at $\left.H_{W}=2.75 \times 10^{5} \mathrm{~J} / \mathrm{kg}\right)$ was used to clear the secondary hydrate blockage, with production resuming at a reduced rate $Q_{M}=16.11 \mathrm{~kg} / \mathrm{s}$. During this stage of production, TOUGH+HYDRATE automatically cuts the production rate by $2 \%$ each time cavitation occurs $\left(P_{W}<0.2 \mathrm{MPa}\right)$, allowing the simulation to continue automatically. Production resumes at a decreased rate for both cases, but rapidly recovers. For the low-porosity case, production peaks near 1,700 days at $5.8 \mathrm{ST} \mathrm{m}^{3} / \mathrm{s}$ (17.7 MMSCFD) before automated rate-cutting proceeds. A second cavitation is not seen for the high-porosity case within the observed 3,500-day period.

Spatial distributions of $\boldsymbol{S}_{\boldsymbol{H}}, \boldsymbol{S}_{G}, \boldsymbol{T}$, and $\boldsymbol{X}_{\boldsymbol{i}}$. The evolution of the deposit over the course of production can be seen in Figures 8 through 11.

Figure 8 compares the 2-D distribution of hydrate saturation, $S_{H}$, for $\phi=0.20$ (left column) and $\phi=0.40$ (right column) at selected times throughout the simulated period. Figures $8 \mathrm{a}$ and $8 \mathrm{~b}$ each show hydrate saturation at the time of the first cavitation. In both cases, we see secondary hydrate formation near the production interval $(\mathrm{z} \sim-48 \mathrm{~m})$. For the low-porosity case (panel a), we see increased degree of hydrate dissociation (lower $S_{H}$ ) within the hydrate mass although less time has passed, and we also note accumulation of secondary hydrate at the inner and lower boundaries of the hydrate zone. The highporosity case (panel b) has a greater $S_{H}$ at the time of first cavitation, although the spatial extent of the remaining hydrate is quite similar. The corresponding distributions of gas saturation, $S_{G}$, are shown in Figures $9 \mathrm{a}$ and $9 \mathrm{~b}$. For both cases, 
significant gas has accumulated at the top of the deposit, and the expected upper dissociation interface has formed below the overburden. This gas bubble is slightly larger in extent in the low-porosity case (mainly due to greater recession at the top of the deposit) and the gas, migrating through the bulk of the hydrate mass, forms secondary hydrate at the inner radius of the receding hydrate. Figures $10 \mathrm{a}$ and $10 \mathrm{~b}$ show corresponding temperature distributions. The low-porosity case exhibits higher temperatures within the remaining hydrate mass, despite greater dissociation (an endothermic process). Figures 11a and 11b display inhibitor $(\mathrm{NaCl})$ mass fraction in the aqueous phase. The low-porosity case, by virtue of a greater degree of dissociation, shows greater freshening of the aqueous phase within the hydrate mass itself and in the water migrating downward through the deposit.

The next sets of panels show the properties of the system immediately after the $5 \mathrm{~d}$ injection period. Figures $8 \mathrm{c}$ and $8 \mathrm{~d}$ clearly show that all secondary hydrate next to the well has been removed, and Figures $9 \mathrm{c} / 9 \mathrm{~d}, 10 \mathrm{c} / 10 \mathrm{~d}$, and $11 \mathrm{c} / 11 \mathrm{~d}$ all show the extent of the injection plume in terms of displaced gas phase, increased temperature, and inhibitor mass fraction, respectively. Panels e and $\mathrm{f}$ in Figure 8-11 track the further evolution of the system as production continues after resumption at a lower mass production rate, including the reformation of the region of free gas at the top of the deposit (Figure 9e/f), additional cooling (Figure 10e/f), and significant reductions in the salinity of the aqueous phase (Figure 11e/f).

\section{Sensitivity variation (b): anisotropy.}

To simulate variations in the vertical permeability of the system due to layered structures within the underlying porous media, the vertical permeability, $k_{z}$, was reduced by a factor of $10\left(k_{z} / k_{r}=0.10\right)$ and a factor of $20\left(k_{z} / k_{r}=0.05\right)$ while maintaining the horizontal permeability at the reference value.

Evolution of $\boldsymbol{Q}_{P}, \boldsymbol{V}_{\boldsymbol{P}}, \boldsymbol{Q}_{\text {avg, }}$, and $\boldsymbol{R}_{W G C}$. Evolution of $Q_{P}$ and $Q_{a v g}$ for all three cases is seen in Figure 12. For both anisotropic cases, the evolution of $Q_{P}$ from initiation of production to the point of first cavitation is similar to the reference case, with the time of first cavitation delayed by slightly more than 100 days for $k_{z} / k_{r}=0.10$ and approximately 250 days for the $k_{z} / k_{r}=0.05$ case. Peak production was nearly identical for all three cases. After five days of hot water injection, production was resumed with a larger production interval and warm ocean water circulation at the top of the wellbore. After cleanup, both cases produce gas at significantly lower rates than in the reference case for the next 2,000 days of production. For $k_{z} / k_{r}=0.10$, production increases for 1,200 days, peaking at $5.1 \mathrm{ST} \mathrm{m} / \mathrm{s}$ (15.6 MMSCFD) before cuts in mass production are required. Mass-rate cutting continues until 5,800 days, at which point the system pressure has fallen below the quadruple point and a fully open wellbore ( $t>5,900$ days) can be used to extract the remaining gas. The system is exhausted at 7,000 days. For the $k_{z} / k_{r}=0.05$ case, however, production increases more slowly after cleanup, reaching a peak of $4.1 \mathrm{ST} \mathrm{m} / \mathrm{s}(12.5 \mathrm{MMSCFD})$ at 2,900 days after the resumption of production. However, after this peak the system is unable to reduce the mass production rate gradually; instead, substantial cuts in mass production, forced by low pressures in the wellbore, drive the production rate below 2.0 ST m $\mathrm{S}^{3} / \mathrm{s}$ (6.1 MMSCFD), and increasing production is not seen until 7,400 days, at which point the formation pressure has dropped low enough to allow open-well production and the removal of the remaining gas. The evolution of average production rates, $Q_{a v g}$, tracks the rates themselves, as expected.

Figure 13 shows the evolution of cumulative gas production, $V_{P}$, and water-to-gas ratio, $R_{W G C}$ for all three cases. A total of over $1.4 \times 10^{9} \mathrm{ST} \mathrm{m}^{3}$ of $\mathrm{CH}_{4}$ is produced for both cases, although as expected from the rates in Figure 12, greater anisotropy results in slower evolution of total production. $R_{W G C}$ for both anisotropic cases evolve similarly up to the first cavitation, after which time the $k_{z} / k_{r}=0.05$ case produces more water per $\mathrm{m}^{3}$ of $\mathrm{CH}_{4}$ produced but achieves roughly the same ratios as the less anisotropic cases at later times.

Spatial distributions of $S_{H}, S_{G}, T$, and $X_{i}$. The evolution of the deposit over the course of production can be seen in Figures 14 through 17.

Figures $14 \mathrm{a}$ and $14 \mathrm{~b}$ show the hydrate saturation. $S_{H}$, for $k_{z} / k_{r}=0.10$ (left column) and $k_{z} / k_{r}=0.05$ (right column) cases, respectively, at the time of the first cavitation. Although Figure $14 \mathrm{~b}$ shows that the $k_{z} / k_{r}=0.05$ case has developed more secondary hydrate near the production interval, the systems look quite similar at this stage. Gas saturations, showing in Figures 15a and 15b, have evolved in parallel as well. In Figures 16a and 16b, a greater drop in temperature due to JouleThompson effects (steeper pressure gradients/higher gas velocities) is seen adjacent to the production interval for $k_{z} / k_{r}=0.05$ behind the more extensive secondary hydrate barrier. Inhibitor mass fractions, seen in Figures 17a and 17b, are similar for both cases. As production continues, differences in the evolution of the system become more apparent. In Figures $14 \mathrm{c}$ and $14 \mathrm{~d}$, the shape of the remaining hydrate mass is clearly more elongated for the case of greater anisotropy. Figure $15 \mathrm{c}$ and $15 \mathrm{~d}$ show lower gas saturations above the hydrate for $k_{z} / k_{r}=0.05$, and also show that the injected ocean water (region of low $S_{G}$ near the upper left) extends twice as far, radially, for the higher-anisotropy case. The temperature profiles in Figure 16c and $16 \mathrm{~d}$ confirm this, with the region of substantial heating extending further from the injection point for the $k_{z} / k_{r}=0.05$ case. In Figure $17 \mathrm{c}$ vs. Figure 17d, we can see the horizontal progress of the injected seawater water as an extended region of high salinity. Panels e and $\mathrm{f}$ in Figures 14-17 show conditions at the peak of production, and the effects of anisotropy, biasing the system toward horizontal transport, are apparent in each case. Panels $g$ and $h$ in Figures 14-17 show conditions after solid hydrate is depleted. Figures $15 \mathrm{~g}$ and $15 \mathrm{~h}$ show that the remaining "gas bubble" along the overburden is pushed outward, radially, in the $k_{z} / k_{r}=0.05$ case in comparison to the low-anisotropy case. Temperature profiles in Figures $16 \mathrm{~g}$ and $16 \mathrm{~h}$ and inhibitor distribution in Figures $17 \mathrm{~g}$ and $17 \mathrm{~h}$ reveal that low-salinity fluids produced from hydrate dissociation are moving radially inward on a path aligned with the level of the production interval, with vertical transport and mixing inhibited by reduced vertical permeability. Figure $16 \mathrm{~h}$ also shows warmer temperatures near the wellbore as a result of reduced transport 
of cold gas and water from the region of hydrate dissociation (constrained by the reduced vertical permeability), and Figure $17 \mathrm{~h}$ confirms this, showing only a narrowed zone of freshened water approaching the wellbore.

\section{Sensitivity variation (c): well spacing.}

The Class 2 reference case ${ }^{10}$ used a well spacing of 200 ha (500 acres). To gauge sensitivity to well spacing, two new meshes were constructed, using the same discretization applied to maximum radii of $562.2 \mathrm{~m}(a=100 \mathrm{ha})$ and $1128.4 \mathrm{~m}(a=$ $400 \mathrm{ha})$.

Evolution of $\boldsymbol{Q}_{P}, \boldsymbol{V}_{\boldsymbol{P}}, \boldsymbol{Q}_{\text {avg }}$, and $\boldsymbol{R}_{W G C}$. The effect of well spacing on production rate is clearly seen in Figure 18 . With a reduced well spacing of $100 \mathrm{ha}$, production (and cumulative average production) quickly accelerates beyond that seen in the reference case, peaking at $4.0 \mathrm{ST} \mathrm{m}^{3} / \mathrm{s}(12.2 \mathrm{MMSCFD})$ at 1,017 days, at which point we experience the first cavitation. Production for the 400 ha case, in contrast, develops more slowly, not reaching the first cavitation until 3,299 days at a rate of $2.65 \mathrm{ST} \mathrm{m}^{3} / \mathrm{s}(8.1 \mathrm{MMSCFD})$. This is consistent with the use of a single mass production rate to depressurize (and initiate hydrate dissociation) in reservoirs of drastically different extents. All cases receive 5 days of warm ocean water injection to clear secondary hydrate obstructions, and then production is resumed. The 100 ha case reaches peak production at 1,700 days at a rate of $5.7 \mathrm{ST} \mathrm{m}^{3} / \mathrm{s}(17.4 \mathrm{MMSCFD})$, which is slightly less than the peak production rate for the reference case, ${ }^{10}$ of $6 \mathrm{ST}$ $\mathrm{m}^{3} / \mathrm{s}$ (although achieved 1,200 days earlier). The 400 ha case peaks after 5,500 days at a rate of $5.9 \mathrm{ST} \mathrm{\textrm {m } ^ { 3 }} / \mathrm{s}(18.1$ MMSCFD). Cumulative production, $V_{P}$, for all cases is shown in Figure 19, with tighter well spacing producing a larger quantity gas in less time. Water-to-gas ratios also favor tight well spacing. Unlike the previous Class 2 cases, total production at the time of first cavitation is significantly larger for the 400 ha case $\left(2.15 \times 10^{8} \mathrm{ST} \mathrm{m}^{3}\right)$ than for the 100 ha case $\left(1 \times 10^{8} \mathrm{ST}\right.$ $\left.\mathrm{m}^{3}\right)$.

Spatial distributions of $S_{H}, S_{G}, T$, and $X_{i}$. The evolution of the deposit over the course of production can be seen in Figures 20 through 23.

Figures 20a (100 ha, left column) and 20b (400 ha, right column) show the configuration of the remaining hydrate at the point of the first cavitation. As suggested by the cumulative production curves, more hydrate has been dissociated at this point for a system using wider well spacing. The extent of the gas zone is similar for both cases (Figures 21a and 21b), but temperature profiles (Figures 22a and 22b) indicate a substantially colder system at the point of cavitation for tighter spacing despite a much greater time for system evolution, and also lower salt mass fractions in the aqueous phase (Figures 23a and 23b). Panels $\mathrm{c}$ and $\mathrm{d}$ in all four figures show the state system after warm ocean water injection and the removal of secondary hydrate, and panels e and $\mathrm{f}$ show the state of the system during the following stage of increasing production. In Figures $21 \mathrm{e}$ and $21 \mathrm{f}$, the more tightly spaced system exhibits a more extensive bubble of free gas above the remaining hydrate (and greater recession of the hydrate mass from the overburden) with higher gas saturations in the region near the wellbore. Figures $23 \mathrm{e}$ and $23 \mathrm{f}$ show greater freshening of the aqueous phase (due to water released during dissociation) for the 100 ha example.

\section{Parametric Sensitivity During Production from Class 3 Deposits}

Once again, the reference case has been described in great detail elsewhere. ${ }^{9}$ We investigated the sensitivity of gas production and evolution of the reservoir to the following conditions and parameters:

(d) The porosity of the underlying porous medium,

(e) The degree of anisotropy in the permeability of the porous medium,

(f) The well spacing.

For each variation, all other simulation parameters are maintained at the conditions of the reference case, and use the Class 3 production strategy of constant- $P$ depressurization.

\section{Sensitivity variation (d): porosity}

Evolution of $\boldsymbol{Q}_{\boldsymbol{P}}, \boldsymbol{V}_{\boldsymbol{P}}, \boldsymbol{Q}_{\text {avg }}$, and $\boldsymbol{R}_{W G C}$. The evolution of $Q_{P}$ vs. time is presented in Figure 24. As seen in the reference case, production from Class 3 deposits is a cyclical process, with alternating periods of increasing production, cavitation, and recovery. As in the Class 2 case, the porosity was varied from the reference value of $\phi=0.30$ to $\phi=0.20$ and 0.40 . Decreasing the porosity, as before, shifts $Q_{P}$ evolution to shorter times, with the increased available latent heat assisting hydrate decomposition. The first cavitation and recovery occurs 230 days earlier than in the reference case, with a peak production rate of $4.5 \mathrm{ST} \mathrm{m}^{3} / \mathrm{s}$ (13.7 MMSCFD) and recovery at $2.0 \mathrm{ST} \mathrm{m}^{3} / \mathrm{s}(6.1 \mathrm{MMSCFD})$. The higher-porosity case, as expected, exhibits a delay in the development of production rate, with the first peak occurring 110 days later at a rate slightly below that of the reference case, $3.8 \mathrm{ST} \mathrm{m} / \mathrm{s}$ (11.6 MMSCFD), recovering to $1.8 \mathrm{ST} \mathrm{m}^{3} / \mathrm{s}$ (5.5 MMSCFD). Production in all cases increases after the first cavitation, with the low-porosity case exhibiting significantly higher rates through all simulation times, as reflected in the evolving average production rates (dotted lines). For the low-porosity case, a second cavitation and recovery is observed at $t=780$ days, with $Q_{P}$ reaching $7.6 \mathrm{ST} \mathrm{m}^{3} / \mathrm{s}(23 \mathrm{MMSCFD})$ and recovering at $1.6 \mathrm{ST} \mathrm{m} / \mathrm{s}(4.9$ MMSCFD).

Cumulative production, $V_{P}$, seen in Figure 25, consequently shows a strong trend of increased total production at all time as a function of reduced porosity. Water-to-gas ratios also decrease with decreasing porosity.

Spatial distributions of $\boldsymbol{S}_{\boldsymbol{H}}, \boldsymbol{S}_{\mathrm{G}}, \boldsymbol{T}$, and $\boldsymbol{X}_{\boldsymbol{i}}$. Figures 26 through 29 show the evolution of system properties over the course of production. 
Figures 26a and 26b show hydrate saturation, $S_{H}$, just before the point of first cavitation for both low (left column) and high (right column) porosities. As seen in previous studies of Class 3 production, ${ }^{9}$ the cycles of production and cavitation are driven by the formation and dissolution of a series of moving secondary hydrate barriers. The formation of the first barrier begins at the top of the wellbore and extends downward until production is blocked. The barrier then moves away from the wellbore (Figures 26c/d), as the inner radius is subjected to strong depressurization while the outer radius can accumulate new secondary hydrate as this zone is subject to strong Joule-Thompson effects. In Figure 26c, we also see a second barrier forming in the low-porosity case, anticipating the second cavitation noted at $t=780$ days in Figure 24. Figure 27a and 27b show $S_{G}$ at the point of first cavitation. In both cases, free gas is accumulating behind the forming secondary hydrate barrier, restricting the availability of gas at the well. In Figure 27c, the second barrier has also entrained gas between the obstructions, as well as a large region of gas behind the outer barrier. The high-porosity case (Figure 27d) has accumulated a smaller bubble of free gas at 927 days, and has not yet formed a second moving barrier. Figure 28 compares the evolution of temperature for both porosities. In Figure 28a, we find the lowest temperatures near the upper wellbore, where in Figure 26a a secondary hydrate barrier is forming; while in Figure 28b the completed barrier focuses the coldest temperatures (due to steep pressure gradients around the through the blockage) inside the barrier. As the first barrier moves away from the wellbore in either case, we see in Figures 28c/d that the lowest temperatures are found inside the inner barrier; in the lowporosity case the low temperatures are confined to the narrow region inside the recently formed barrier, while in the highporosity case distributed inside the larger region encompassed by the outer barrier. Distribution of inhibitor mass fraction (Figure 29), indicates greater freshening of the aqueous phase in a shorter period of time for the low-porosity case (Figures $29 \mathrm{a}$ and $29 \mathrm{c}$ ) vs. the high-porosity case (Figures $29 \mathrm{~b}$ and 29d). Inhibitor distribution also reveals the identity of the "active" moving barrier (in Figures 29c and 29d), as formation of hydrate excludes salt resulting in localized salinity increases at the advancing edge of the obstruction.

\section{Sensitivity variation (e): anisotropy.}

To simulate variations in the vertical permeability of the system due to layered structures within the underlying porous media, the vertical permeability, $k_{z}$, was reduced by a factor of $10\left(k_{z} / k_{r}=0.10\right)$ and a factor of $20\left(k_{z} / k_{r}=0.05\right)$.

Evolution of $Q_{P}, V_{P}, Q_{a v g}$, and $\boldsymbol{R}_{W G C}$. Figure 30 plots the evolution of production, $Q_{P}$, and average production vs. time for all three cases. Reduced permeability in the vertical direction shifts the first cavitation event later in time, and results in higher production rates up to the point of cavitation. For $k_{z} / k_{r}=0.10$, the first cavitation occurs at 590 days at a peak production rate of $6.25 \mathrm{ST} \mathrm{m} / \mathrm{s}$ (19 MMSCFD), recovering to $1.3 \mathrm{ST} \mathrm{m} / \mathrm{s}(4.0 \mathrm{MMSCFD})$ as production continues. For $k_{z} / k_{r}=0.05$, production peaks at 760 days at a rate of $7.0 \mathrm{ST} \mathrm{m} / \mathrm{s}(21.3 \mathrm{MMSCFD})$, recovering to $1.1 \mathrm{ST} \mathrm{m} 3 / \mathrm{s}(3.4 \mathrm{MMSCFD})$. Until $t=$ 800 days (low anisotropy) and $t=1050$ days (high anisotropy), cumulative averages are greater than for the reference case (no anisotropy). However, for both anisotropic cases, production rates level off between $2.4-2.8 \mathrm{ST} \mathrm{m} / \mathrm{s}$ (7.3 to 8.5 MMSCFD) during the recovery period, and cumulative averages remain below reference case values. Cumulative production, shown in Figure 31, reflects these trends, while water-to-gas ratios are similar for all three cases.

Spatial distributions of $S_{H}, S_{G}, T$, and $X_{i}$. The effects of anisotropy become clear when the distributions of system properties are examined. Figures $32 \mathrm{a}$ and $32 \mathrm{~b}$ assess the state of the system for the lower anisotropy (left column) and the high anisotropy (right column) cases at the time of first cavitation. For $k_{z} / k_{r}=0.10$ (Figure 32a), we see the presence of the typical secondary hydrate barrier, while in Figure $32 \mathrm{~b}$ the secondary hydrate has not yet appeared only days before the observed drop in production rate. However, a barrier forms in each case, and is seen in Figures $32 \mathrm{c}$ and $32 \mathrm{~d}$ several hundred days after the initial cavitation. In both cases, the secondary hydrate barrier has accumulated horizontal projections of secondary hydrate, reflecting channeling of flow along a preferred horizontal path. Gas saturations (Figure 33) show the accumulation of methane behind the obstruction for each case, while temperature profiles (Figure 34) show greater cooling with greater anisotropy, due to the lack of vertical transport of fluids from above and below the dissociating, and therefore cooling, hydrate mass. Inhibitor mass fractions (Figure 35), reveal regions of secondary hydrate formation (through increased mass fraction of salt adjacent to newly formed hydrate) and also show the stronger horizontal stratification of the system, with lower salt mass fractions horizontally aligned with regions of complete hydrate dissociation, reflecting transport of fresh water from the dissociated hydrate along those horizontal planes.

\section{Sensitivity variation (f): well spacing.}

The Class 3 reference case ${ }^{9}$, derived from the Class 2 case, also used a well spacing of 200 ha (500 acres). To assess sensitivity to well spacing, two new meshes were constructed, using the same discretization and underburden thickness as variations (d) and (e) applied to maximum radii of $562.2 \mathrm{~m}(a=100 \mathrm{ha})$ and $1128.4 \mathrm{~m}(a=400 \mathrm{ha})$.

Evolution of $\boldsymbol{Q}_{\boldsymbol{P}}, \boldsymbol{V}_{\boldsymbol{P}}, \boldsymbol{Q}_{\text {avg }}$, and $\boldsymbol{R}_{W G C}$. The evolution of production rate, $Q_{P}$, with varying well spacing, while initially similar to that seen in the Class 2 example, follows a dramatically different trend in the case of a Class 3 deposit. Figure 36 shows the evolution of production rate and average production over the full life of the reservoir. Although the more tightly spaced system initially achieves high rates at early times (4.6 ST m3/s or 14 MMSCFD at 200 days), after 1,000 days of production, the average production rate for the more widely-spaced system exceeds that of the 200 ha or 100 ha systems up to the point of reservoir depletion. The use of constant- $P$ production allows the system to "self-optimize" and achieve the degree of depressurization needed to maximize production rates, and to exploit a system with a significantly larger total quantity of hydrate per well with a single strategy. Cumulative production, seen in Figure 37, shows that all three well layouts produce a 
similar total quantity of gas over the first 1,200 days of production, while over the total life of the wells, the 400 ha case produces 4 times the quantity of gas (from an area 4 times larger) in approximately $2.5 x$ the time. Water-to-gas ratios, as in the Class 2 case, favor tighter well spacing.

Spatial distributions of $\boldsymbol{S}_{\boldsymbol{H}}, \boldsymbol{S}_{\boldsymbol{G}}, \boldsymbol{T}$, and $\boldsymbol{X}_{\boldsymbol{i}}$. Distributions of system properties are shown in Figures 38 through 41. The evolution of the tightly spaced system, shown in Figures 38a, c, and e, features the formation, movement, and dissolution of a single, relatively thin secondary hydrate barrier. Figures 39a, c, and e show the substantial quantity of gas entrained behind this barrier, while Figures $40 \mathrm{a} / \mathrm{c} / \mathrm{e}$ and $41 \mathrm{a} / \mathrm{c} / \mathrm{e}$ show the dramatic cooling and significant freshening associated with aggressive dissociation in a smaller-radius system. The 400 ha case, seen in panels $\mathrm{b}$, d, and $\mathrm{f}$ of Figures 38 through 41 , evolves with larger secondary hydrate barriers (Figure 38), and generates multiple traveling barriers that accumulate and combine together in the region between $r=10 \mathrm{~m}$ and $r=15 \mathrm{~m}$. Significant secondary hydrate formation is also seen within the remaining hydrate, in the region where the accumulated zone of free gas (Figure 39) intersects the hydrate zone. Temperatures (Figure 40) are warmer beyond the critical radius of $r=10-15 \mathrm{~m}$, and although similar degrees of freshening are seen, the thicker traveling barrier of the 400 ha case are visible in Figure 41 as localized regions of increased salinity.

\section{Effect of Permeable Boundaries on Production from Class 2 Deposits}

Simulation specifics. Previous simulations of production from unconfined Class 2 formations ${ }^{22}$ demonstrated the importance of impermeable upper and lower confining layers to the largely depressurization-driven process of gas production from hydrates. In reality, it is unlikely that one will find completely impermeable layers bounding a formation, and it is expected that production strategies may need to be adjusted to compensate. The problem of permeable boundaries is twofold. First, the accumulated gas often seen just below the overburden may migrate into overlying layers, escaping beyond the reach of production and in the worst case, entering the environment. Second, permeable boundaries reduce the effectiveness of depressurization and may add significant quantities of water to the reservoir, inhibiting hydrate dissociation and increasing water production rates.

To examine the worst-case scenario, we constructed a new system, otherwise identical to the Tigershark formation described in the previous sections but with $100 \mathrm{~m}$ of water-saturated overburden and underburden bounding the HBL. These layers were given intrinsic permeabilities of either $k=5 \mathrm{mD}$ and $k=50 \mathrm{mD}$ and a reduced porosity of $\phi=0.05$, approximating layers of semi-permeable shale. The top and bottom boundaries of the system were infinite boundaries at constant $T$ and $P$. This represents a worst-case scenario for a given permeability in that the entire upper and lower interfaces of the system have significant and uniform permeability.

We follow the same production strategy as in the Class 2 reference case, including the same mass production rates, production intervals, and data collection.

Evolution of $\boldsymbol{Q}_{\boldsymbol{P}}, \boldsymbol{Q}_{\boldsymbol{R}}, \boldsymbol{V}_{\boldsymbol{P}}$, and $\boldsymbol{R}_{W G C}$. The evolution of methane release from hydrate dissociation, $Q_{R}$, and production rate at the well, $Q_{P}$, are compared in Figure 42 . For $k=5 \mathrm{mD}$ (blue line), $Q_{P}$ follows a trend similar to the reference case (gray) for nearly 500 days, but then diverges as production fails to increase, soon decreasing slowly and continuously for over 5 years of attempted production (not shown). For $k=50 \mathrm{mD}$ (red line), the production rate drops rapidly, and at 175 days production slows dramatically as substantial secondary hydrate has formed along the inner radius of the hydrate zone. To jump start production, a conventional injection cycle was performed, and production was resumed with standard warm ocean water circulation at the top of the wellbore. The production rate does not recover, and additional secondary hydrate formation leads to the abandonment of production at 650 days.

The problem is clear in the evolution of $Q_{R}$ for both cases (green and orange lines). Gas release underperforms production at all times, and the gas generated through electrical heating or warm water circulation near the wellbore moves outward into the deposit to form secondary hydrate, as seen in previous studies examining pure thermal stimulation as a production strategy. ${ }^{9}$ The key feature in all of these cases is lack of strong depressurization throughout the formation. The 400 ha total (top and bottom) area of permeable boundary media allows substantial aqueous flow from outside the deposit in response to falling pressure within the formation. In Figure 43, water-to-gas ratios continuously to increase over the course of production, instead of decreasing over time, outpacing reference values immediately $(k=50 \mathrm{mD})$ or after 600 days $(k=5 \mathrm{mD})$. Cumulative gas production tracks the evolution of production rates, and is greatly reduced for both cases.

These results show that a modification of production strategy may be required to compensate for open formation boundaries. Depressurization-induced gas production, the most efficient method of production from hydrates, requires the pressure drops near the well are communicated effectively well into the reservoir. A fixed mass production rate, if used, would have be increased to generate significant pressure drops in the deposit, at least near the wellbore, and increased water production and/or secondary hydrate formation may then be an additional concern. In addition, colder water entering from the overburden (due to the geothermal gradient) comes in contact with gas produced from thermal stimulation in an environment without a strong decreasing trend in pressure, therefore promoting the formation of additional hydrate rather than assisting in gas release. The Class 2 sensitivity studies described earlier in this paper suggest that reducing well spacing may be the first solution to attempt, but a more detailed assessment of possible production strategies needs to be performed.

\section{Summary and Conclusions}

Production of methane gas from both Class 2 and Class 3 oceanic gas hydrates is shown to have great promise, if appropriate production strategies are implemented, for a range of deposits exhibiting a variety of physical conditions. A 
parametric sensitivity study reveals the following key insights about these variations and their consequences:

1) The production strategies outlined in Moridis and Reagan ${ }^{9,10}$ can apply to a wide range of Class 2 and Class 3 hydrate formations, with straightforward adjustments in the production strategy required to tailor production to the specific properties of the deposit.

2) For Class 2 formations, there is a strong dependence of production rate on porosity, with more porous formations requiring longer times to produce and equivalent quantity of gas.

3) For Class 2 formations, there is a mild dependence of production rate on the degree of anisotropy in formation permeability. Strongly anisotropic systems may see a delay in cumulative gas production, but water-to-gas ratios are still favorable, and production delays may be compensated for through judicious adjustment of production rates and strategy.

4) For Class 2 formations, there is a strong dependence on well spacing, with tighter configurations of wells producing gas at higher rates over shorter time spans.

5) For Class 3 formations, there is a significant dependence of both production rate and the efficiency of production on underlying porosity.

6) For Class 3 formations, there is a significant effect of anisotropy in the evolution of the system during production that may not be mitigated solely through the "self-regulating" constant-pressure production approach.

7) For Class 3 formations, there is a significant effect of well spacing on production rate, with some trends contrary to those seen in the Class 2 case. Increasing the area per well by a factor of 4 produces four times the quantity of gas in $2.5 x$ the time, and therefore careful selection of well spacing is required for efficient and economical large-scale production.

8) Open boundaries present a new challenge to production from hydrates, as infiltration of water from outside the deposit can impare the ability of constant-rate Class-2 production methods to induce strong depressurization within the deposit and greatly reduce production rates.

\section{Nomenclature}

$\Delta r=$ Radial increment $(\mathrm{m})$

$\Delta z=$ Vertical discretization, i.e., in the $z$-direction (m)

$C=\operatorname{specific}$ heat $(\mathrm{J} / \mathrm{kg} / \mathrm{K})$

$F_{A}=$ area factor (dimensionless)

$h=$ thickness of the hydrate bearing layer $(\mathrm{m})$

$H_{W}=$ specific enthalpy of water circulating in the well $(\mathrm{J} / \mathrm{kg})$

$k=$ intrinsic permeability $\left(\mathrm{m}^{2}\right)$

$k_{\text {eff }}=$ effective permeability, accounting for the presence of ice and/or hydrate $\left(\mathrm{m}^{2}\right)$

$k_{r}=$ relative permeability $\left(\mathrm{m}^{2}\right)$

$k_{\Theta}=$ thermal conductivity $(\mathrm{W} / \mathrm{m} / \mathrm{K})$

$k_{\Theta R D}=$ thermal conductivity of dry porous medium $(\mathrm{W} / \mathrm{m} / \mathrm{K})$

$k_{\Theta R W}=$ thermal conductivity of fully saturated porous medium $(\mathrm{W} / \mathrm{m} / \mathrm{K})$

$M_{W}=$ cumulative mass of water removed $(\mathrm{kg})$

$N_{H}=$ hydration number

$P=$ pressure $(\mathrm{Pa})$

$P_{0}=$ initial pressure in hydrate-bearing sediments $(\mathrm{Pa})$

$P_{Q}=$ pressure at the quadruple point $(\mathrm{Pa})$

$Q_{\Theta}=$ rate of heat injection into the formation next to the well ( $\mathrm{W} / \mathrm{m}$ of wellbore)

$Q_{I}=$ mass rate of injected warm water at the well $(\mathrm{kg} / \mathrm{s})$

$Q_{M}=$ mass rate of fluid withdrawal at the well $(\mathrm{kg} / \mathrm{s})$

$Q_{P}=$ volumetric rate of $\mathrm{CH}_{4}$ production at the well $\left(\mathrm{ST} \mathrm{m}^{3} / \mathrm{s}\right)$

$Q_{R}=$ volumetric rate of $\mathrm{CH}_{4}$ release from hydrate dissociation into the reservoir $\left(\mathrm{ST} \mathrm{m}^{3} / \mathrm{s}\right)$

$Q_{V}=$ rate of $\mathrm{CH}_{4}$ release from hydrate dissociation $\left(\mathrm{ST} \mathrm{m}^{3} / \mathrm{s}\right)$

$r, z=$ coordinates $(\mathrm{m})$

$r_{c}=$ critical radius of maximum activity around the wellbore $(\mathrm{m})$

$r_{D}=$ radius of the dissociation around the well $(\mathrm{m})$

$r_{w}=$ radius of the well assembly $(\mathrm{m})$

$r_{\text {max }}=$ maximum radius of the simulation domain $(\mathrm{m})$

$R_{W G C}=$ ratio of water removed per volume of gas produced

$S=$ phase saturation

$t=$ time $(\mathrm{s})$

$T=$ temperature $\left(\mathrm{K}\right.$ or $\left.{ }^{\circ} \mathrm{C}\right)$

$V_{R}=$ cumulative volume of $\mathrm{CH}_{4}$ released from hydrate dissociation $\left(\mathrm{ST} \mathrm{m}^{3}\right)$

$V_{P}=$ cumulative volume of $\mathrm{CH}_{4}$ released into the ocean through the annular gravel pack $\left(\mathrm{ST} \mathrm{m}^{3}\right)$

$X_{i}=$ water salinity (mass fraction) 


\section{Greek Symbols}

$$
\begin{aligned}
& \lambda=\text { van Genuchten exponent } \\
& \phi=\text { porosity }
\end{aligned}
$$

\section{Subscripts and Superscripts}

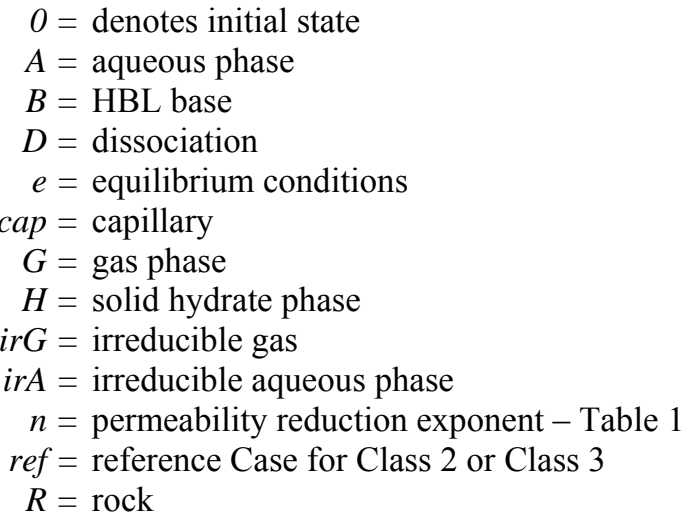

\section{Acknowledgment}

This work was supported by the Assistant Secretary for Fossil Energy, Office of Natural Gas and Petroleum Technology, through the National Energy Technology Laboratory, under the U.S. Department of Energy, Contract No. DE-AC0205CH11231. Thanks are extended to Ray Boswell and Kelly Rose of DOE-NETL for making the Tigershark data available to the authors. The critical contributions of Tim Collett of USGS in the analysis and evaluation of the geological and geophysical data are gratefully acknowledged.

\section{References}

1. Sloan, E.D., Clathrate Hydrates of Nautral Gases. Marcel Decker, Inc., New York, NY (1998).

2. Milkov, A. V., "Global estimates of hydrate-bound gas in marine sediments: How much is really out there," Earth Science Reviews 66(3), 183 (2004).

3. Klauda, J.B., Sandler, S.I., "Global distribution of methane hydrate in ocean sediment," Energy \& Fuels 19, 469 (2005).

4. Makogon, Y.F., "Gas hydrates: frozen energy," Recherche 18(192), 1192 (1987).

5. Dallimore, S.R., Collett, T.S., Eds., "Scientific Results from the Mallik 2002 Gas Hydrate Production Research Well Program, Mackenzie Delta, Northwest Territories, Canada," Geological Survey of Canada Bulletin 585 (2005).

6. Makagon, Y.F., Hydrates of Hydrocarbons. Penn Well Publishing Co. Tulsa, OK. (1997).

7. Moridis, G.J., and Collett, T.S.: "Strategies for gas production from hydrate accumulations under various geologic conditions", Report LBNL-52568, Lawrence Berkeley National Laboratory, Berkeley, CA (2003).

8. Moridis, G.J., Kowalsky, M.B., Pruess, K., "Depressurization-induced gas production from Class 1 hydrate deposits," (LBNL-59780) SPE 97266, 2005 SPE Annual Technical Conference and Exhibition, Dallas, Texas, U.S.A., October 9-12, 2005.

9. Moridis, G.J and M.T. Reagan, "Strategies for Gas Production From Oceanic Class 3 Hydrate Accumulations" OTC 18865, 2007 Offshore Technology Conference, Houston, Texas, U.S.A., 30 April-3 May 2007.

10. Moridis, G.J and M.T. Reagan, "Gas Production From Oceanic Class 2 Hydrate Accumulations" OTC 18866, 2007 Offshore Technology Conference, Houston, Texas, U.S.A., 30 April-3 May 2007.

11. Moridis, G.J., Sloan, E.D., "Gas Production Potential of Disperse Low-Saturation Hydrate Accumulations in Oceanic Sediments," (LBNL-52586) in press, J. Energy Conversion \& Management (2007).

12. Moridis, G.J., Kowalsky, M.B., "Gas Production from Unconfined Class 2 Hydrate Accumulations in the Oceanic Subsurface," (LBNL-57299) in Economic Geology of Natural Gas Hydrates, M. Max, A.H. Johnson, W.P. Dillon and T. Collett, Eds., Kluwer Academic/Plenum Publishers (2006).

13. Smith, S., Boswell, R., Collett, T., Lee, M., Jones, E., "Alaminos Canyon Block 818: A Documented Example of Gas Hydrate Saturated Sand in the Gulf of Mexico," Fire In The Ice, NETL Methane Hydrates R\&D Program Newsletter, Fall 2006.

14. Collett, T.S., Lee, M.W., Well Log Analysis: Tiger Shark AC 818 No. 1, U.S. Geological Survey, internal memo (2006).

15. Moridis, G.J., Kowalsky, M.B., "Response of Oceanic Hydrate-Bearing Sediments to Thermal Stresses," (LBNL-60150) OTC 18193, 2006 Offshore Technology Conference, Houston, Texas, U.S.A., May 1-4, 2006.

16. Kim, H.C., Bishnoi, P.R., Heidemann, R.A., and Rizvi, S.S.H.: "Kinetics of Methane Hydrate Decomposition", Chem. Eng. Sci., 42(7), 1645 (1987).

17. Moridis, G.J., Kowalsky, M.B., Pruess, K., "TOUGH-Fx/HYDRATE v1.0 User's Manual: A Code for the Simulation of System Behavior in Hydrate-Bearing Geologic Media," Report LBNL-3185, Lawrence Berkeley National Laboratory, Berkeley, CA (2005)

18. Clarke, M.A., and Bishnoi, P.R.: "Determination of the Intrinsic Rate of Methane Gas Hydrate Decomposition", Chem. Eng. Sci., 55, 4869 (2000).

19. Moridis, G.J., T.S. Collett, S.R. Dallimore, T. Inoue and T. Mroz, “Analysis and Interpretation of the Thermal Test of Gas Hydrate Dissociation in the JAPEX/JNOC/GSC et al. Mallik 5L-38 Gas Hydrate Production Research Well”, in Scientific Results from the 
Mallik 2002 Gas Hydrate Production Research Well Program, Mackenzie Delta, Northwest Territories, Canada. Geological Survey of Canada, Bulletin 585, S.R. Dallimore and T. Collett, Editors, 2005 (LBNL-57296).

20. Kowalsky, M.B., Moridis, G.J., "Comparison of Kinetic and Equilibrium Reaction Models in Simulating the Behavior of Gas Hydrates in Porous Media, “(LBNL-60361) in press, J. Energy Conversion \& Management (2006).

21. Wright, J.F., Dallimore, S.R., and Nixon, F.M.: "Influences of Grain Size and Salinity on Pressure-Temperature Thresholds for Methane Hydrate Stability in JAPEX/JNOC/GSC Mallik 2L-38 Gas Hydrate Research-Well Sediments", in Scientific Results from JAPEX/JNOC/GSC Mallik 2L-38 Gas Hydrate Research-Well, Mackenzie Delta, Northwest Territories, Canada. Dallimore, S.R., Uchida, T., and Collett, T.S., Eds., Geological Survey of Canada Bulletin 544, 229 (1999).

22. Moridis, G.J., Kowalsky, M.B., "Gas Production from Unconfined Class 2 Hydrate Accumulations in the Oceanic Subsurface," (LBNL-57299) in Economic Geology of Natural Gas Hydrates, M. Max, A.H. Johnson, W.P. Dillon and T. Collett, Eds., Kluwer Academic/Plenum Publishers (2006).

23. Moridis, G.J., Seol, Y., Kneafsey, T., "Studies of Reaction Kinetics of Methane Hydrate Dissociation in Porous Media", Report LBNL-57298, Lawrence Berkeley National Laboratory, Berkeley, CA (2005).

24. van Genuchten, M.Th.: "A Closed-Form Equation for Predicting the Hydraulic Conductivity of Unsaturated Soils", Soil Sci. Soc., 44, $892(1980)$. 


\begin{tabular}{|c|c|}
\hline \multicolumn{2}{|c|}{ Table 1 - Hydrate Deposit Properties } \\
\hline Parameter & Value \\
\hline Class 2 Water zone thickness & $15 \mathrm{~m}$ \\
\hline Hydrate zone thickness & $18.25 \mathrm{~m}$ \\
\hline Initial pressure $P_{B}$ & $3.3 \times 10^{7} \mathrm{~Pa}$ \\
\hline $\begin{array}{l}\text { Initial temperature } T_{B} \\
\text { (at base of } \mathrm{HBL} \text { ) }\end{array}$ & $294.15 \mathrm{~K}\left(21^{\circ} \mathrm{C}\right)$ \\
\hline Gas composition & $100 \% \mathrm{CH}_{4}$ \\
\hline Initial saturations in the $\mathrm{HBL}$ & $S_{H}=0.7, S_{A}=0.3$ \\
\hline Water salinity (mass fraction) & 0.03 \\
\hline Initial saturations in the HBL & $S_{H}=0.7, S_{A}=0.3$ \\
\hline $\begin{array}{l}\text { Initial Intrinsic permeability } \mathrm{k}_{\mathrm{r}}=\mathrm{k}_{\mathrm{z}} \\
\text { (HBL and water zone) }\end{array}$ & $\begin{array}{l}750 \mathrm{mD}\left(=7.5 \times 10^{-13}\right. \\
\left.\mathrm{m}^{2}\right)\end{array}$ \\
\hline $\begin{array}{l}\text { Intrinsic permeability } \mathrm{k}_{\mathrm{r}}=\mathrm{k}_{\mathrm{z}} \\
\text { (overburden \& underburden) }\end{array}$ & $0 \mathrm{mD}, 5 \mathrm{mD}, 50 \mathrm{mD}$ \\
\hline $\begin{array}{l}\text { Grain density } \rho_{R} \\
\text { (all formations) }\end{array}$ & $2750 \mathrm{~kg} / \mathrm{m}^{3}$ \\
\hline $\begin{array}{l}\text { Class } 2 \text { Initial mass production } \\
\text { rate } \mathrm{Q}_{\mathrm{M} 0}\end{array}$ & $\begin{array}{l}18.955 \mathrm{~kg} / \mathrm{s} \\
(=10,000 \mathrm{BPD})\end{array}$ \\
\hline Class 3 Initial bottomhole pressure & $3.0 \mathrm{MPa}$ \\
\hline $\begin{array}{l}\text { Composite thermal } \\
\text { conductivity model }^{23}\end{array}$ & $\begin{array}{l}k_{\Theta C}=k_{\Theta R D} \\
+\left(S_{A}^{1 / 2}+S_{H}^{1 / 2}\right)\left(k_{\Theta R W}-\right. \\
\left.k_{\Theta R D}\right)+\phi S_{1} k_{\Theta}\end{array}$ \\
\hline $\begin{array}{l}\text { Dry thermal conductivity } \\
k_{\Theta R D} \text { (all formations) }\end{array}$ & $0.5 \mathrm{~W} / \mathrm{m} / \mathrm{K}$ \\
\hline $\begin{array}{l}\text { Wet thermal conductivity } \\
k_{\Theta R W} \text { (all formations) }\end{array}$ & $3.1 \mathrm{~W} / \mathrm{m} / \mathrm{K}$ \\
\hline Capillary pressure model ${ }^{17,24}$ & $P_{\text {cap }}=-P_{0}\left[\left(S^{*}\right)^{-1 / \lambda}-1\right]^{\lambda}$ \\
\hline & $S^{*}=\frac{\left(S_{A}-S_{i r A}\right)}{\left(S_{m \times A}-S_{i r A}\right)}$ \\
\hline$S_{\text {irA }}$ & 1 \\
\hline$\lambda$ & 0.45 \\
\hline$P_{0}$ & $10^{5} \mathrm{~Pa}$ \\
\hline Relative permeability & $k_{r A}=\left(S_{A}\right)^{n}$ \\
\hline Model $^{14}$ & $k_{r G}=\left(S_{G}\right)^{n}$ \\
\hline & $S_{A}^{*}=\left(S_{A}-S_{i r A}\right) /\left(1-S_{i r A}\right)$ \\
\hline & $\begin{array}{l}S_{G}{ }^{n}=\left(S_{G}-S_{i r G}\right) /\left(1-S_{i r A}\right) \\
\text { OPM model }\end{array}$ \\
\hline$n$ (from Moridis et al. $^{19}$ ) & 3.572 \\
\hline$S_{i r G}$ & 0.02 \\
\hline$S_{i r A}$ & 0.25 \\
\hline
\end{tabular}




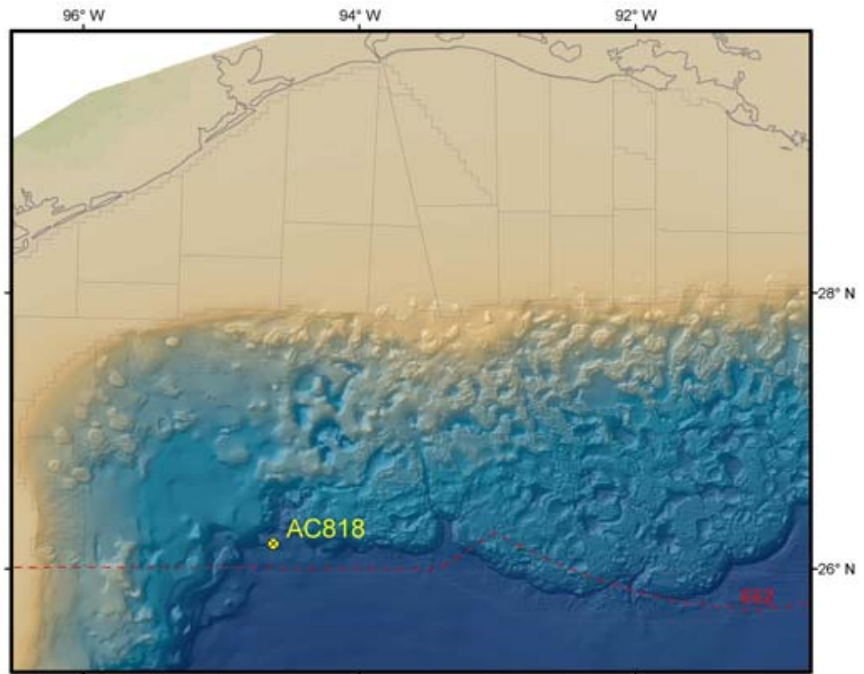

Figure 1 - Approximate location of the Tigershark exploratory well in the Alaminos Canyon block $818^{13}$.

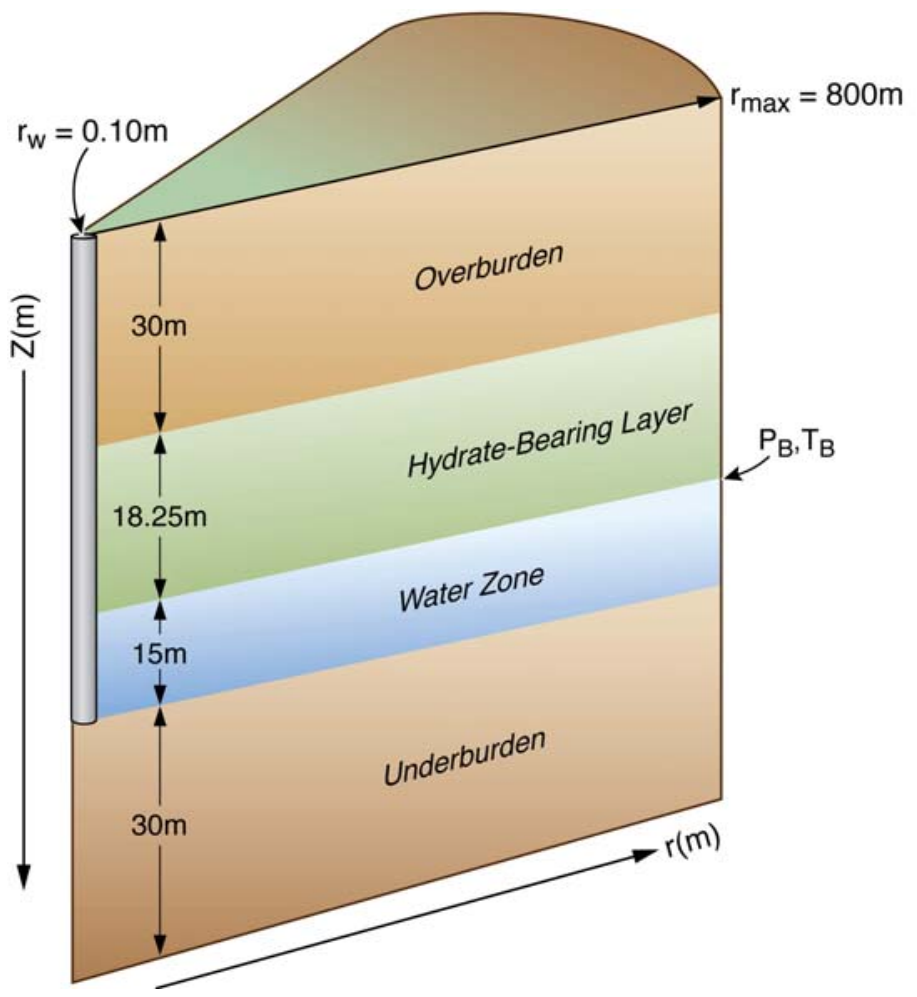

Figure 2 - A schematic of the Class 2 hydrate deposit simulated in this study. 


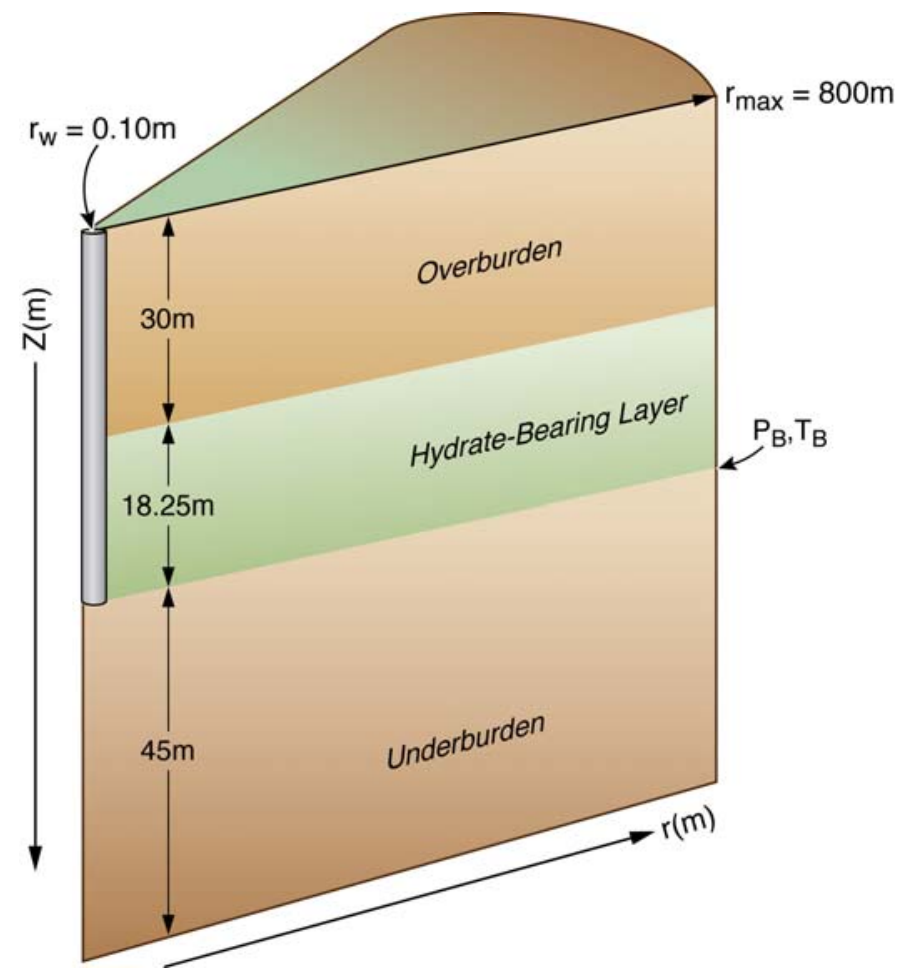

Figure 3 - A schematic of the Class 3 hydrate deposit simulated in this study.

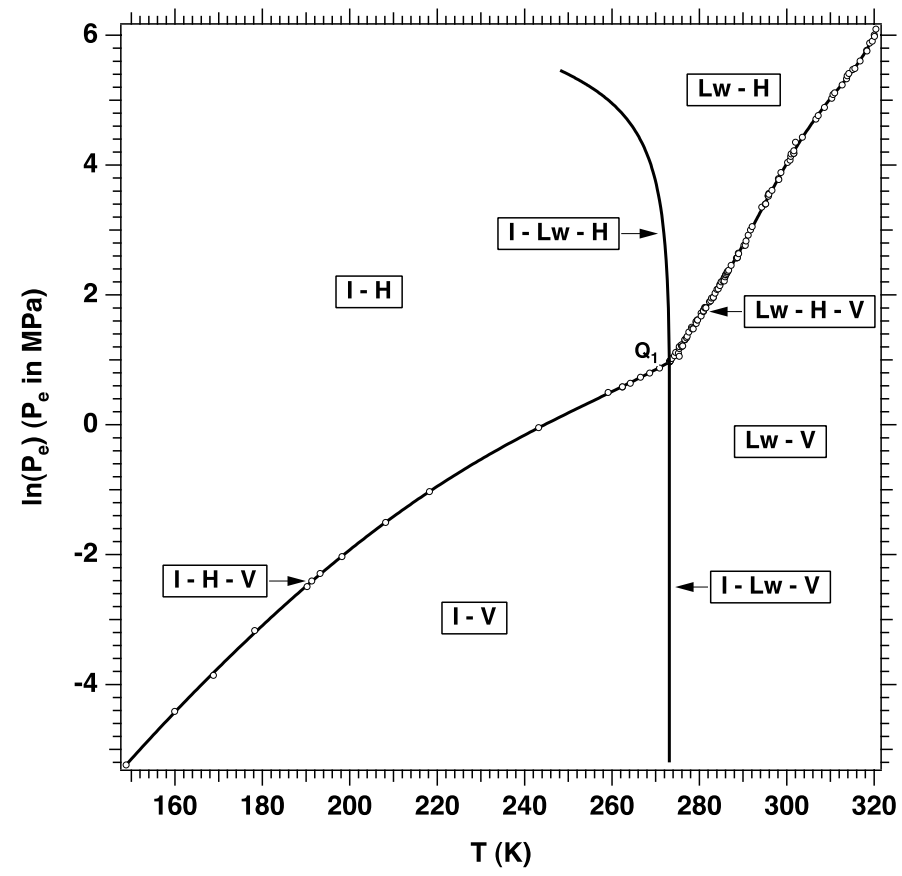

Figure 5 - Pressure-temperature equilibrium relationship in the phase diagram of the water- $\mathrm{CH}_{4}-\mathrm{hydrate}$ system ${ }^{17}$ (Lw: $\mathrm{Liquid}_{\text {water; }}$ H: Hydrate; V: Vapor (gas phase); I: Ice; $Q_{1}$ : Quadruple point = I + Lw + H + V) 


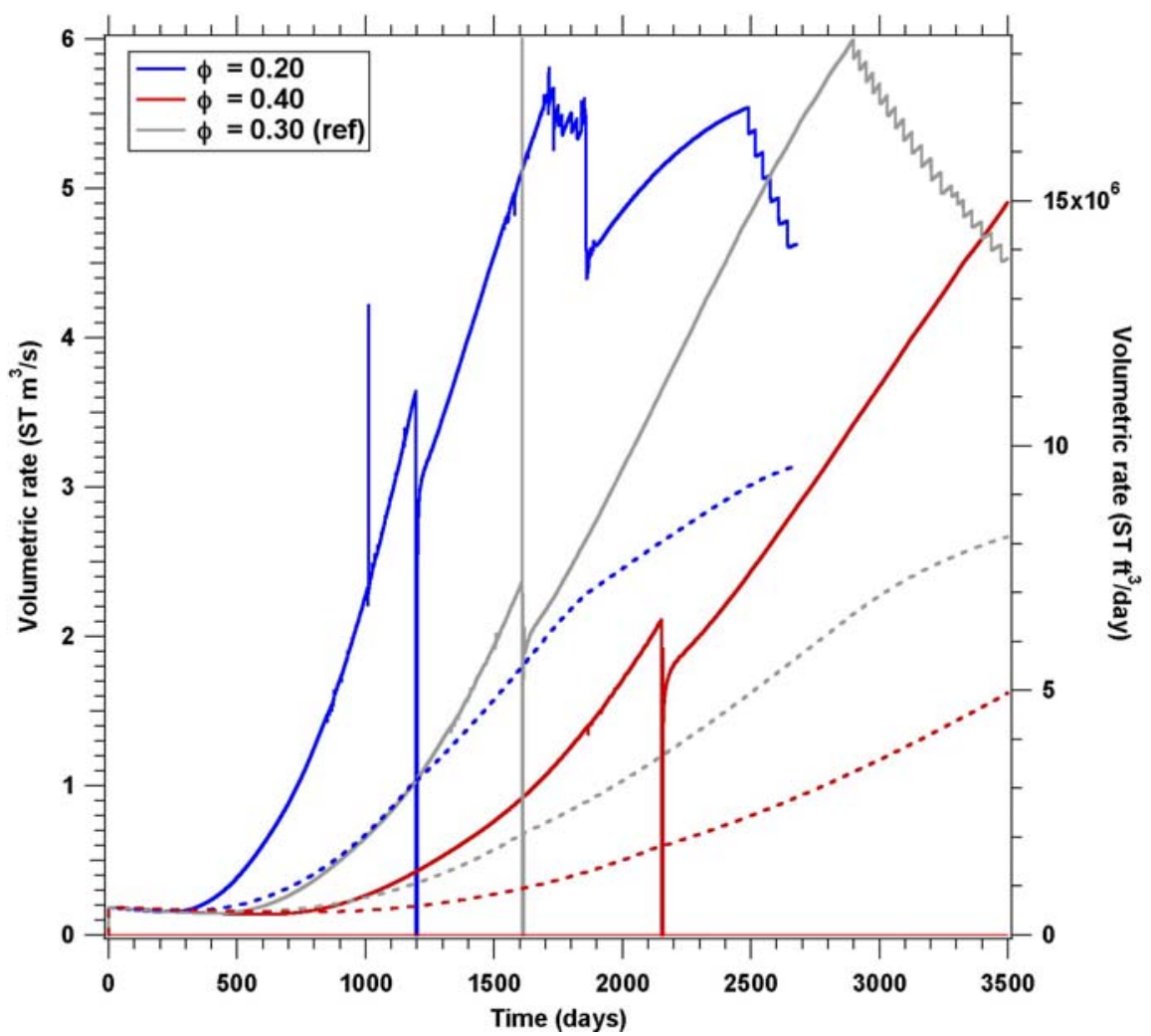

Figure 6 - Rate of $\mathrm{CH}_{4}$ production at the well, $Q_{P}$ (solid lines), and the evolution of average production (dotted lines) during production from a Class 2 oceanic deposit at varying porosity.

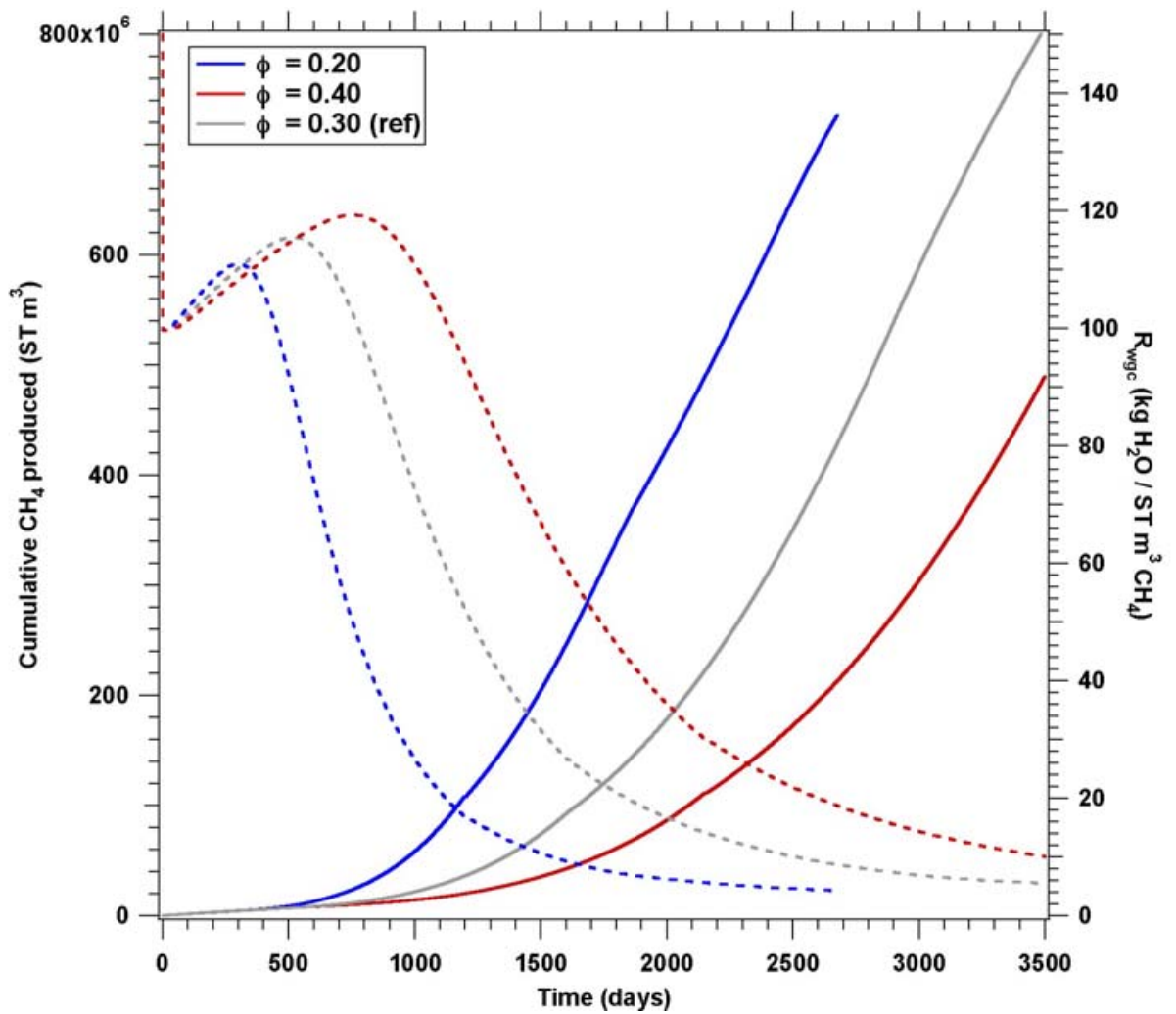

Figure 7 - Cumulative volume of $\mathrm{CH}_{4}$ produced at the well, $V_{P}$ (solid lines), and the cumulative water-to-gas ratio, $\boldsymbol{R}_{W G C}$ (dotted lines), during production from a Class 2 oceanic deposit at varying porosity. 


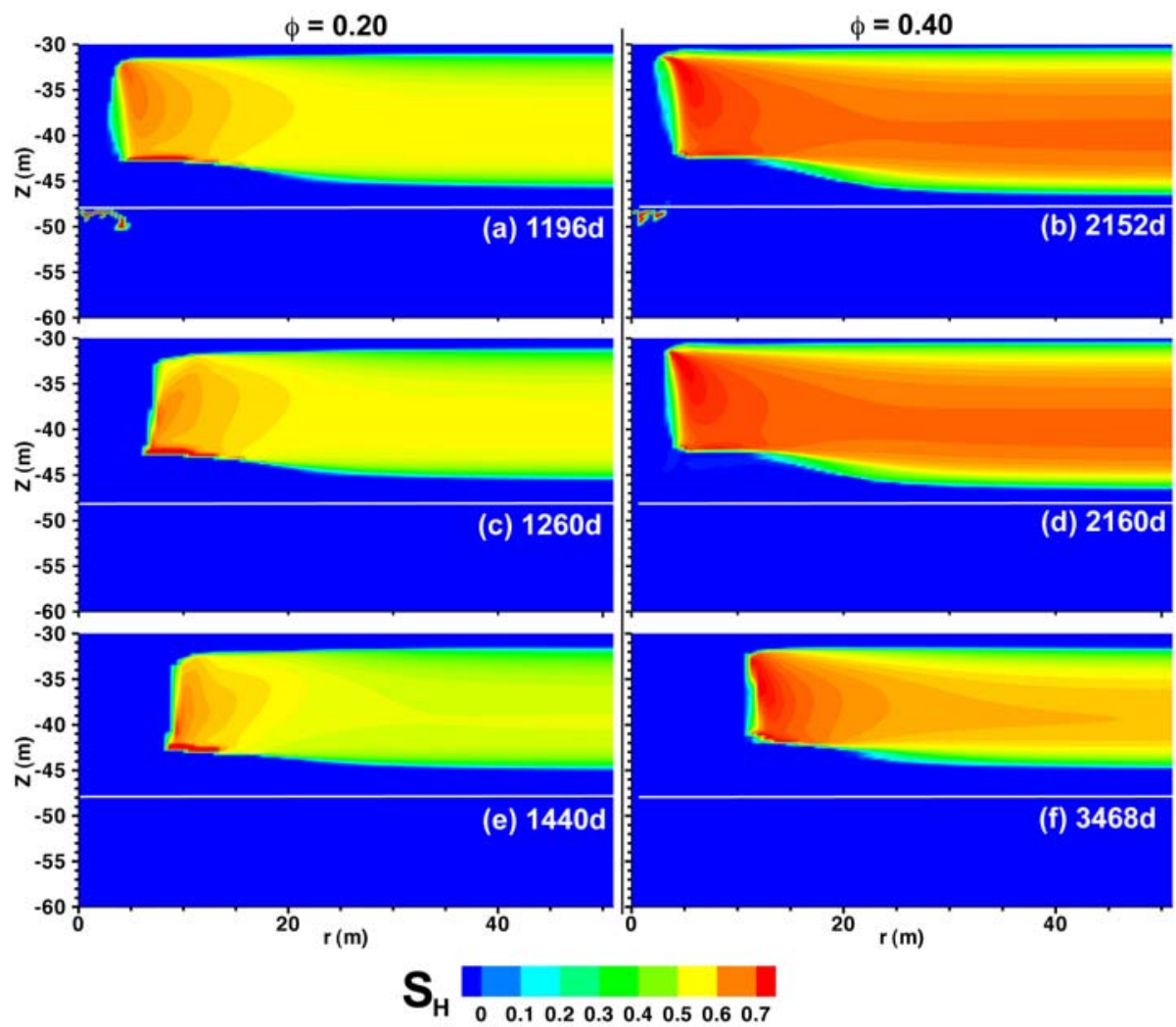

Figure 8 - Evolution of the spatial distribution of $S_{H}$ during gas production from the Class 2 oceanic hydrate deposit with varying porosity.

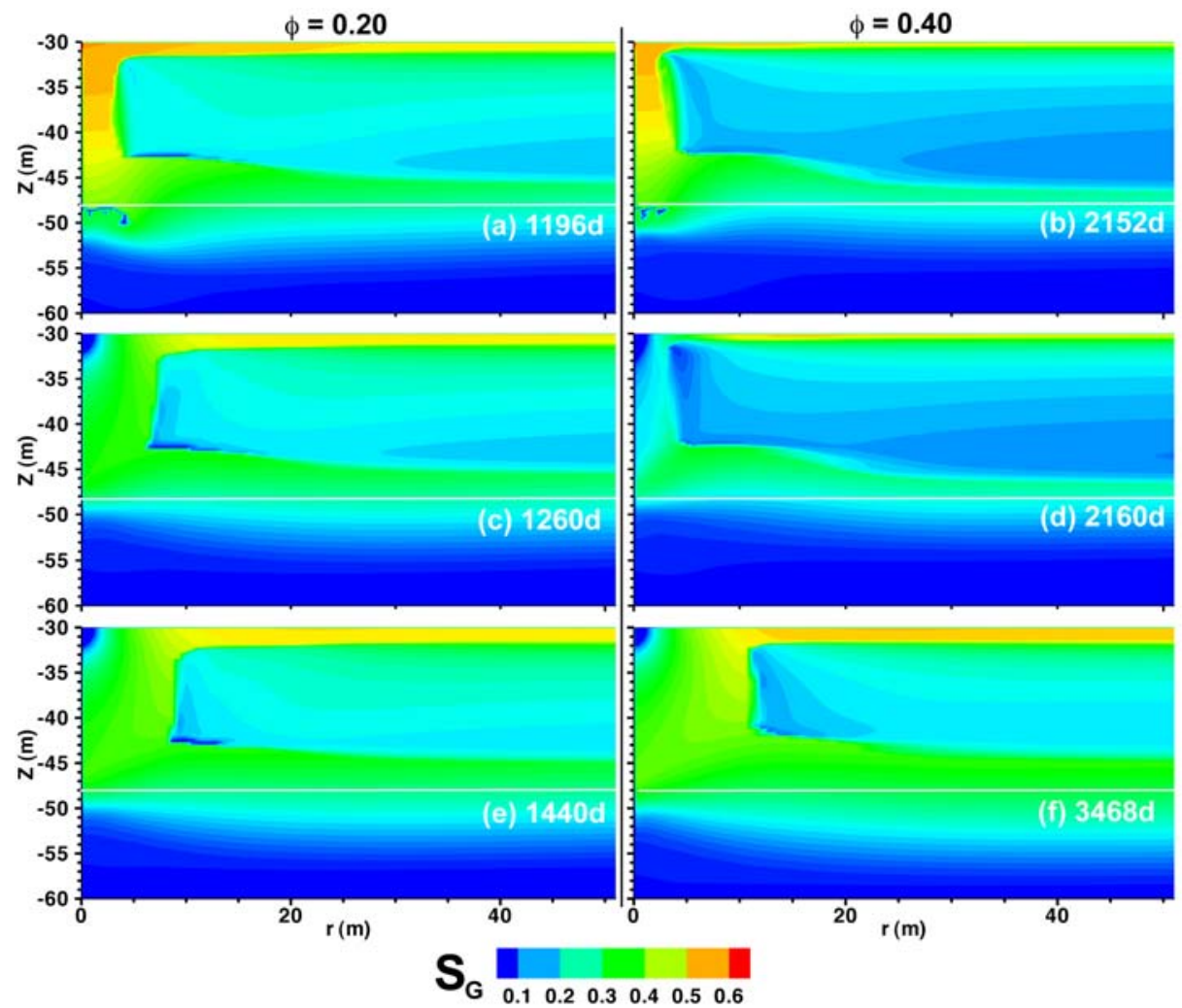

Figure 9 - Evolution of the spatial distribution of $S_{G}$ during gas production from the Class 2 oceanic hydrate deposit with varying porosity. 


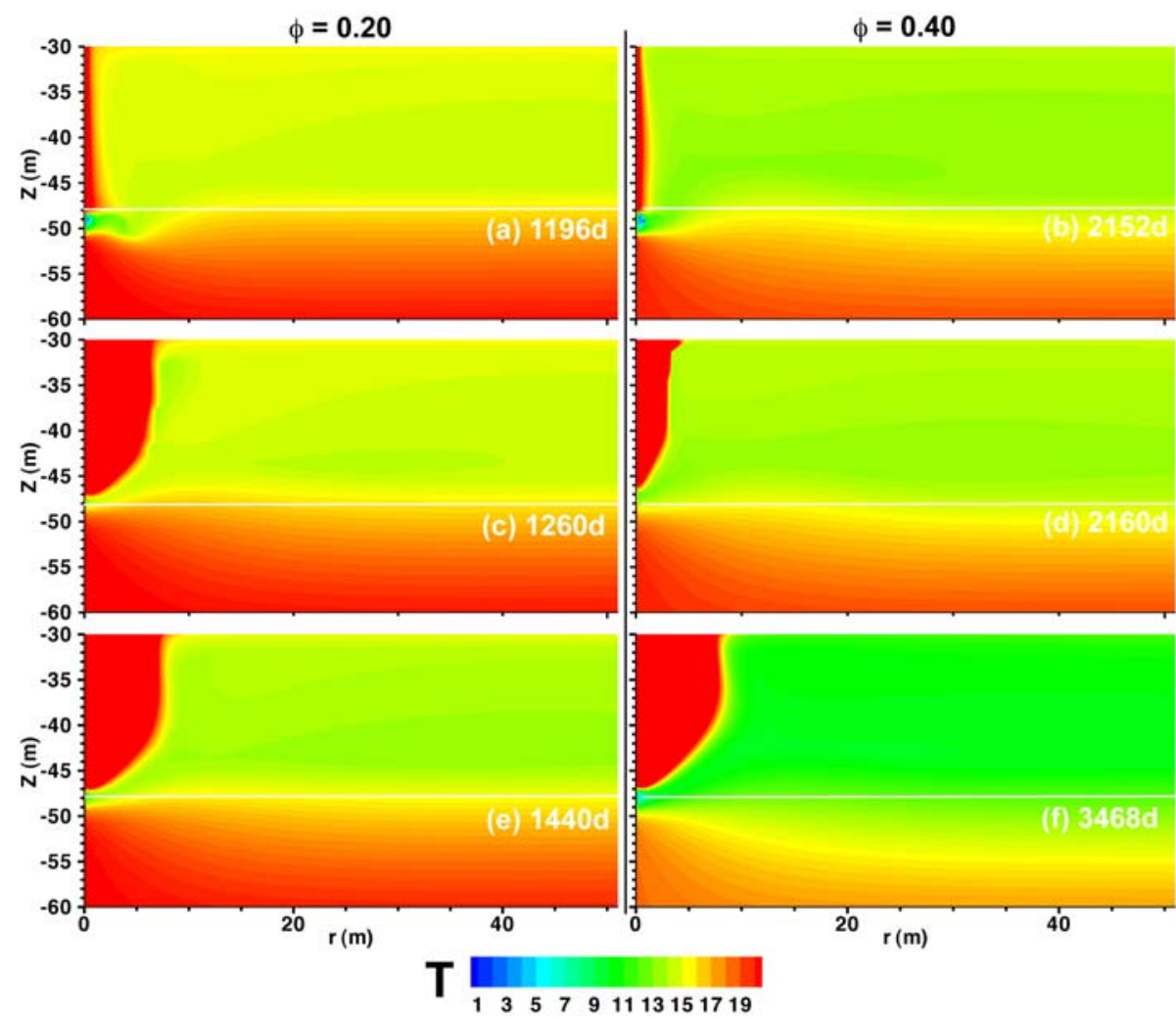

Figure 10 - Evolution of the spatial distribution of $T$ during gas production from the Class 2 oceanic hydrate deposit with varying porosity.

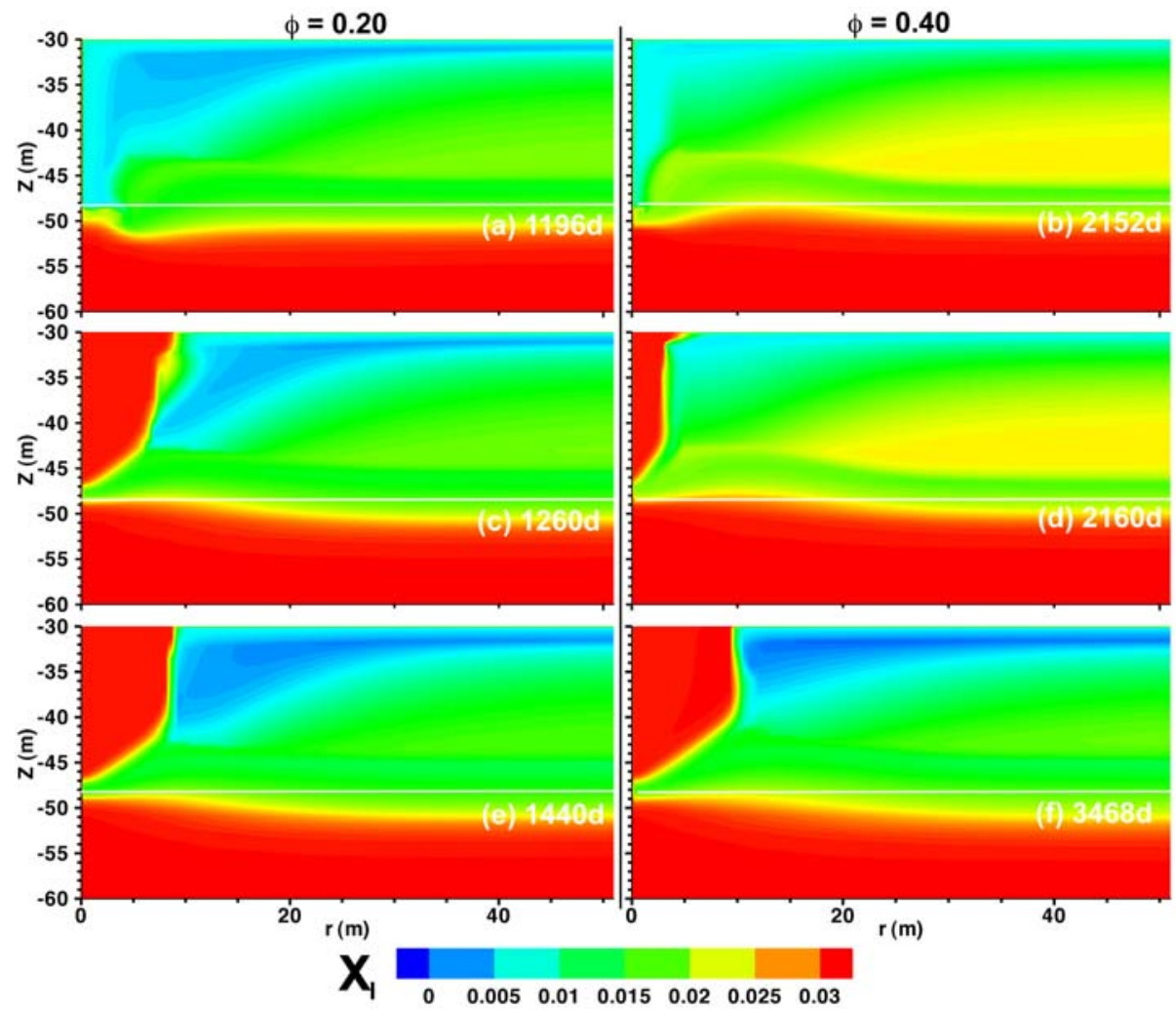

Figure 11 - Evolution of the spatial distribution of $X_{l}$ during gas production from the Class 2 oceanic hydrate deposit with varying porosity. 


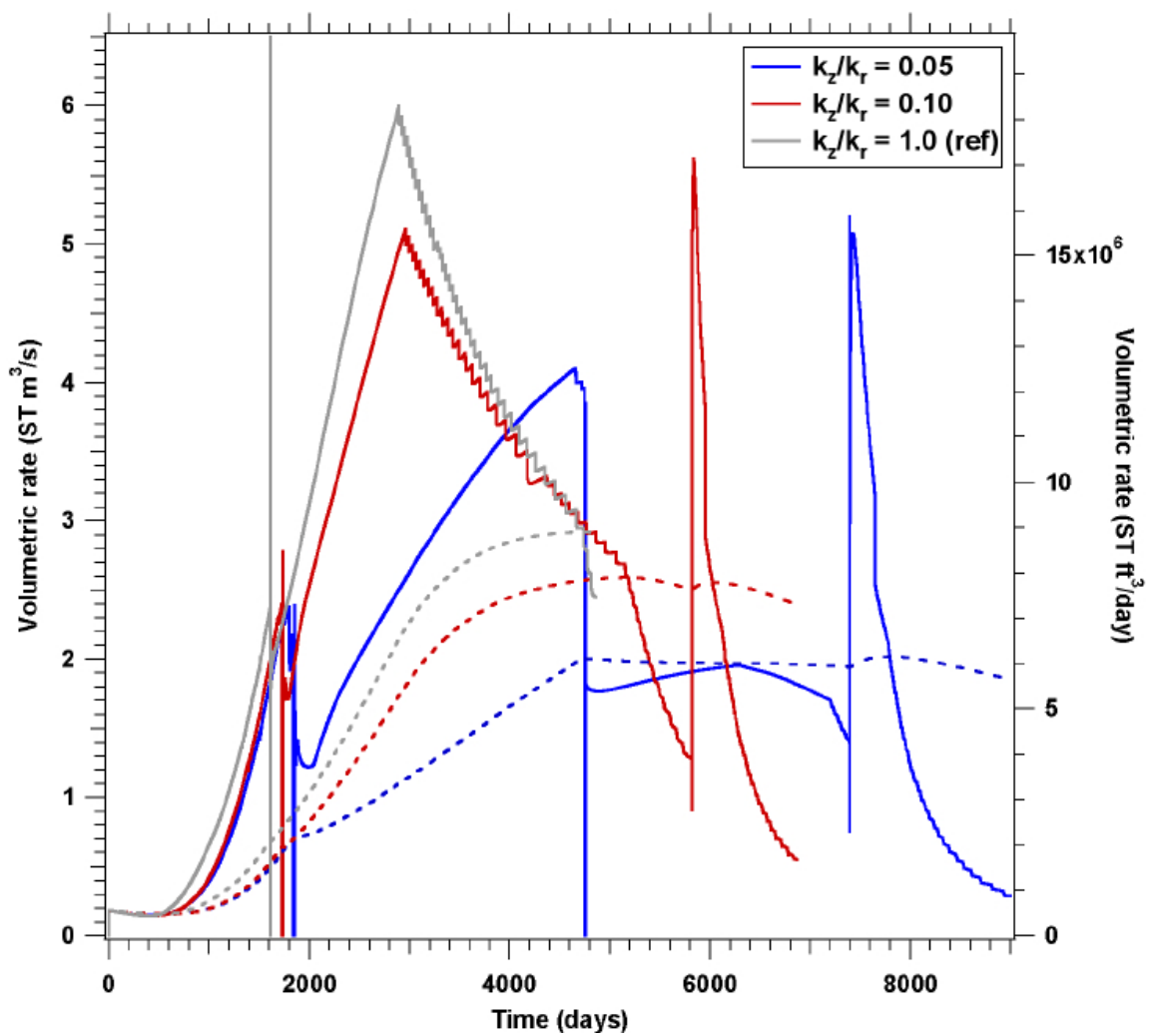

Figure 12 - Rate of $\mathrm{CH}_{4}$ production at the well, $Q_{P}$ (solid lines), and the evolution of average production (dotted lines) during production from a Class 2 oceanic deposit with varying anisotropy.

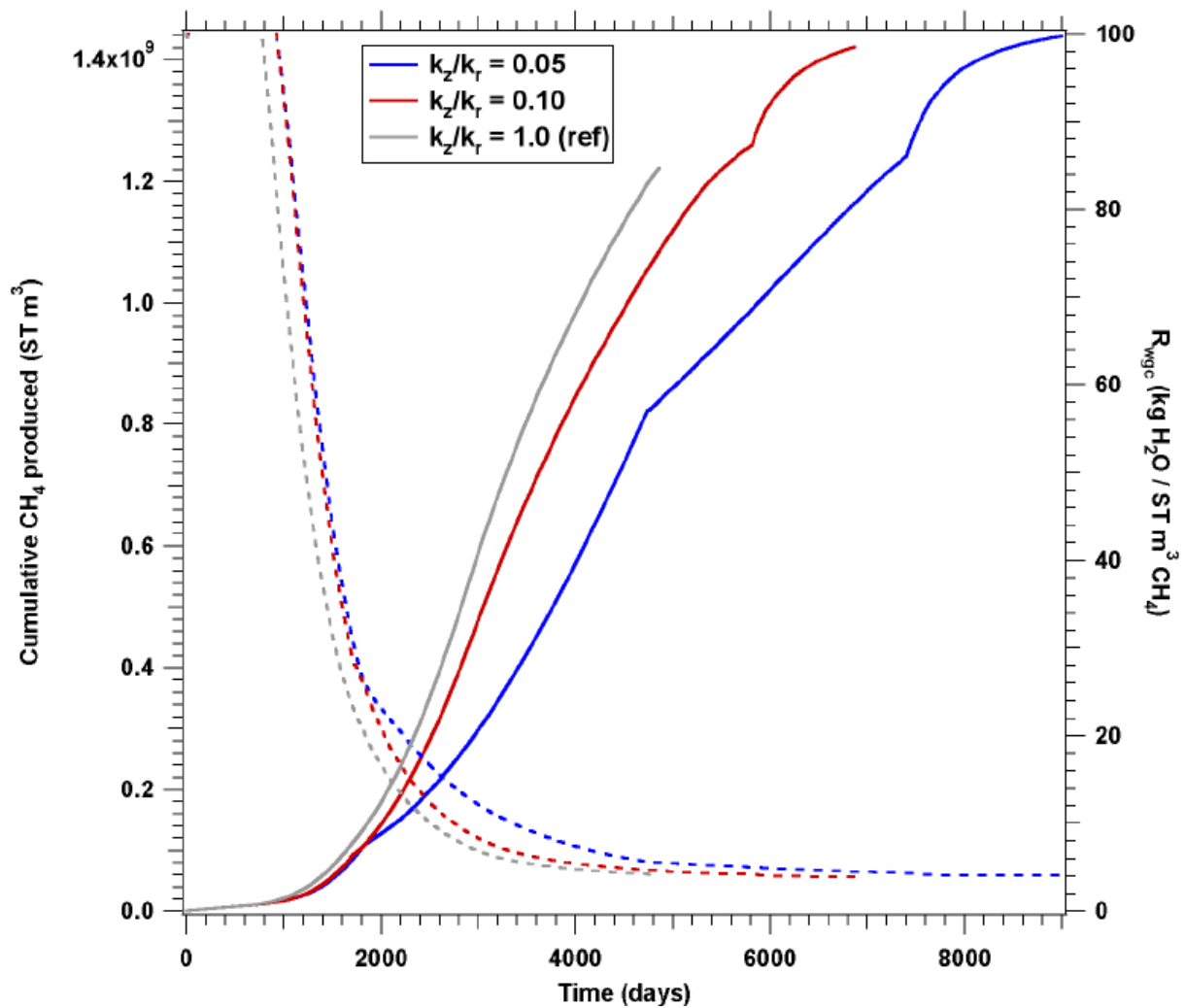

Figure 13 - Cumulative volume of $\mathrm{CH}_{4}$ produced at the well, $V_{P}$ (solid lines), and the cumulative water-to-gas ratio, $R_{\text {WGC }}$ (dotted lines), during production from a Class 2 oceanic deposit with varying anisotropy. 


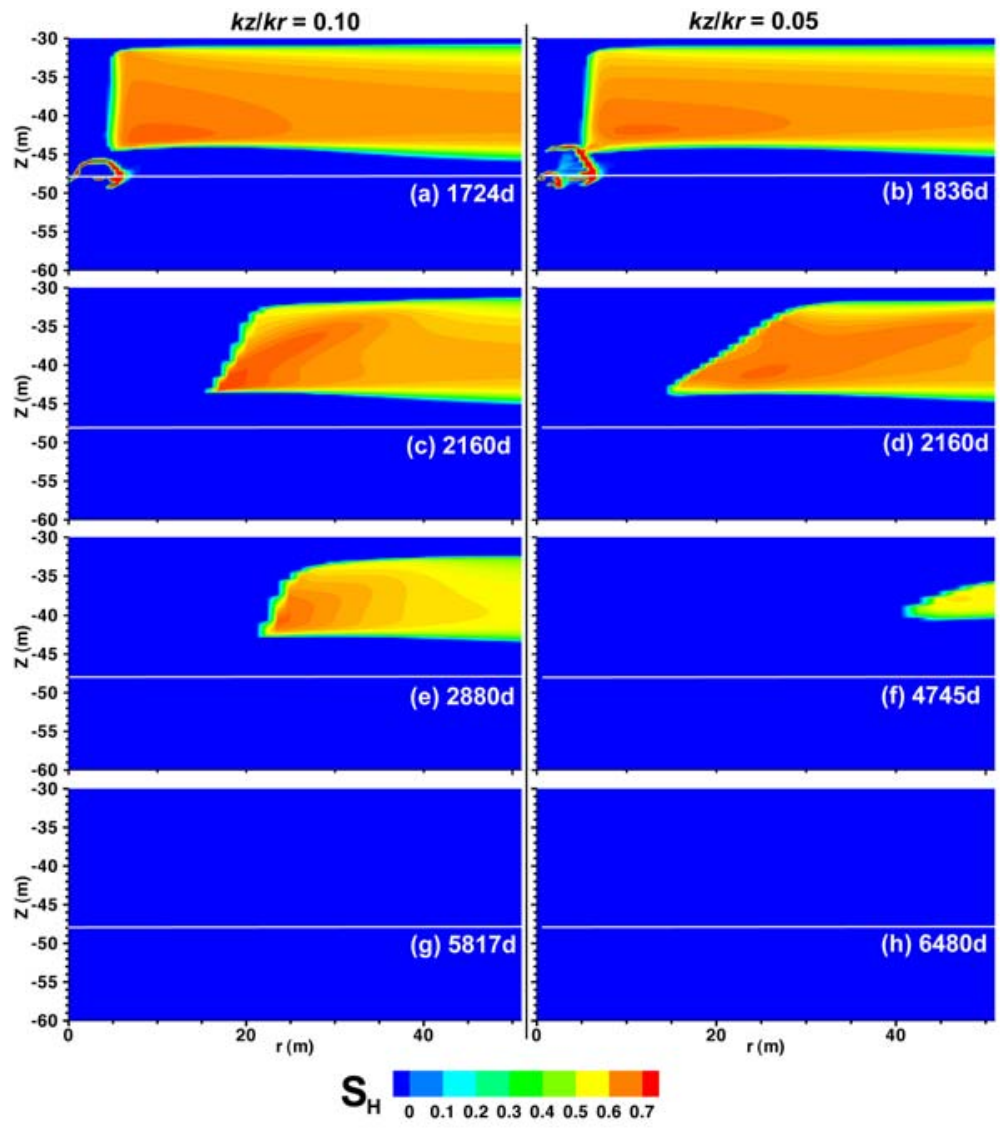

Figure 14 - Evolution of the spatial distribution of $S_{H}$ during gas production from the Class 2 oceanic hydrate deposit with varying anisotropy.

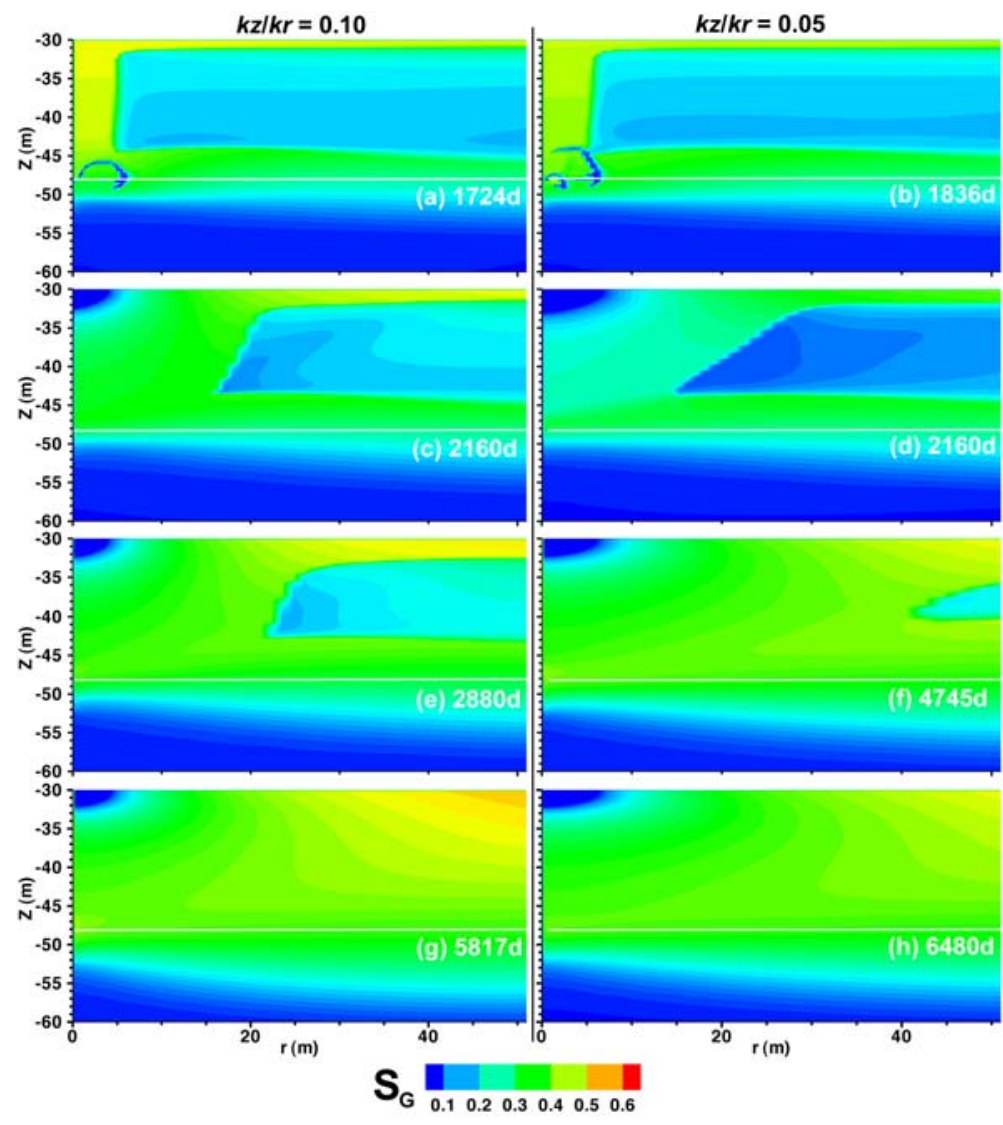

Figure 15 - Evolution of the spatial distribution of $S_{G}$ during gas production from the Class 2 oceanic hydrate deposit with varying 
anisotropy.

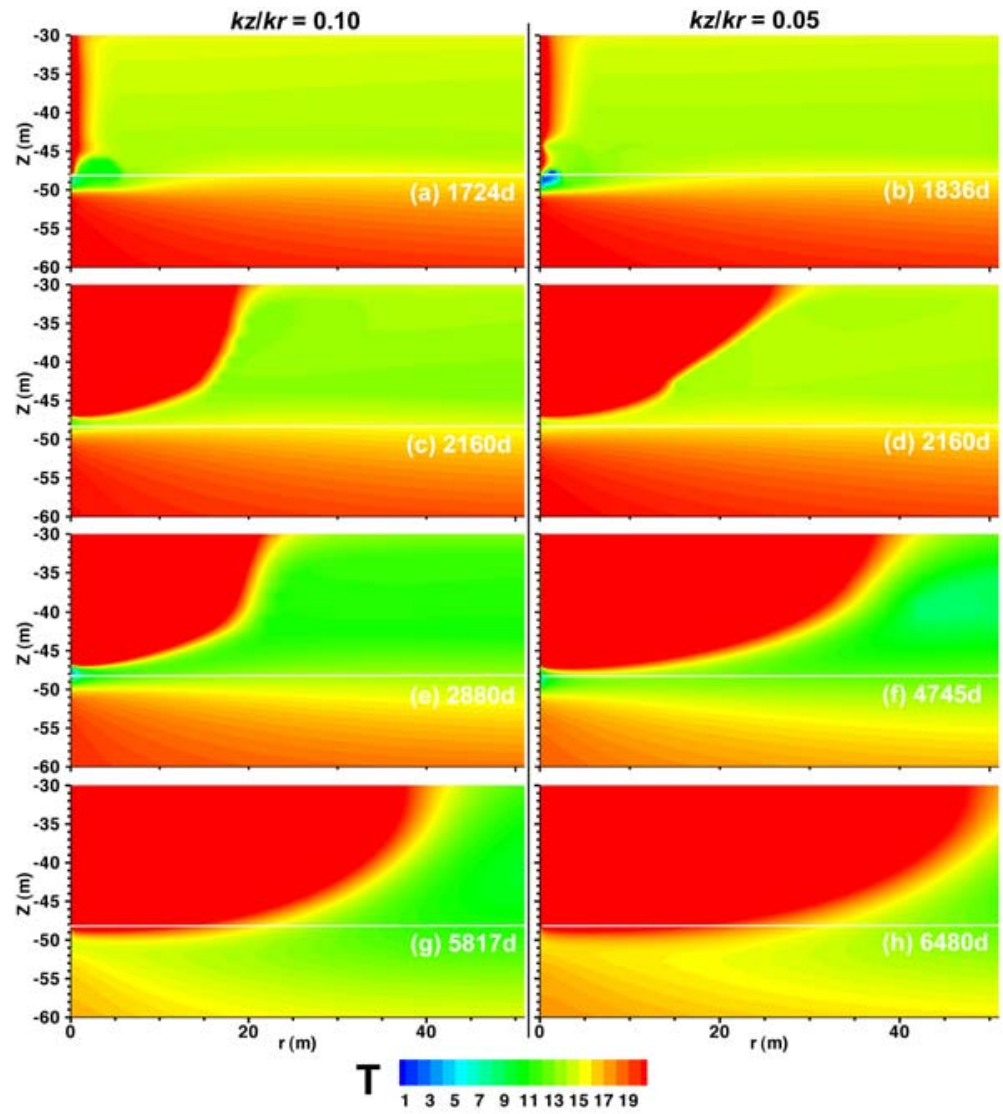

Figure 16 - Evolution of the spatial distribution of $T$ during gas production from the Class 2 oceanic hydrate deposit with varying anisotropy. 


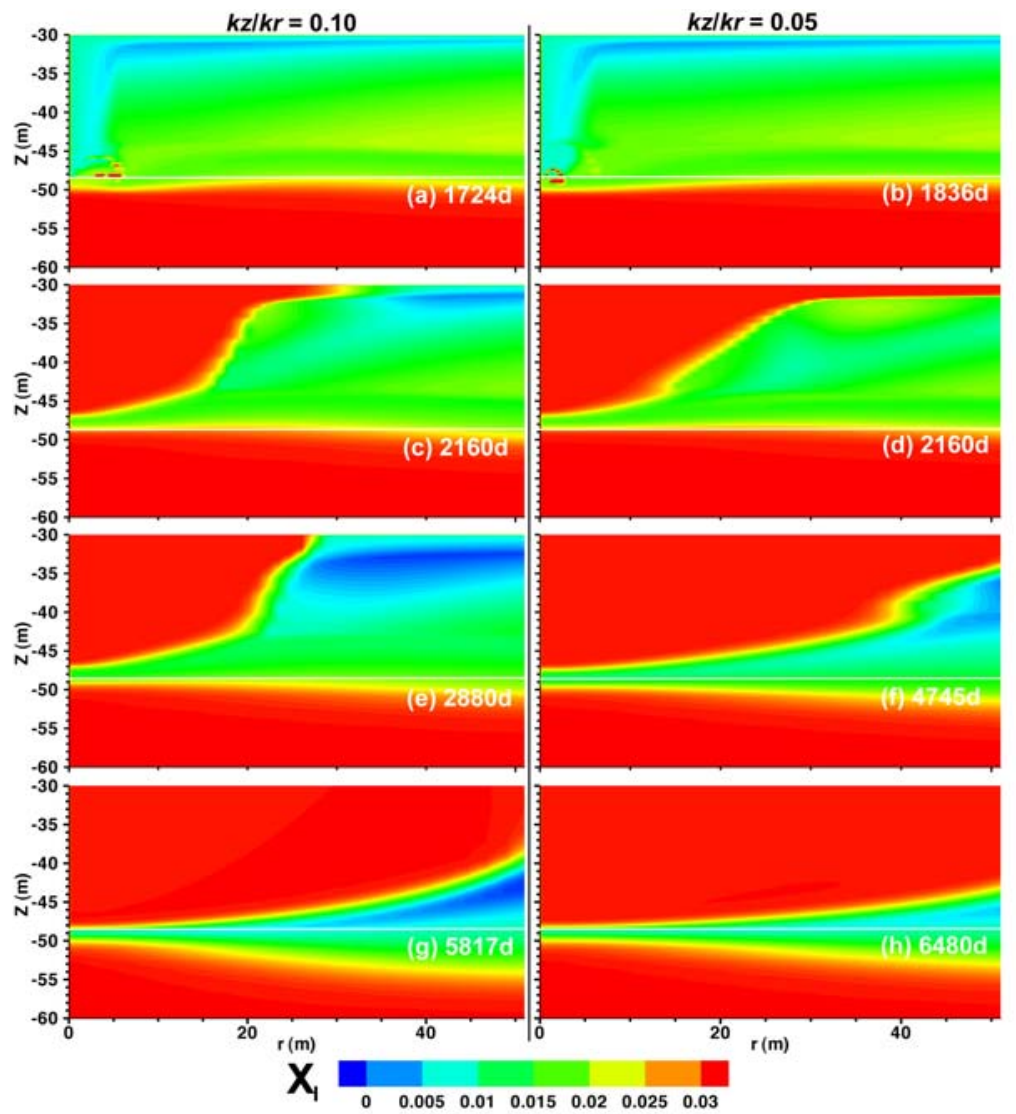

Figure 17 - Evolution of the spatial distribution of $X_{l}$ during gas production from the Class 2 oceanic hydrate deposit with varying anisotropy.

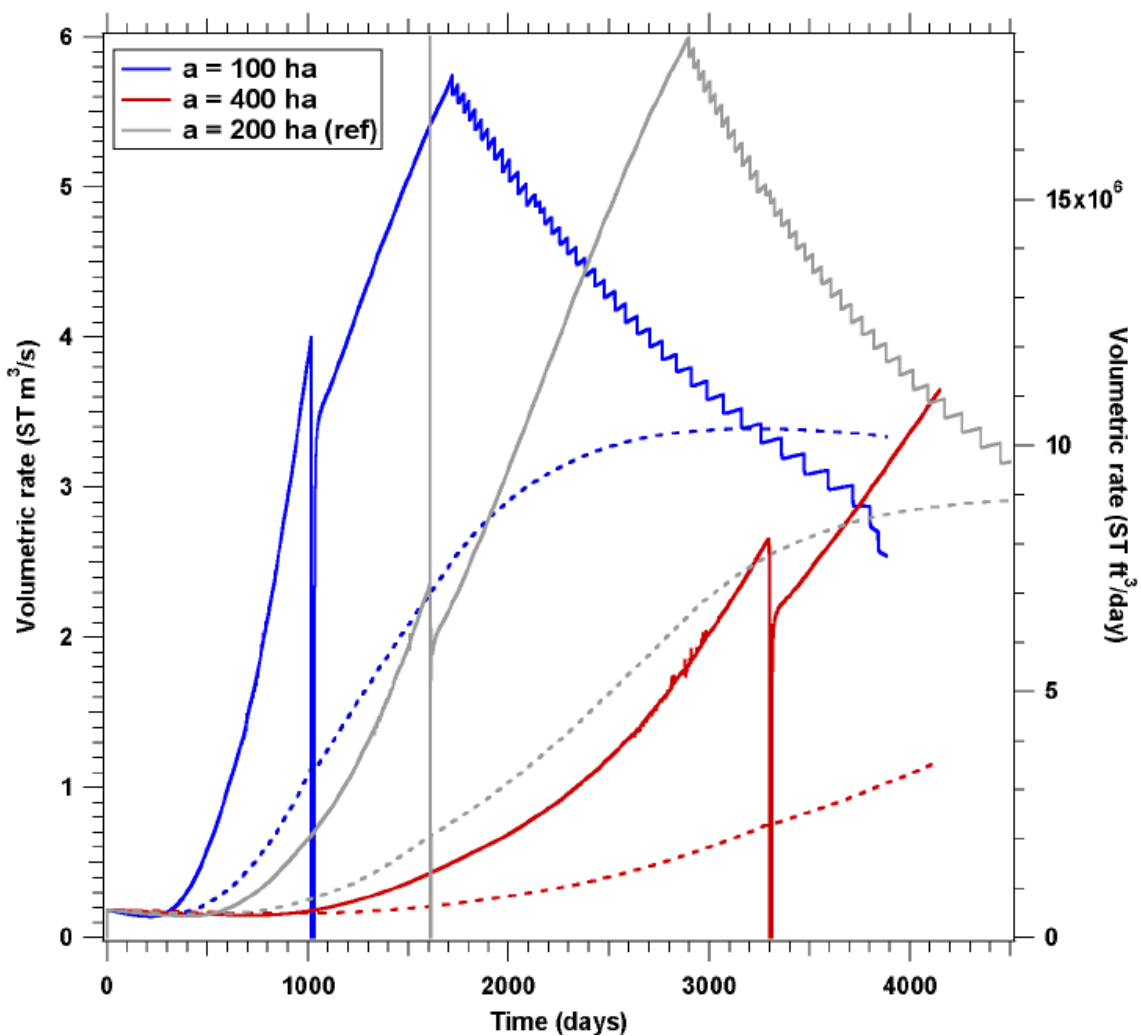

Figure 18 - Rate of $\mathrm{CH}_{4}$ production at the well, $Q_{P}$ (solid lines), and the evolution of average production (dotted lines) during production from a Class 2 oceanic deposit with varying well spacing. 


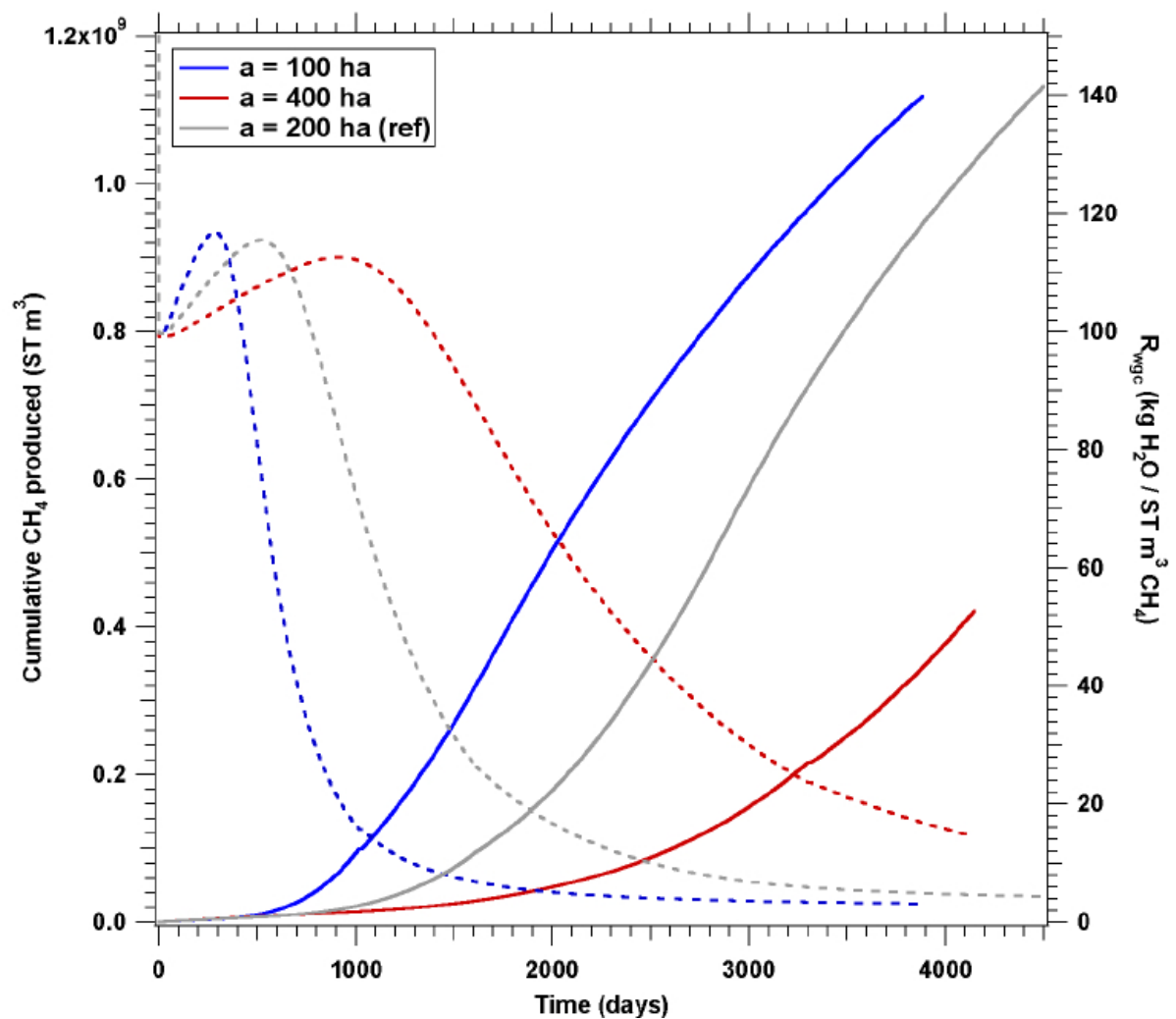

Figure 19 - Cumulative volume of $\mathrm{CH}_{4}$ produced at the well, $V_{P}$ (solid lines), and the cumulative water-to-gas ratio, $R_{W G C}$ (dotted lines), during production from a Class 2 oceanic deposit with varying well spacing.

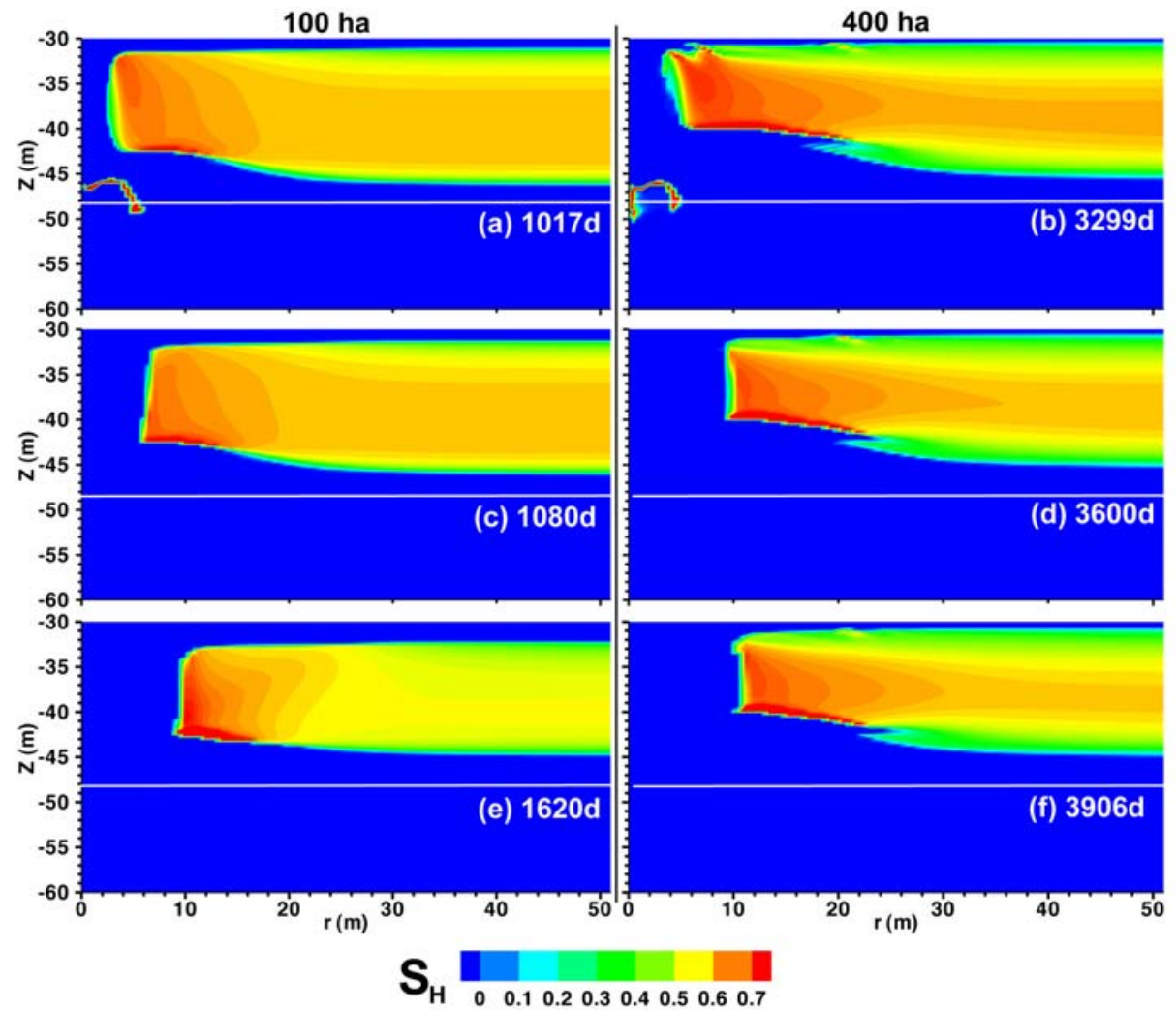

Figure 20 - Evolution of the spatial distribution of $S_{H}$ during gas production from the Class 2 oceanic hydrate deposit with varying well spacing. 


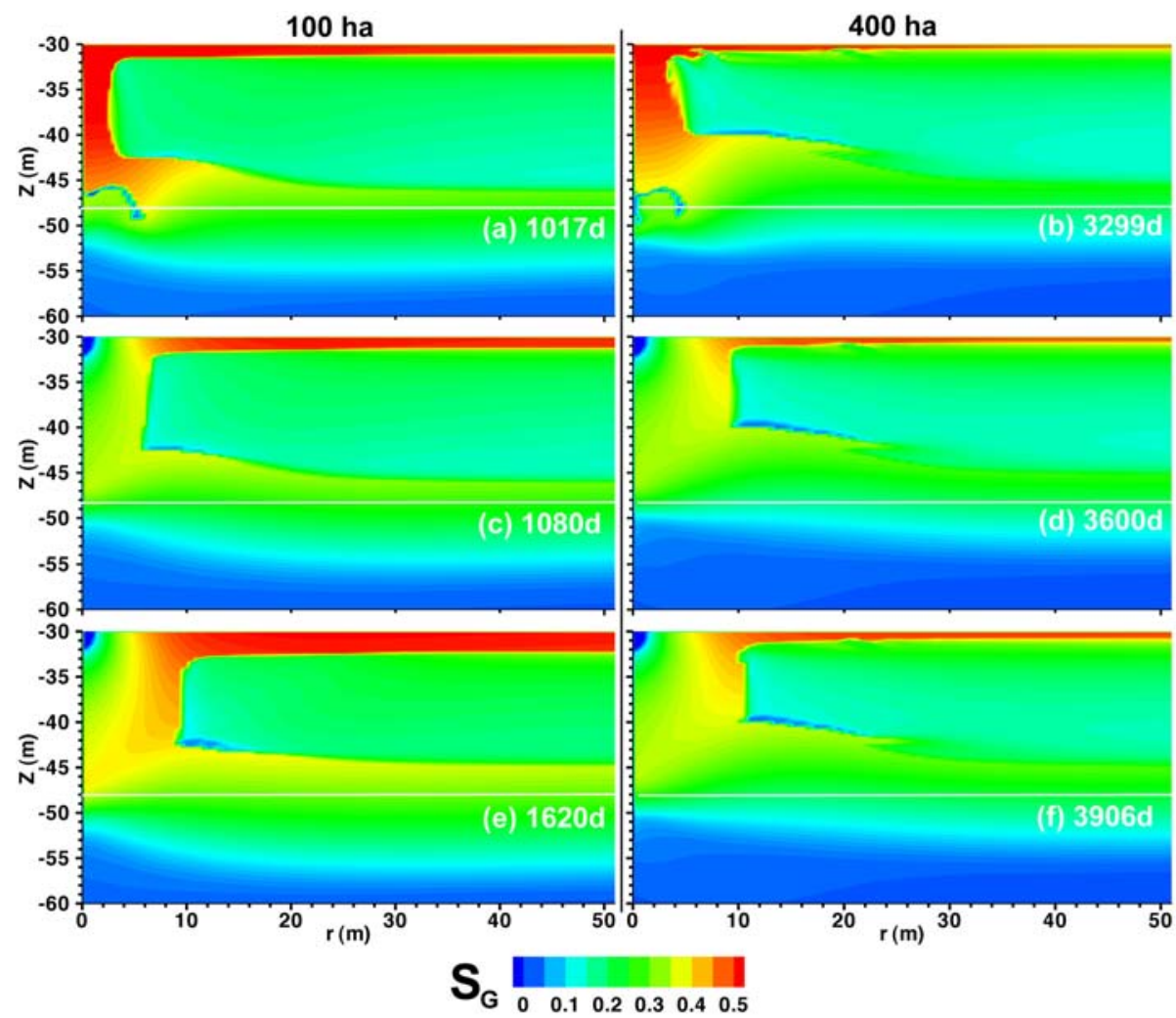

Figure 21 - Evolution of the spatial distribution of $S_{G}$ during gas production from the Class 2 oceanic hydrate deposit with varying well spacing.

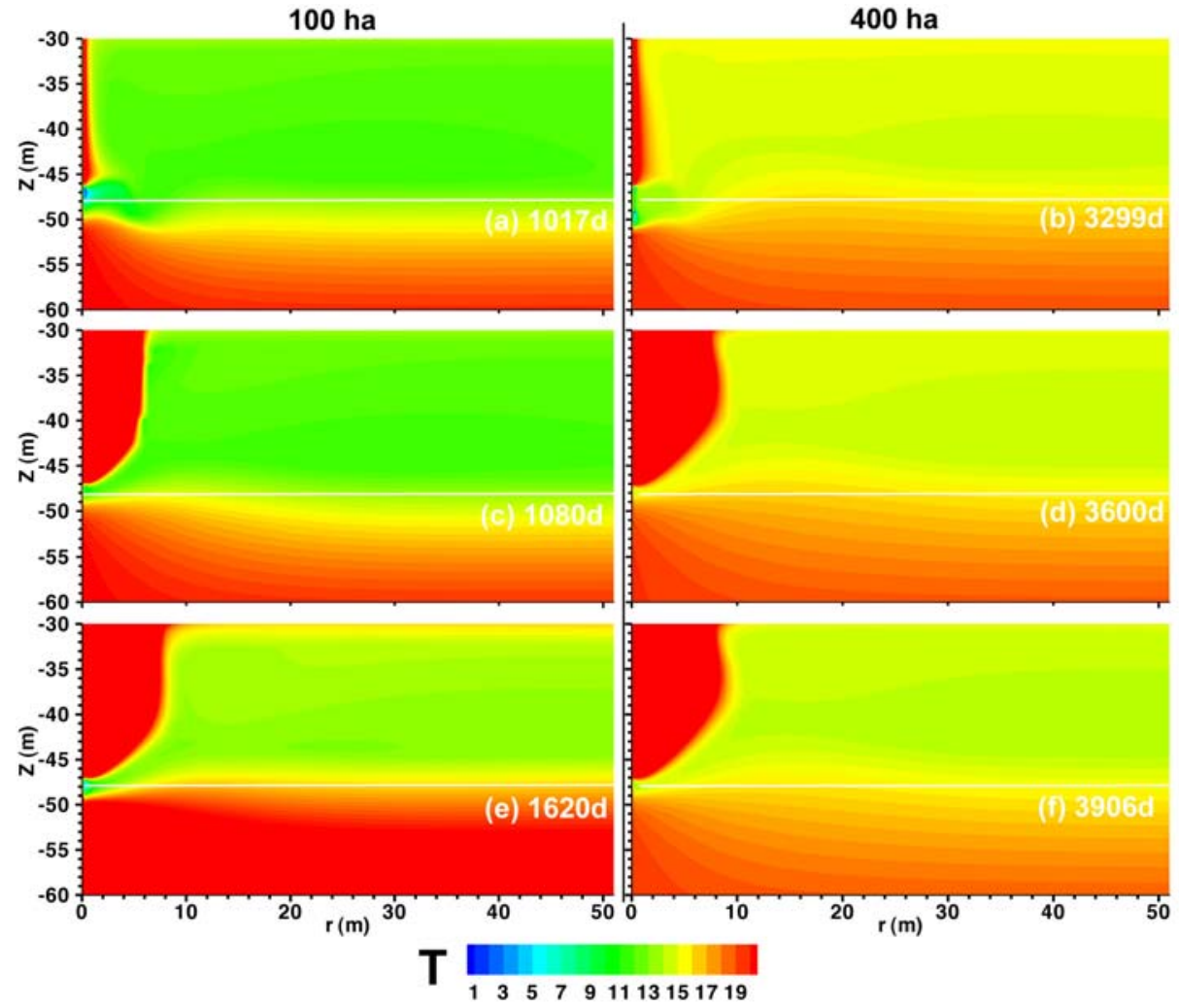

Figure 22 - Evolution of the spatial distribution of $T$ during gas production from the Class 2 oceanic hydrate deposit with varying well spacing. 


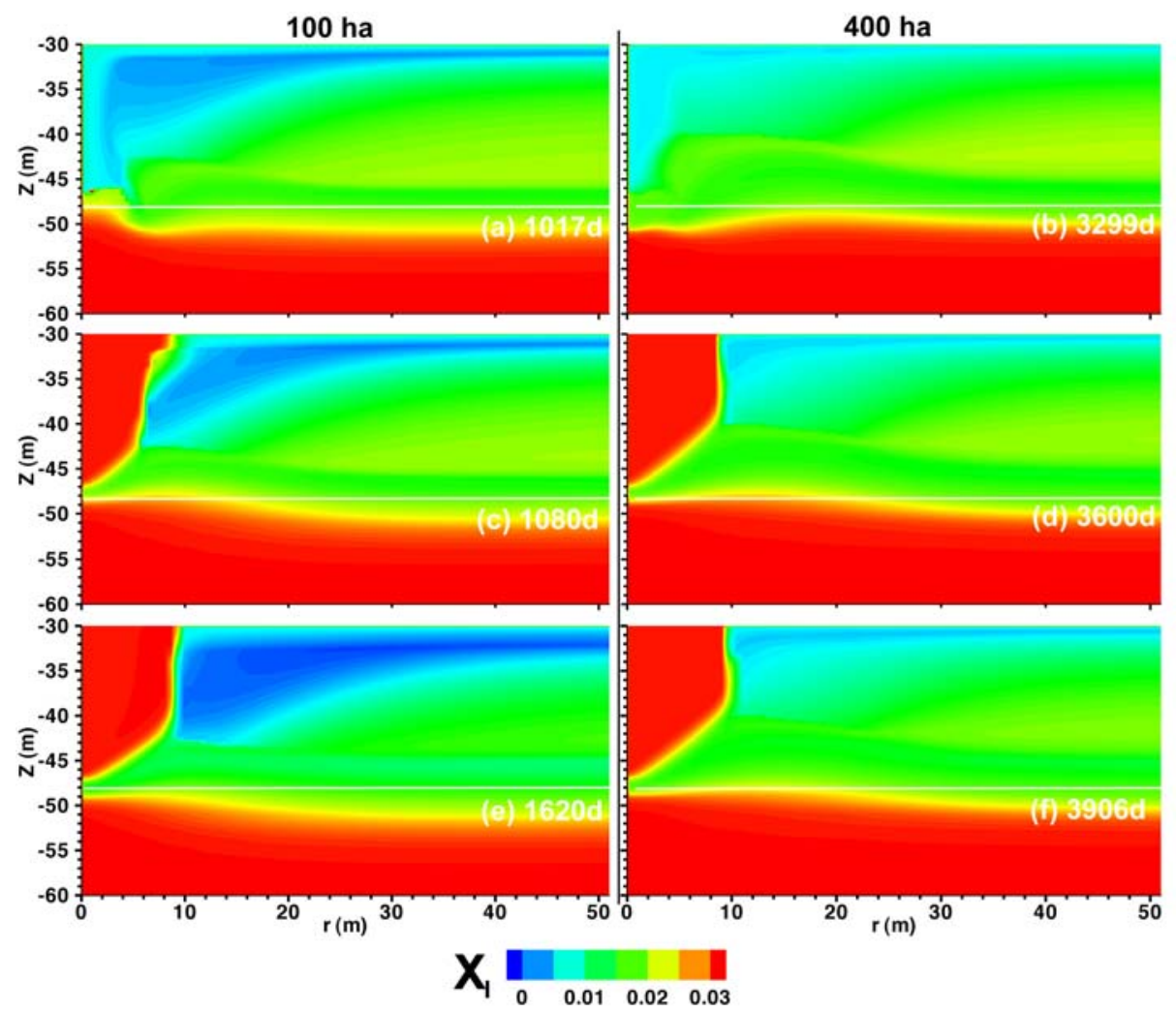

Figure 23 - Evolution of the spatial distribution of $X_{l}$ during gas production from the Class 2 oceanic hydrate deposit with varying well spacing.

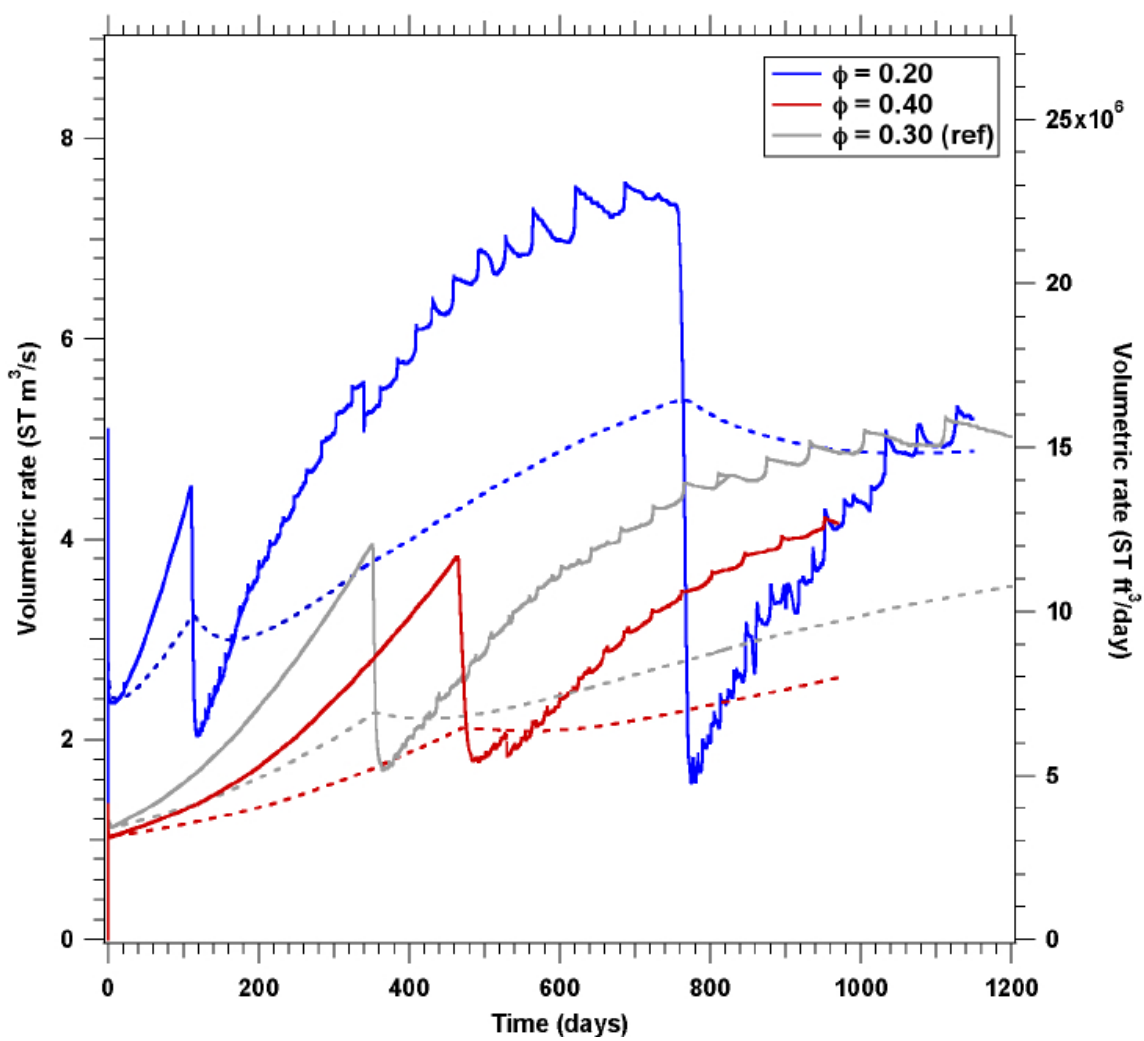

Figure 24 - Rate of $\mathrm{CH}_{4}$ production at the well, $Q_{P}$ (solid lines), and the evolution of average production (dotted lines) during production from a Class 3 oceanic deposit at varying porosity. 


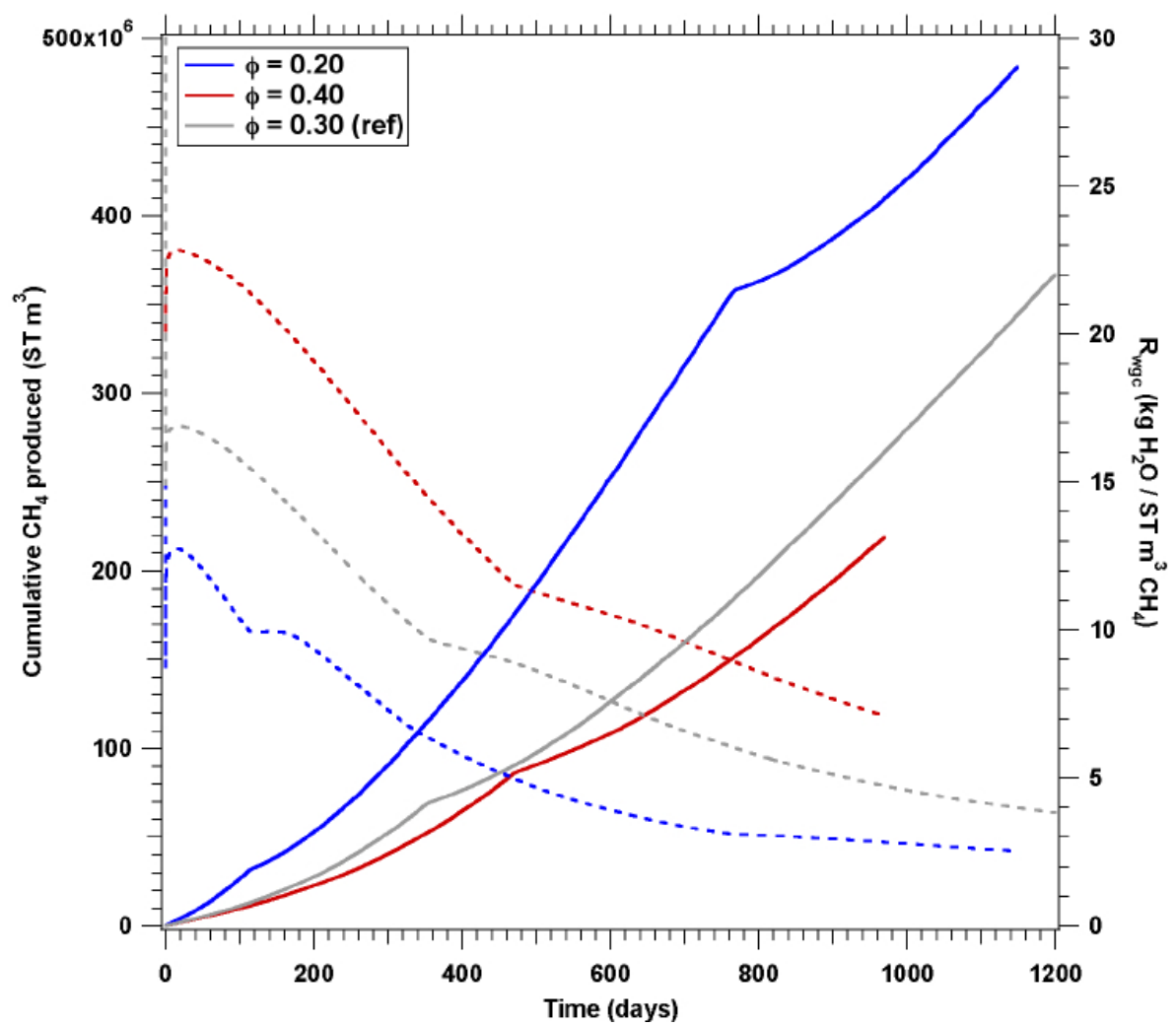

Figure 25 - Cumulative volume of $\mathrm{CH}_{4}$ produced at the well, $V_{P}$ (solid lines), and the cumulative water-to-gas ratio, $\boldsymbol{R}_{W G C}$ (dotted lines), during production from a Class 3 oceanic deposit at varying porosity.
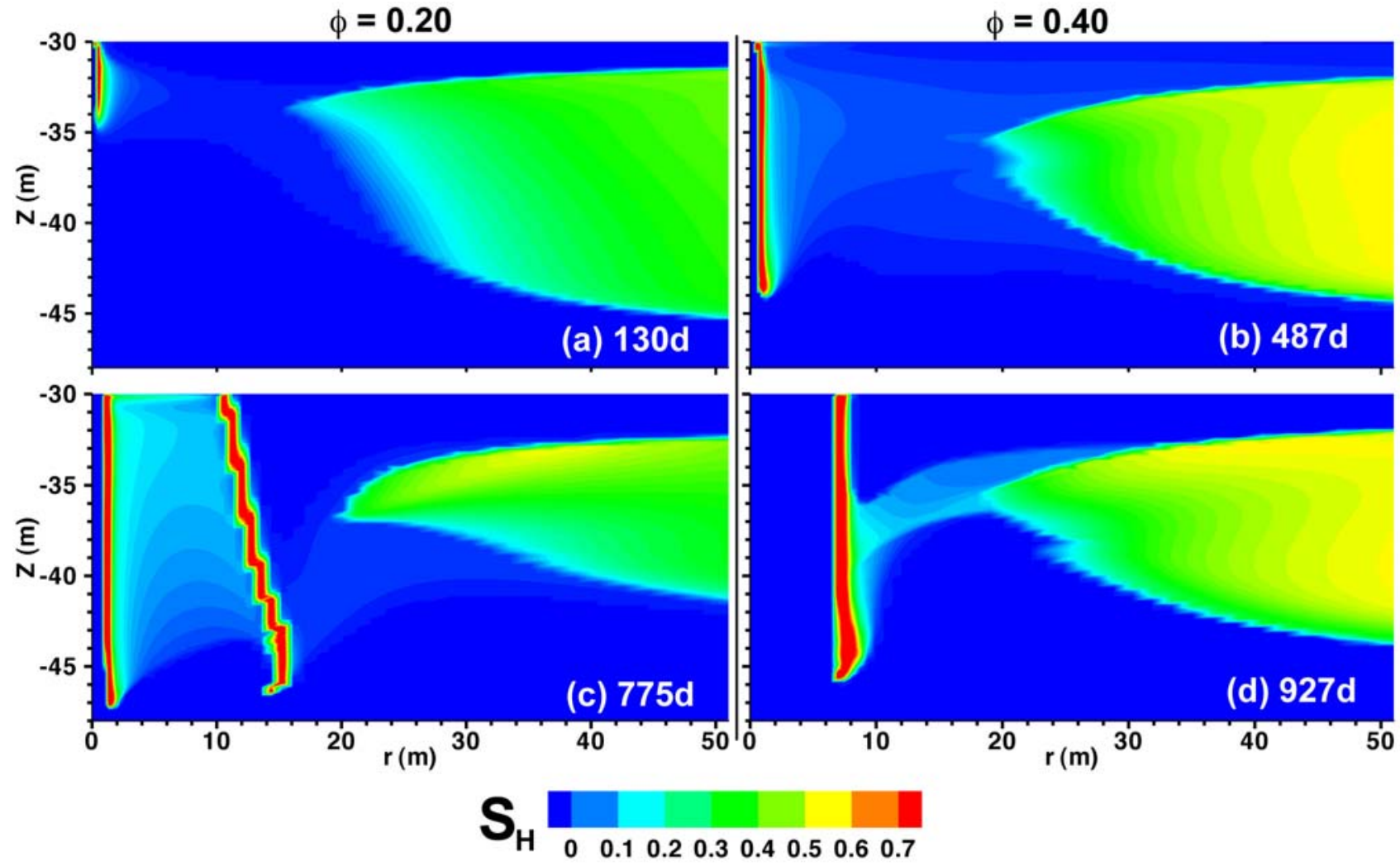

Figure 26 - Evolution of the spatial distribution of $S_{H}$ during gas production from the Class 3 oceanic hydrate deposit with varying porosity. 


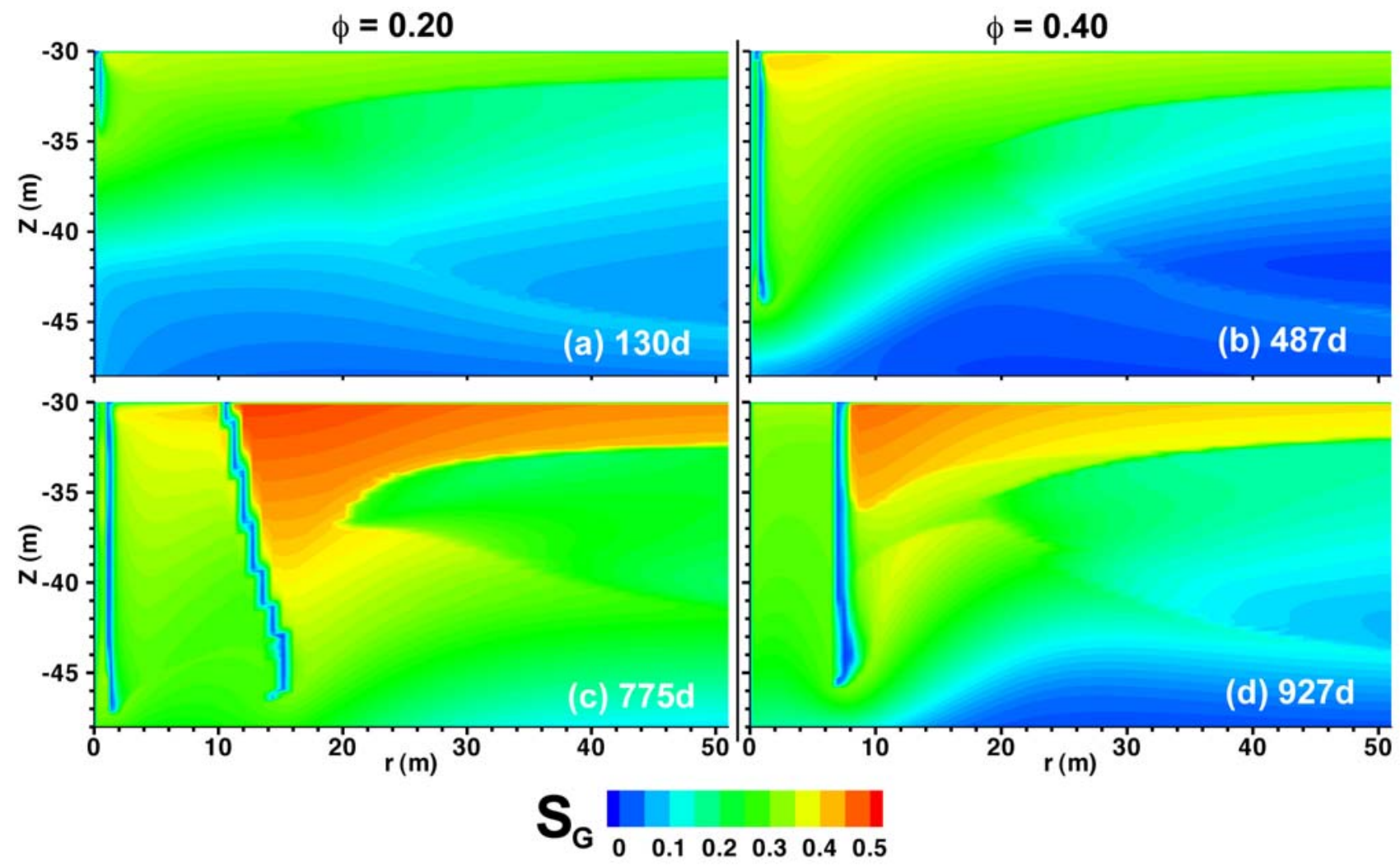

Figure 27 - Evolution of the spatial distribution of $S_{G}$ during gas production from the Class 3 oceanic hydrate deposit with varying porosity.

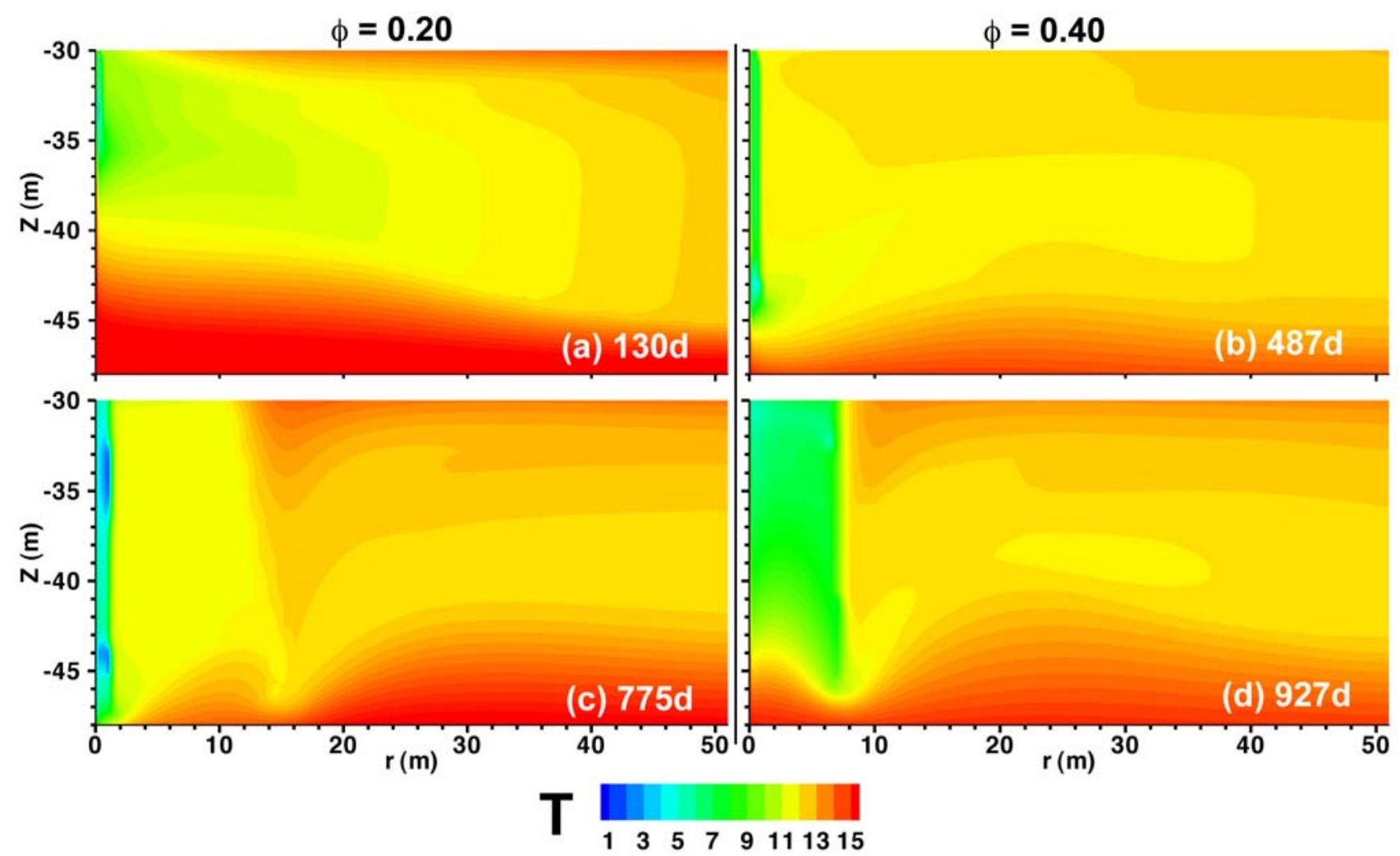

Figure 28 - Evolution of the spatial distribution of $T$ during gas production from the Class 3 oceanic hydrate deposit with varying porosity. 


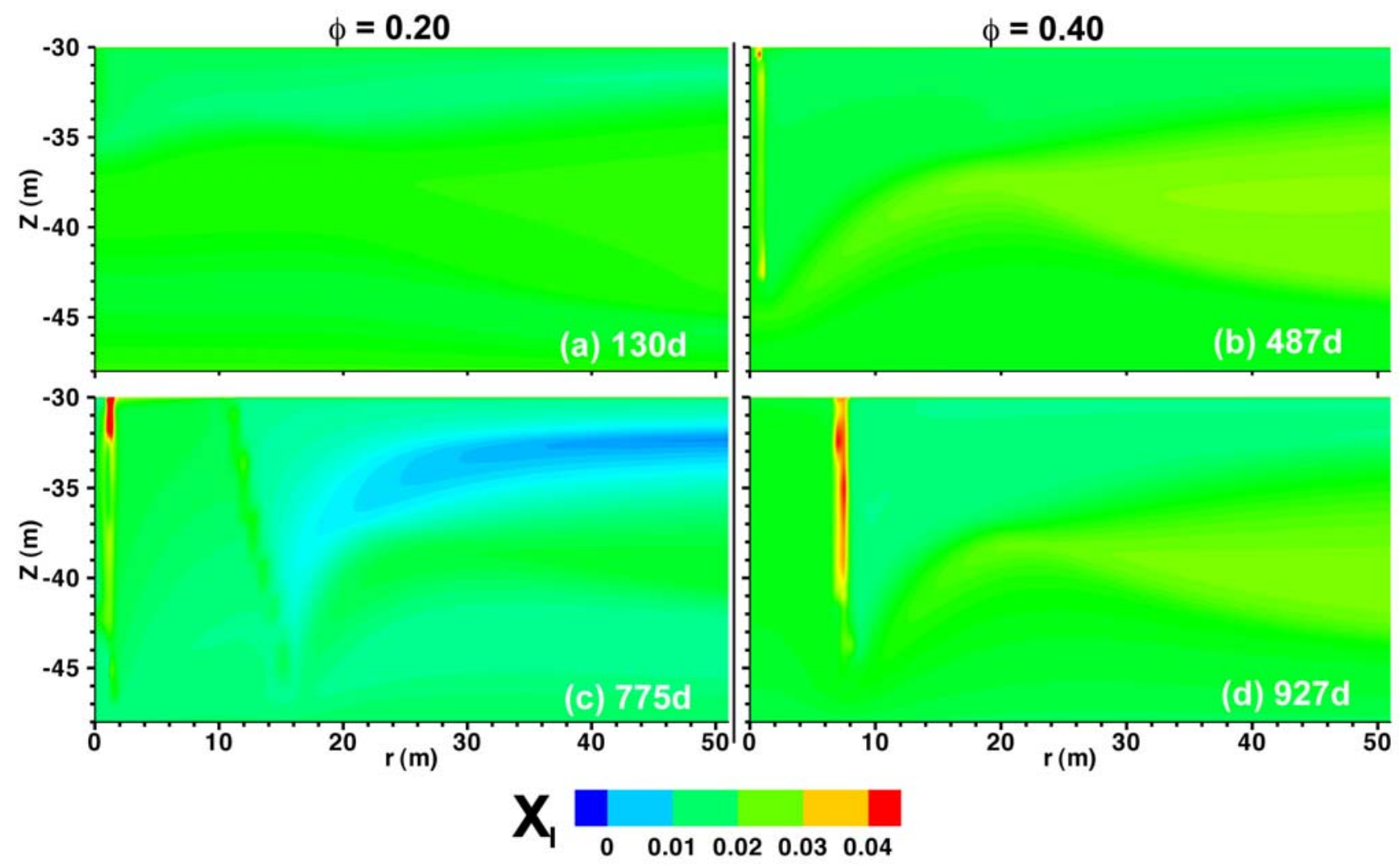

Figure 29 - Evolution of the spatial distribution of $X_{l}$ during gas production from the Class 3 oceanic hydrate deposit with varying porosity.

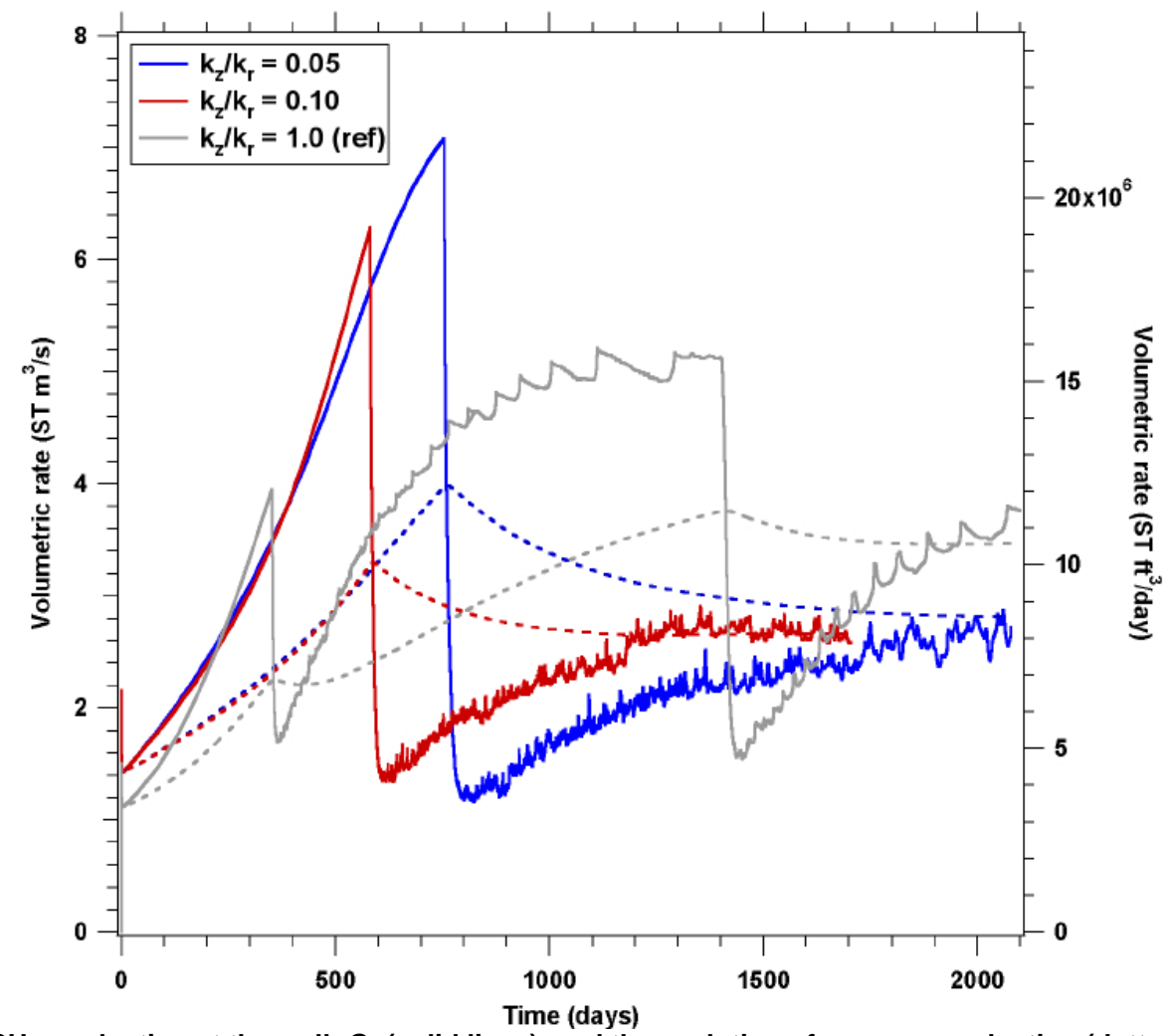

Figure 30 - Rate of $\mathrm{CH}_{4}$ production at the well, $Q_{P}$ (solid lines), and the evolution of average production (dotted lines) during production from a Class 3 oceanic deposit with varying anisotropy. 


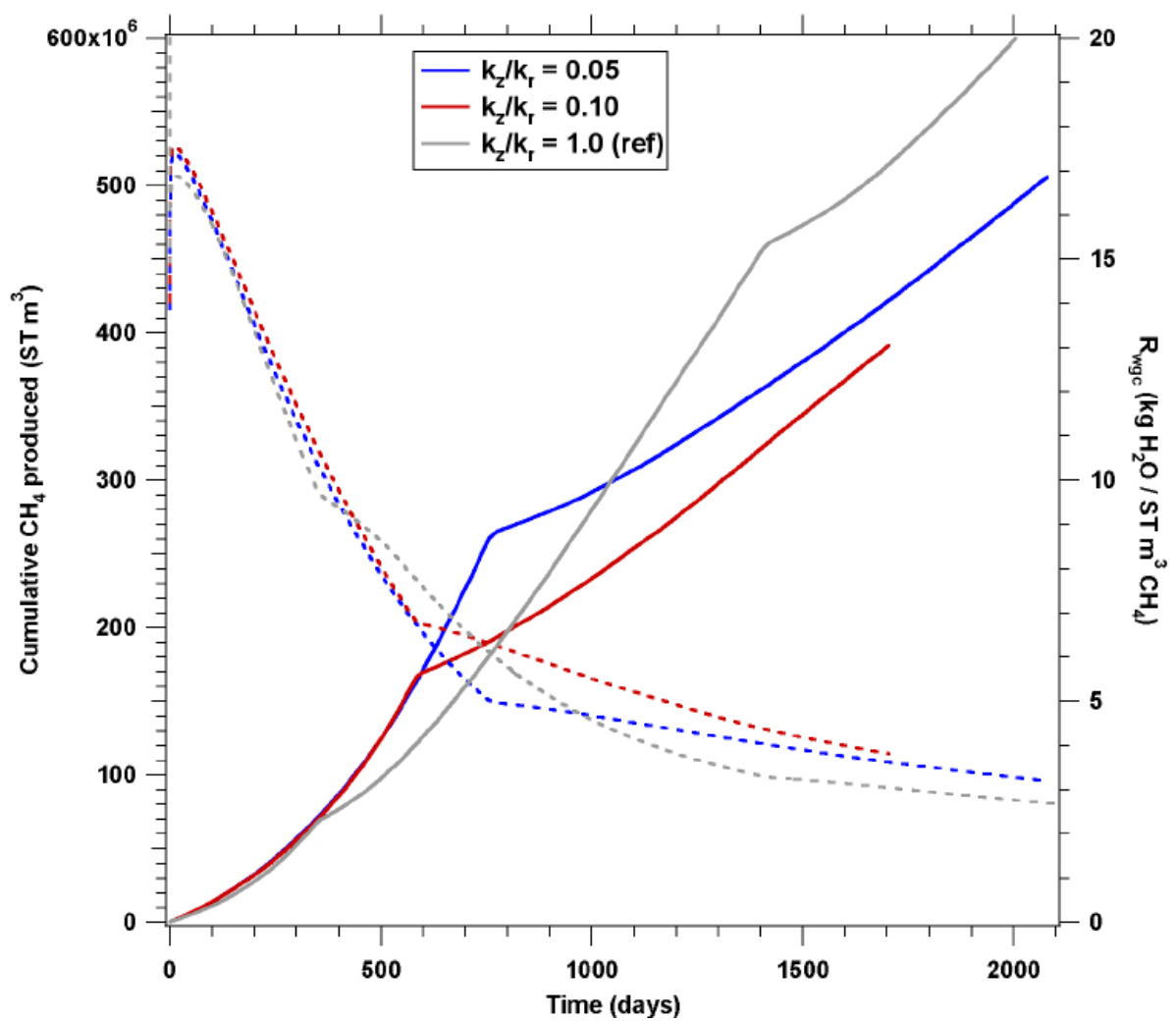

Figure 31 - Cumulative volume of $\mathrm{CH}_{4}$ produced at the well, $\boldsymbol{V}_{P}$ (solid lines), and the cumulative water-to-gas ratio, $\boldsymbol{R}_{W G C}$ (dotted lines), during production from a Class 3 oceanic deposit with varying anisotropy.

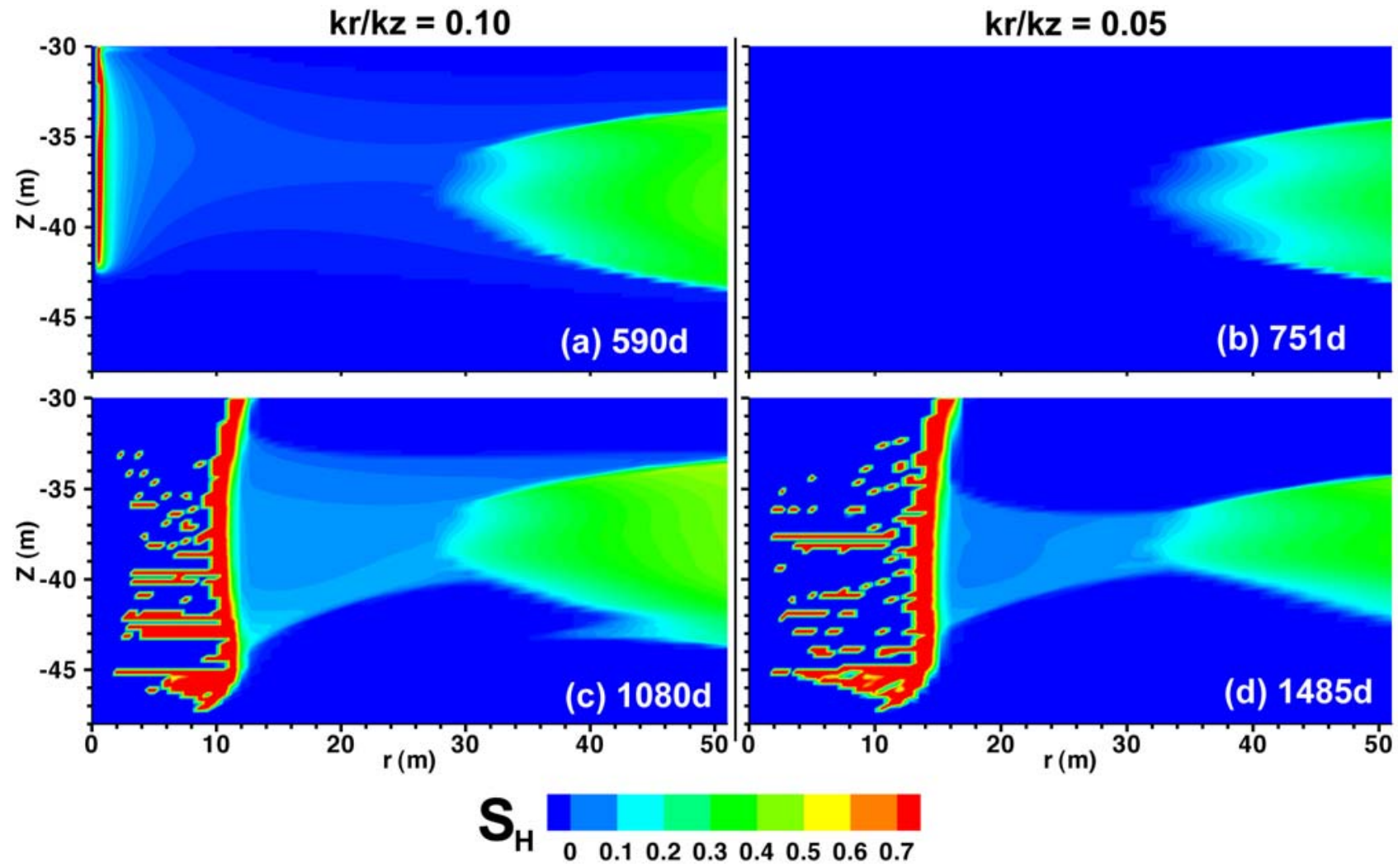

Figure 32 - Evolution of the spatial distribution of $S_{H}$ during gas production from the Class 3 oceanic hydrate deposit with varying anisotropy. 


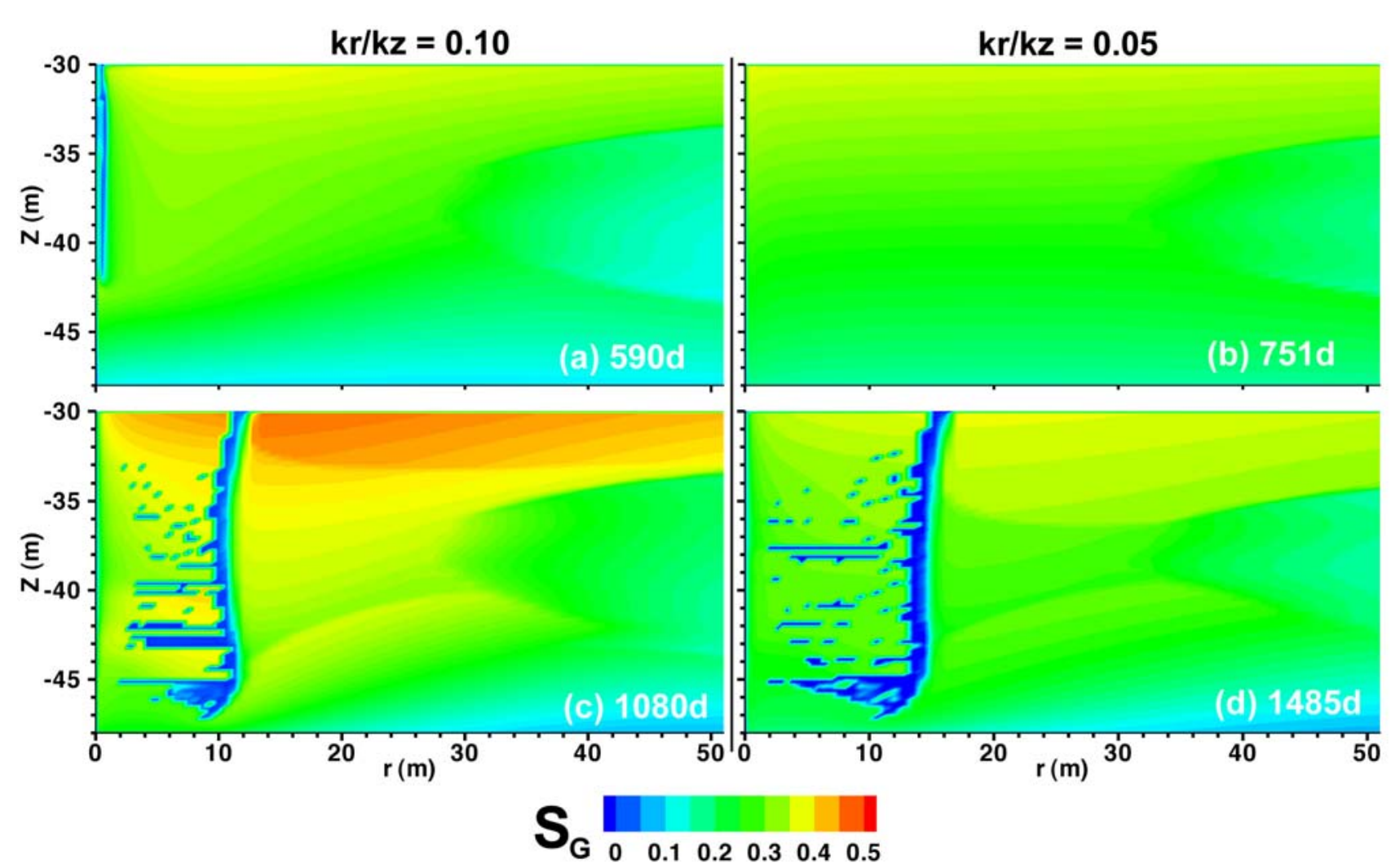

Figure 33 - Evolution of the spatial distribution of $S_{G}$ during gas production from the Class 3 oceanic hydrate deposit with varying anisotropy.

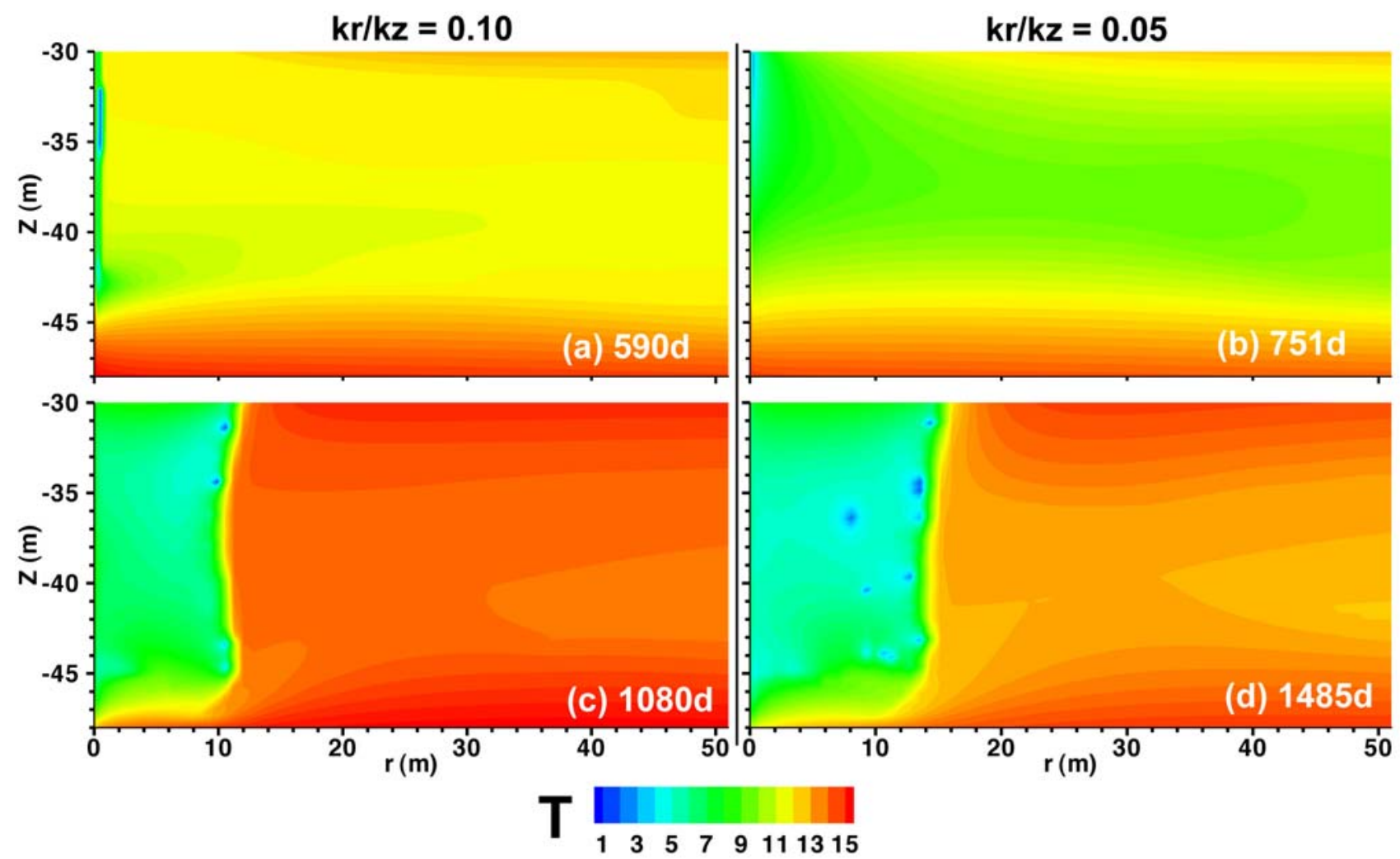

Figure 34 - Evolution of the spatial distribution of $T$ during gas production from the Class 3 oceanic hydrate deposit with varying anisotropy. 


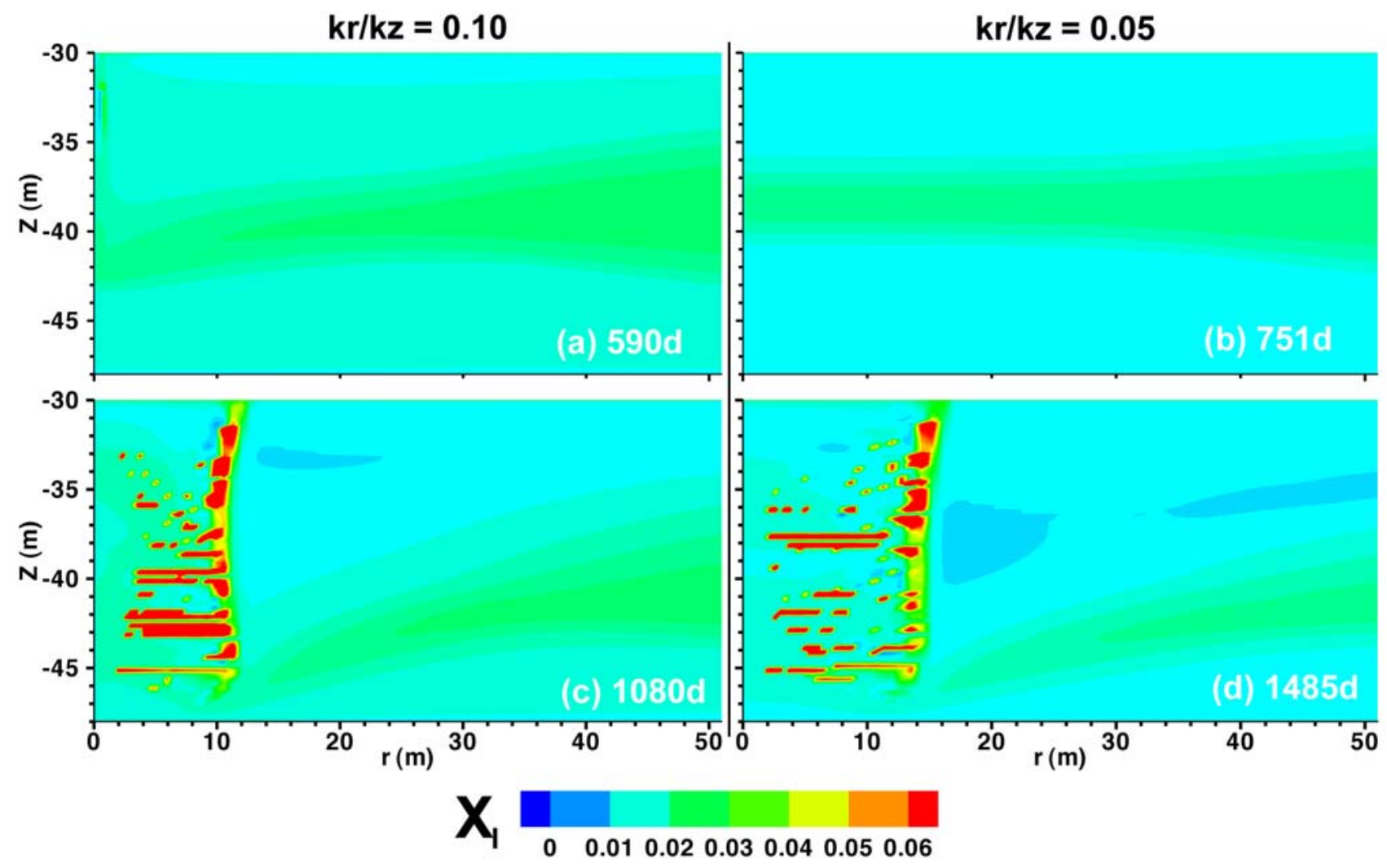

Figure 35 - Evolution of the spatial distribution of $X_{I}$ during gas production from the Class 3 oceanic hydrate deposit with varying anisotropy.

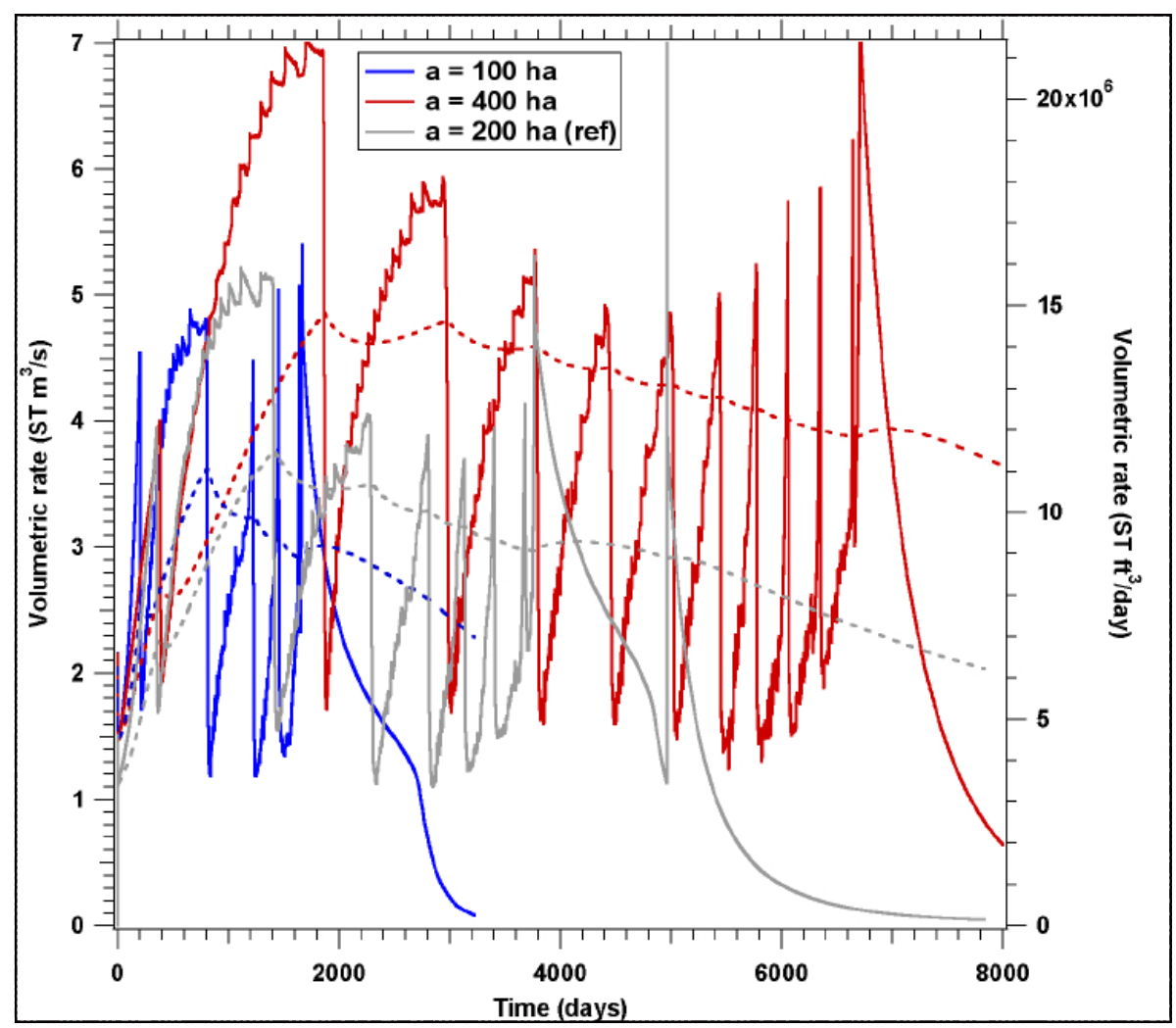

Figure 36 - Rate of $\mathrm{CH}_{4}$ production at the well, $Q_{P}$ (solid lines), and the evolution of average production (dotted lines) during production from a Class 3 oceanic deposit with varying well spacing. 


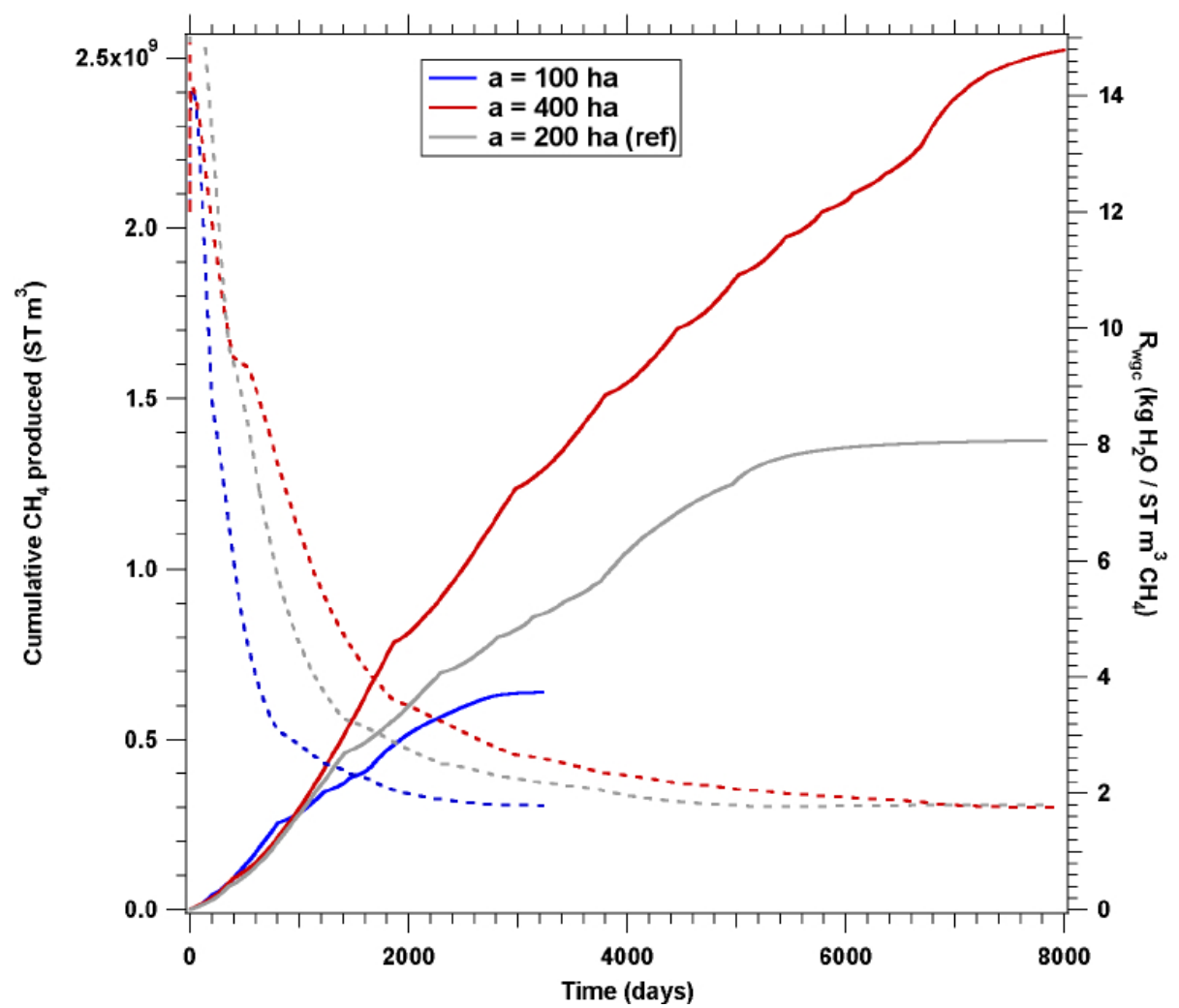

Figure 37 - Cumulative volume of $\mathrm{CH}_{4}$ produced at the well, $V_{P}$ (solid lines), and the cumulative water-to-gas ratio, $\boldsymbol{R}_{W G C}$ (dotted lines), during production from a Class 3 oceanic deposit with varying well spacing.

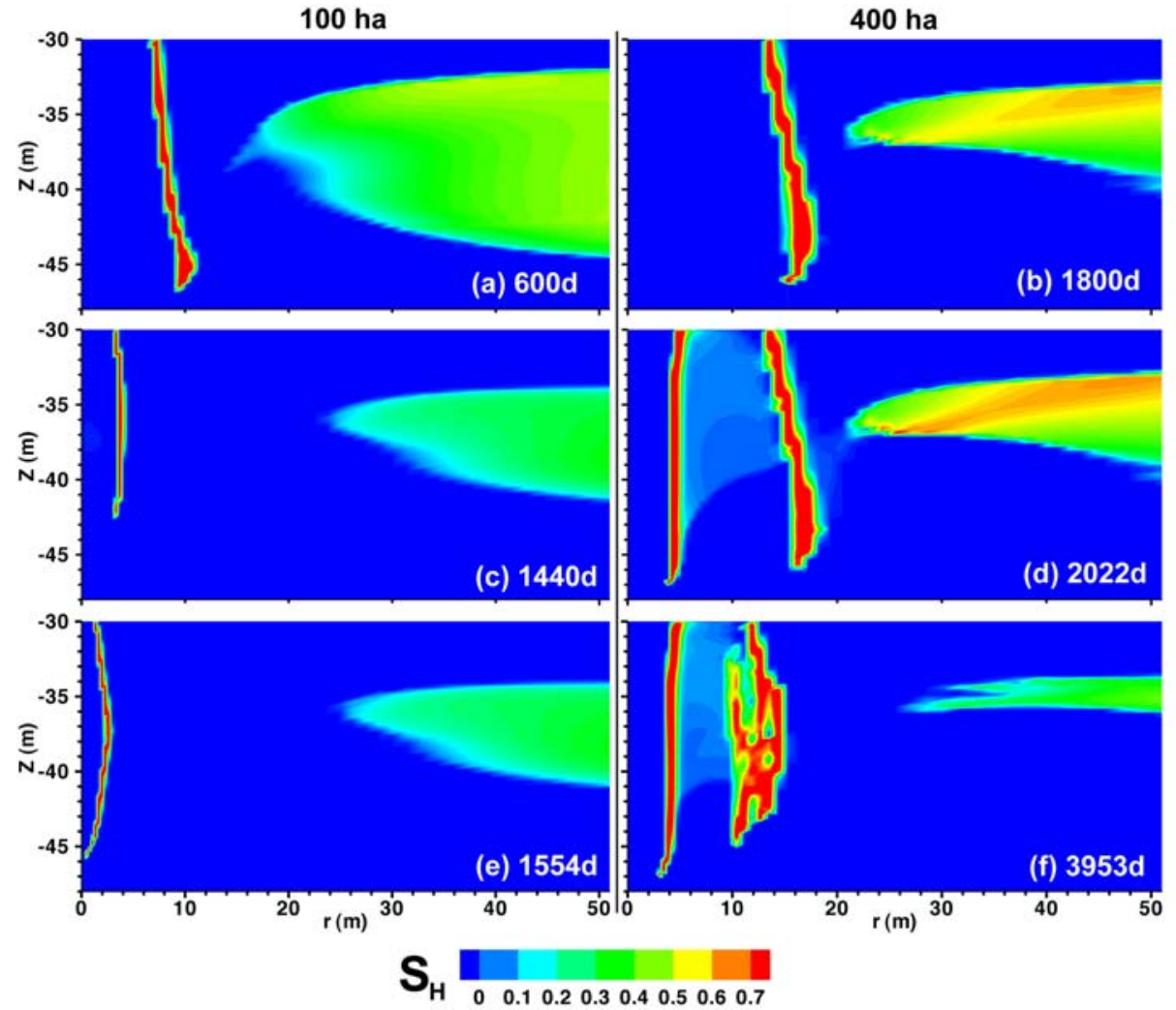

Figure 38 - Evolution of the spatial distribution of $S_{H}$ during gas production from the Class 3 oceanic hydrate deposit with varying well spacing. 


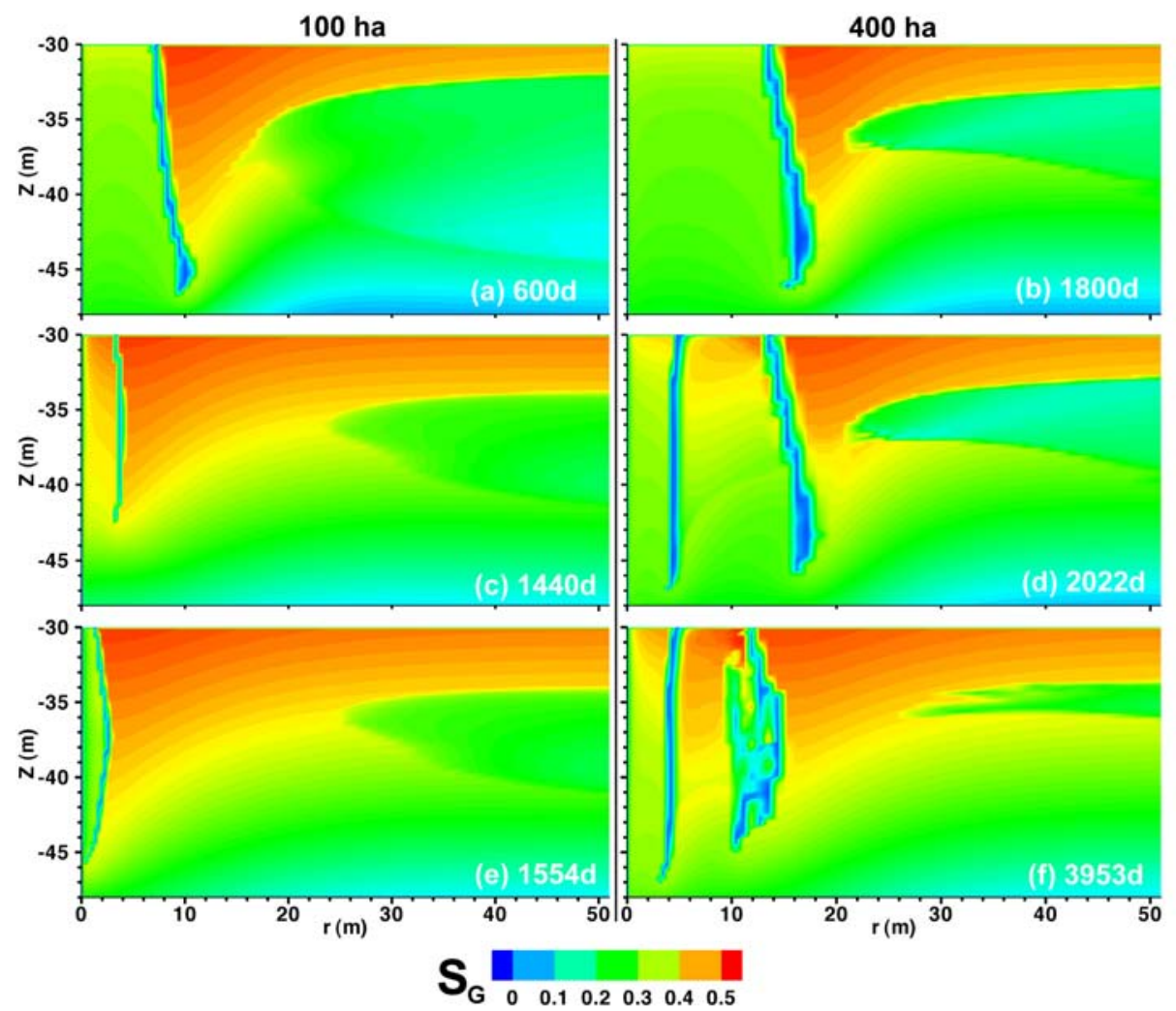

Figure 39 - Evolution of the spatial distribution of $S_{G}$ during gas production from the Class 3 oceanic hydrate deposit with varying well spacing.

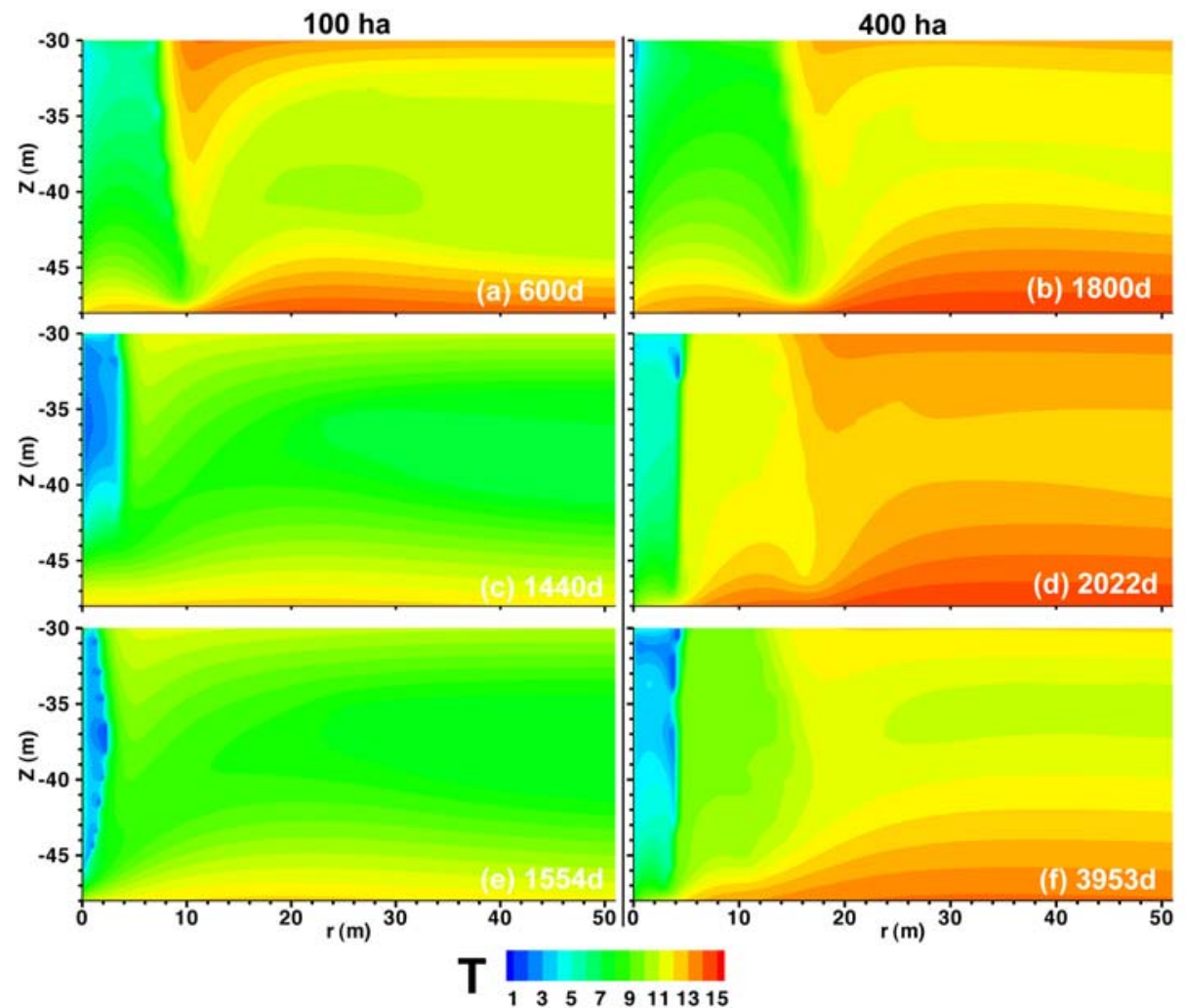

Figure 40 - Evolution of the spatial distribution of $T$ during gas production from the Class 3 oceanic hydrate deposit with varying well spacing. 


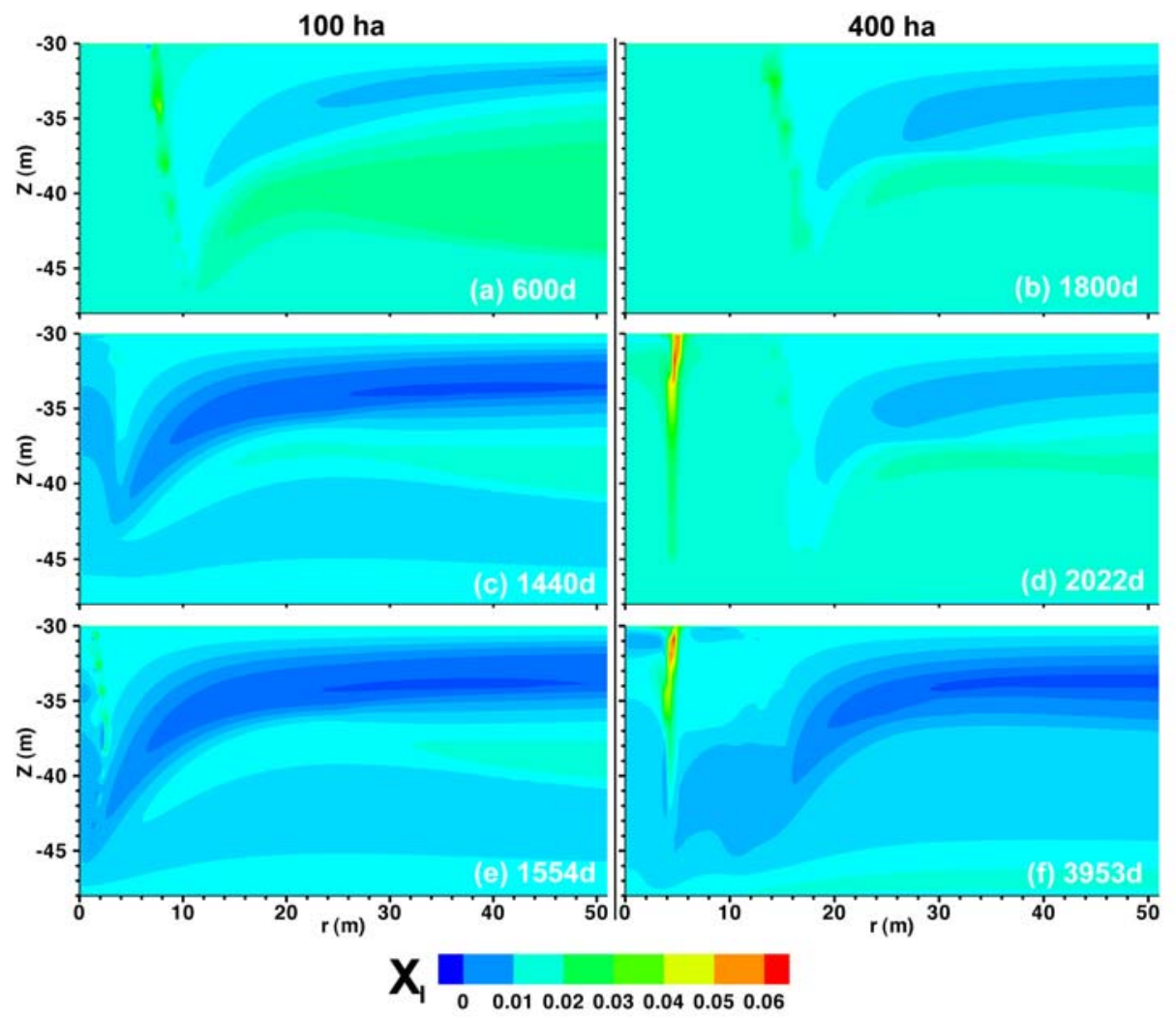

Figure 41 - Evolution of the spatial distribution of $X_{l}$ during gas production from the Class 3 oceanic hydrate deposit with varying well spacing.

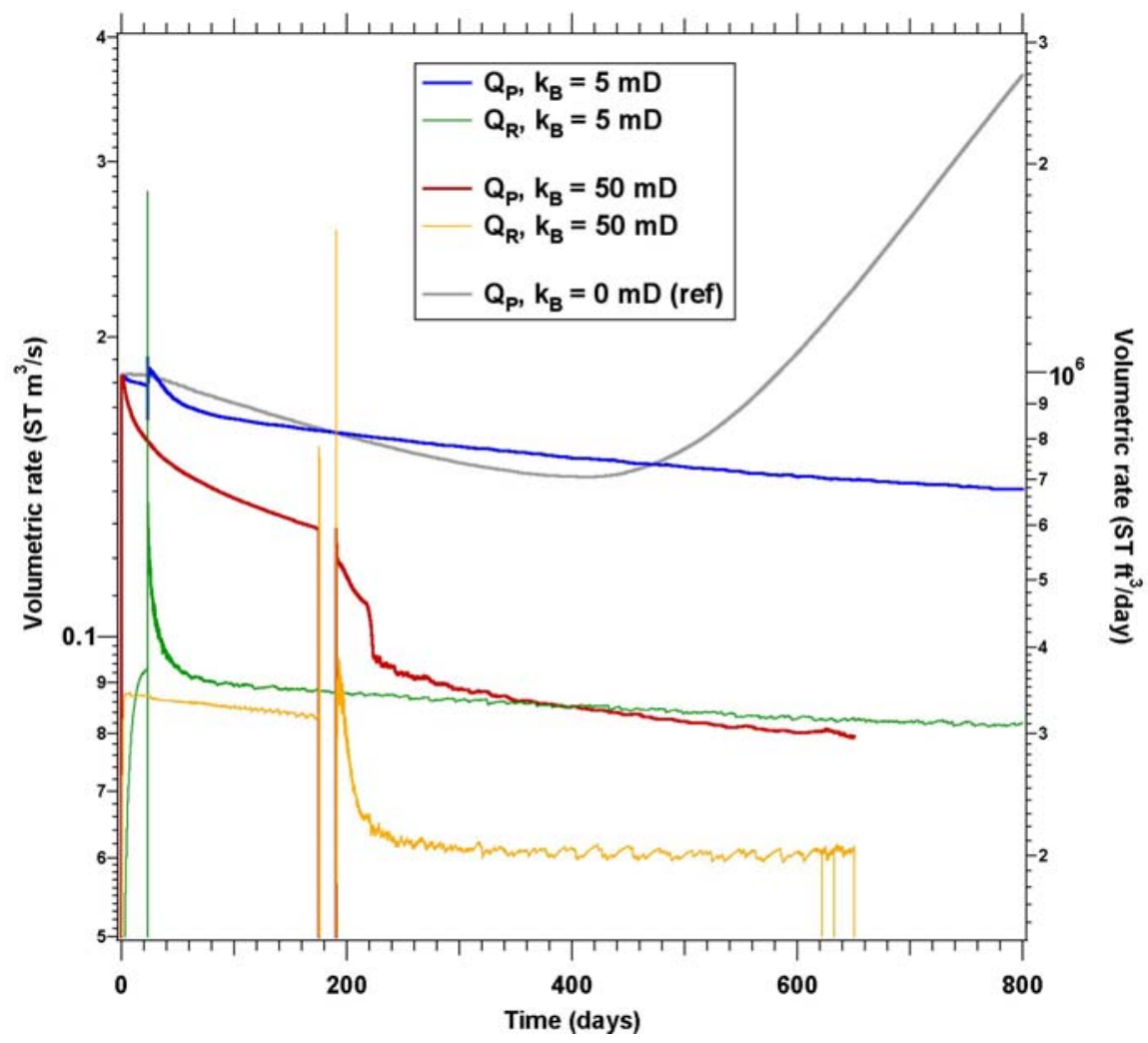

Figure 42 - Rates of $\mathrm{CH}_{4}$ production at the well, $Q_{P}$, and the rate of $\mathrm{CH} 4$ release from hydrate dissociation, $Q_{R}$, during production from a Class 2 oceanic deposit with permeable boundaries. 


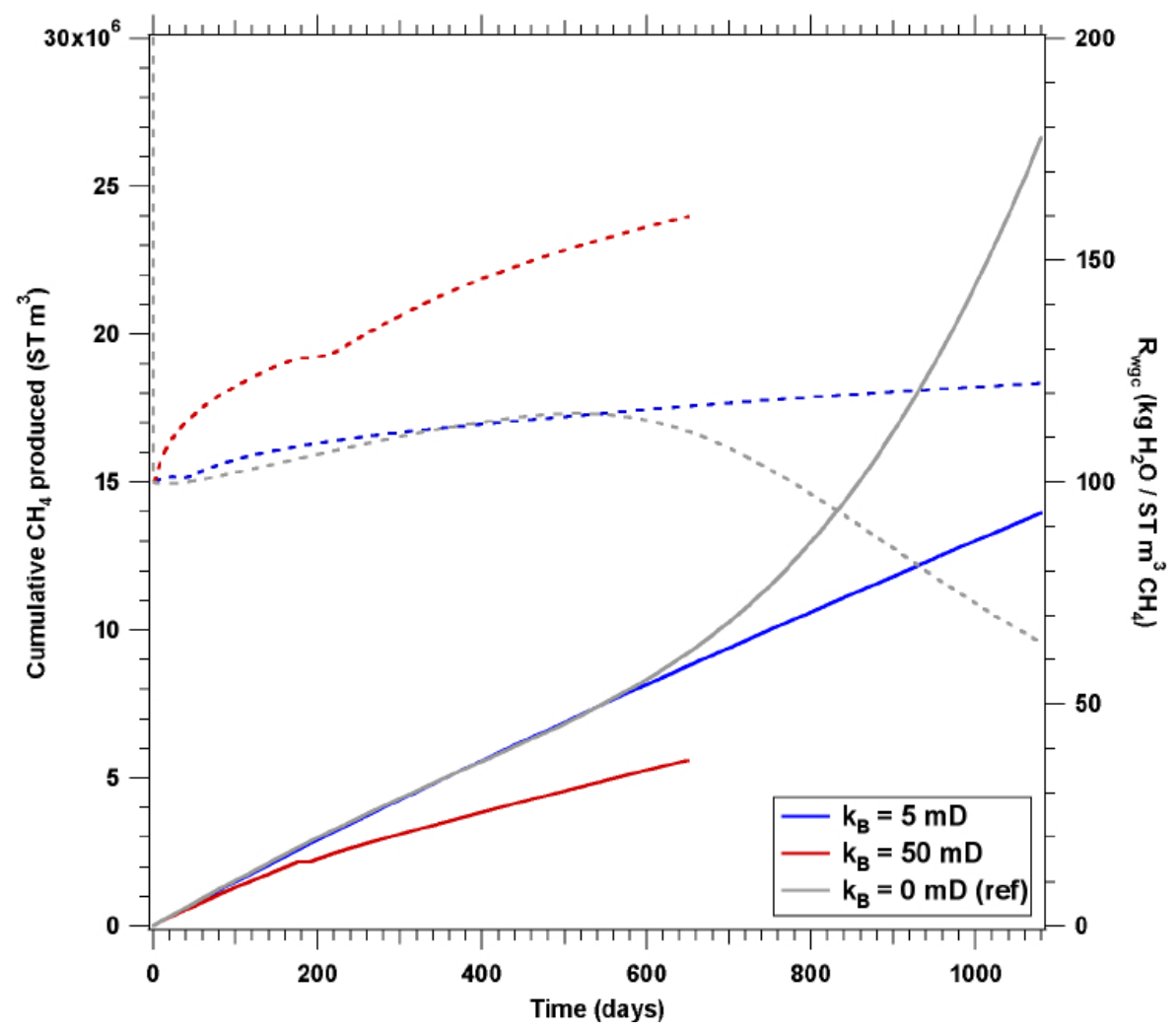

Figure 43 - Cumulative volume of $\mathrm{CH}_{4}$ produced at the well, $V_{P}$ (solid lines), and the cumulative water-to-gas ratio, $\boldsymbol{R}_{W G C}$ (dotted lines), during production from a Class 2 oceanic deposit with permeable boundaries. 